Florida International University

FIU Digital Commons

\title{
The Relationship Between Spiritualilty, Knowledge and Tuberculosis (TB) Medication Adherence Among African Americans And Haitians.
}

Regina Y. McDade

Florida International University, regcarp1@yahoo.com

DOI: $10.25148 /$ etd.FI10080902

Follow this and additional works at: https://digitalcommons.fiu.edu/etd

Part of the Other Education Commons

\section{Recommended Citation}

McDade, Regina Y., "The Relationship Between Spiritualilty, Knowledge and Tuberculosis (TB) Medication Adherence Among African Americans And Haitians." (2010). FIU Electronic Theses and Dissertations. 267.

https://digitalcommons.fiu.edu/etd/267 


\title{
FLORIDA INTERNATIONAL UNIVERSITY \\ Miami, Florida
}

\section{THE RELATIONSHIP BETWEEN SPIRITUALITY, KNOWLEDGE AND TUBERCULOSIS (TB) MEDICATION ADHERENCE AMONG AFRICAN AMERICANS AND HAITIANS}

\author{
A dissertation submitted in partial fulfillment of the \\ requirements for the degree of \\ DOCTOR OF EDUCATION \\ in
}

ADULT EDUCATION AND HUMAN RESOURCE DEVELOPMENT

by

Regina Y. McDade

2010 
To: $\quad$ Interim Dean Delia C. Garcia

College of Education

This dissertation, written by Regina Y. McDade, and entitled The Relationship between Spirituality, Knowledge and Tuberculosis (TB) Medication Adherence among African Americans and Haitians, having been approved in respect to style and intellectual content, is referred to you for judgment.

We have read this dissertation and recommend that it be approved.

H. Virginia McCoy

Ann Nevin

Thomas Reio

Tonette S. Rocco, Major Professor

Date of Defense: July 26, 2010

The dissertation of Regina Y. McDade is approved.

Interim Dean Delia C. Garcia

College of Education

Interim Dean Kevin O'Shea

University Graduate School

Florida International University, 2010 


\section{DEDICATION}

I dedicate this dissertation to my brother, Anthony B. Carpenter (1962-1984) who fought a 12 year battle with Systemic Lupus Erythematosis. To my dear friend Cynthia Peacock (1956-2009) who encouraged me to enter the Ed.D. Program along with her, who studies came to an end due to her battle with breast cancer. I know both of them will be proud as they look down from heaven and see me graduate.

Also to my parents Reverend Dr. Willie and Judy Carpenter who taught me that all things are possible if you have faith and a little perseverance. I thank them for their continual love, prayers, support and belief in me especially at times when I doubted myself throughout the entire process of writing this dissertation. To my son, Edric McDade, thank you for your love, prayers and belief in me. I love you. Remember to believe in yourself and all things are possible, if you believe in God. 


\section{ACKNOWLEDGMENTS}

I wish to thank the members of my committee: Dr. Rocco, Dr. McCoy, Dr. Nevin. and Dr. Reio for their guidance and support. Special gratitude is extended to my major professor, Dr. Rocco, thank you for accepting me after my former major professor retired. Your guidance in writing this dissertation was priceless. You showed how much you really cared by opening your home and sacrificed family time for Saturday dissertation peer group meetings and I thank you. I would like to express my sincere appreciation and thanks to dissertation committee members Dr. Nevin and Dr. Reio for their willingness to guide and work with me during this entire dissertation process. Another special thank you is extended to Dr. H. Virginia McCoy, whose public health expertise, kindness and support guided during the entire dissertation process and my public health career.

I also wish to thank faculty members in the College of Education who have been supportive over the years, Drs. Leonard Bliss, Linda Bliss and Isadore Newman. Special thanks are extended to Caprila Alemida, Office Manager, College of Education, Office of Graduate Studies and Maria Tester, Office Manager, College of Education, Research and Grants for their professional assistance over the years.

I also wish to thank my peer review group the nurses and the dissertation group members for their support. Special thanks to Dr. Debra Pane for her valuable assistance. To Dr. Alexis Powell, Joel JeanBaptiste, M.S.W., the healthcare professionals at the county health department and county hospital, their assistance was very valuable, without them, this work would not have been possible. Thank you. Special thanks to all the TB patients who participated in this study for sharing your TB experience with me. I want to 
give thanks to God for the ability to complete my dissertation. Again, I wish to thank my parents Willie and Judy Carpenter for their love, support encouragement and prayers over the years. In addition, I want to thank Reverend Douglas Cook, Rose Harvey, Anita Faison, Portia James, Vanessa Hill and my extended family and friends for their support and encouragement. Finally, I thank my son Edric for his love and words of support. Now I can devote more time to help him grow and develop. 


\begin{abstract}
OF THE DISSERTATION
THE RELATIONSHIP BETWEEN SPIRITUALITY, KNOWLEDGE AND

TUBERCULOSIS (TB) MEDICATION ADHERENCE AMONG AFRICAN
\end{abstract}

AMERICANS AND HAITIANS

by

Regina Y. McDade

Florida International University, 2010

Miami, Florida

Professor Tonette S. Rocco, Major Professor

Tuberculosis (TB) is an infectious disease and nonadherence to medication can lead to new cases, multi-drug resistant TB, or potential death. Additionally, healthcare professionals and individuals with TB's knowledge of the disease and medication adherence are crucial for successful completion of medication therapy. Patient education is one of the most important aspects of care provided in healthcare settings (CDC, 1994). TB tends to disproportionally affect minority and economically disadvantaged patient populations. The purpose of this mixed method study was to explore the relationship between spirituality, knowledge, and TB medication adherence among African Americans and Haitians.

The primary research question was: What is the relationship between spirituality, knowledge and TB medication adherence among African Americans and Haitians? Quantitative data were gathered from 33 questionnaires and analyzed by two ANOVAs and four chi square analyses. The null hypothesis was not rejected; there was not a 
statistically significant relationship between spirituality and TB medication adherence ( $p$ $=.208$ ) among the study's African Americans and Haitians. Qualitative data concerning participants' knowledge of TB, gathered from 16 individual interviews further informed this analysis.

Secondary research questions examined the role of spirituality, knowledge of TB and medication adherence among African Americans and Haitians. Four common themes emerged across both groups to answer the secondary research questions. Interviews revealed the themes: (a) God is in control, (b) stigmatization of TB, (c) lack of knowledge, and (d) fear of death. The theme lack of knowledge about TB was found to contribute to stigmatization of TB patients. However, in this study stigma and lack of knowledge were related to initial denial of symptoms and delayed diagnosis, but not found to be related to TB medication adherence.

This study could help adult educators and health educators enhance their educational interventions, develop a better understanding of adult learning, resulting in early diagnosis and treatment ultimately decreasing transmission of TB, drug resistance, and potential death. Educators should be aware that TB patients' spirituality may be an important part of how they cope with having TB.

A larger scale study, conducted at multiple locations should be conducted to extend the findings of this small scale exploratory study. Further studies should be done to better determine what patient, healthcare provider and health care system factors might mediate relationships that may exist between lack of knowledge of TB, stigma and TB medication adherence. 


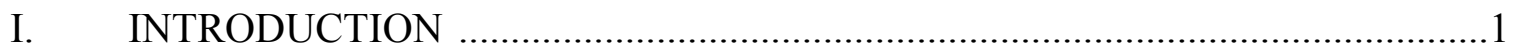

Background to the Problem ............................................................................

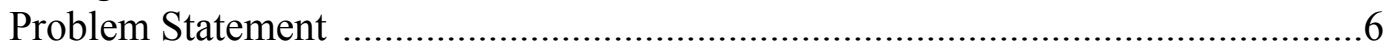

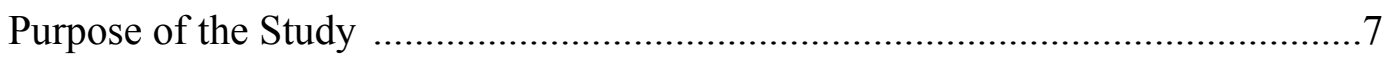

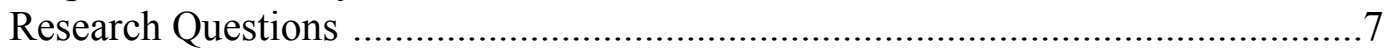

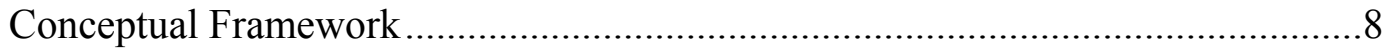

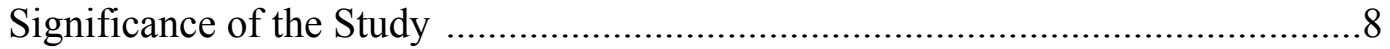

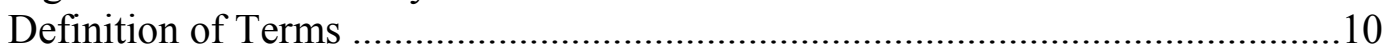

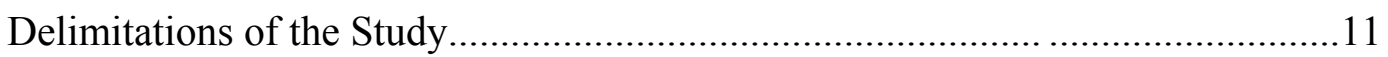

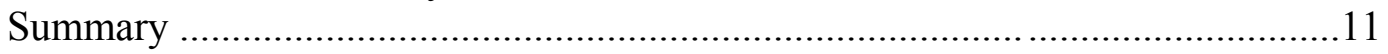

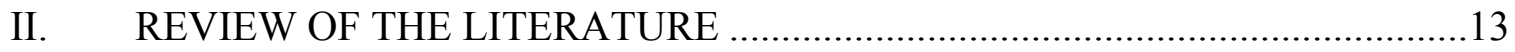

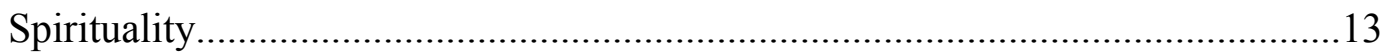

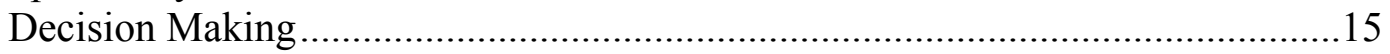

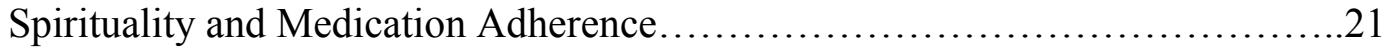

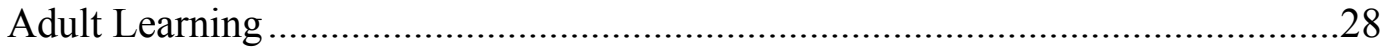

Patient and Staff Education.................................................................................

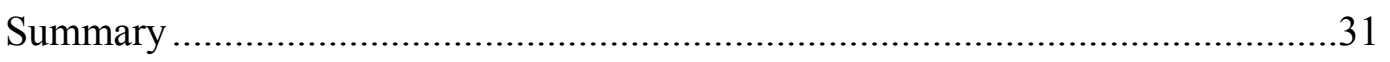

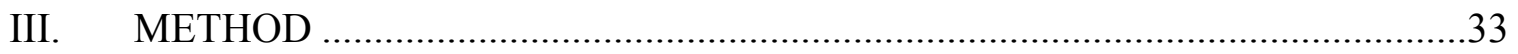

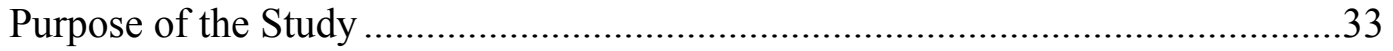

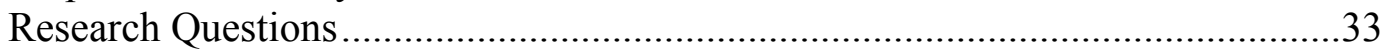

Mixed Method Research Design...........................................................................34

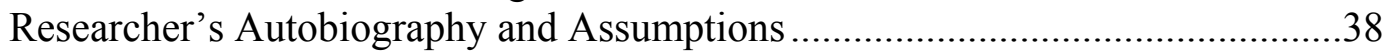

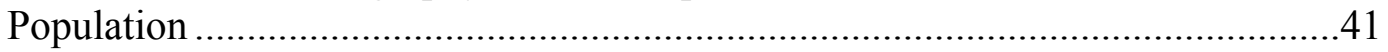

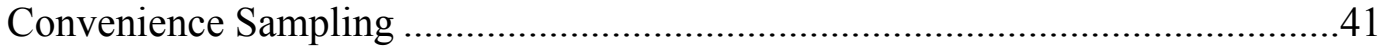

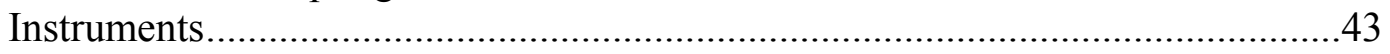

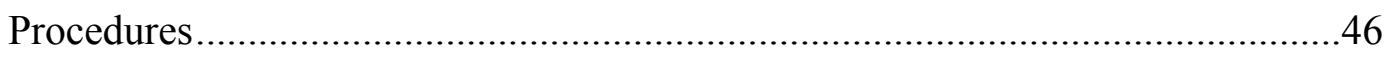

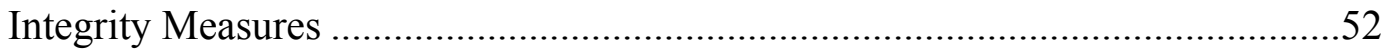

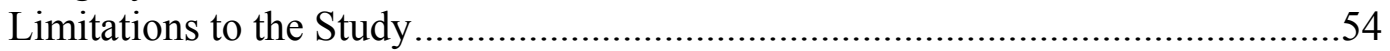

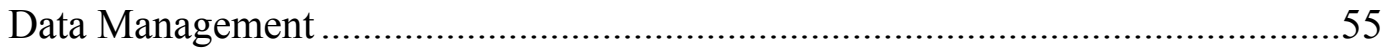

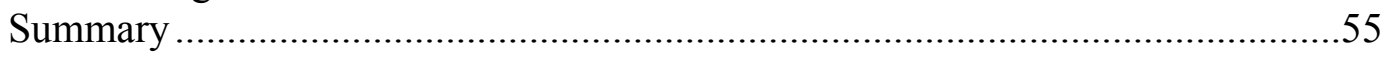

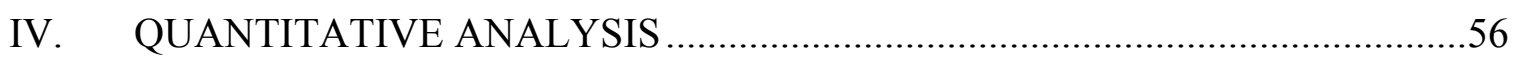

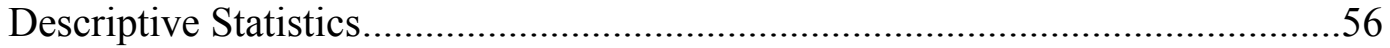

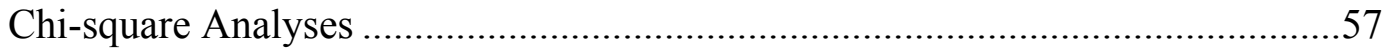

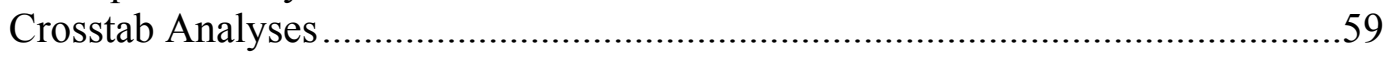

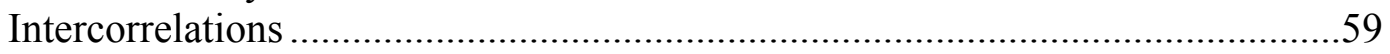

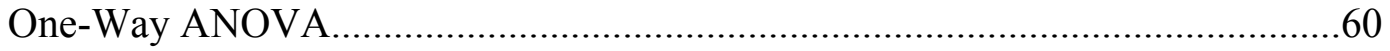

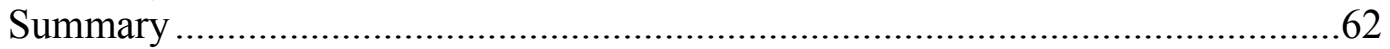




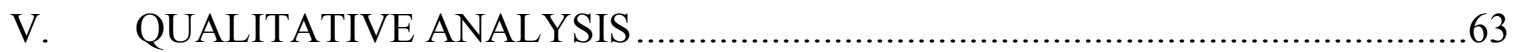

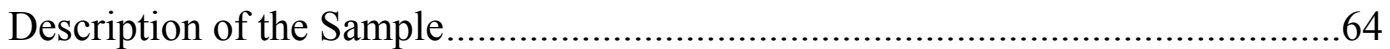

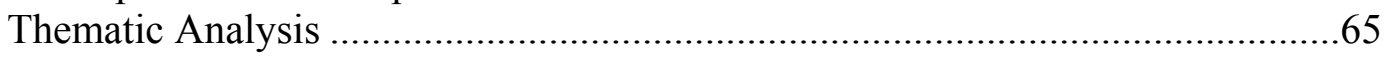

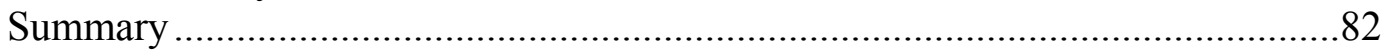

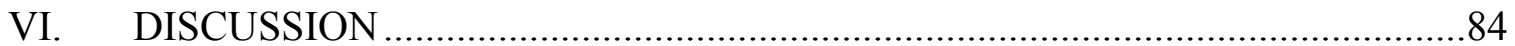

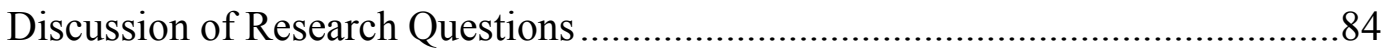

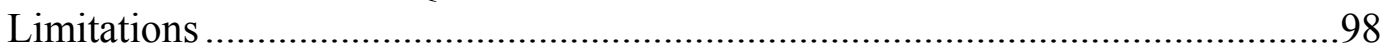

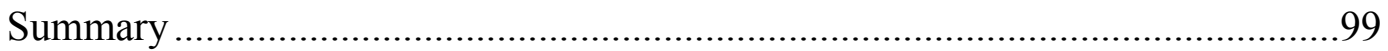

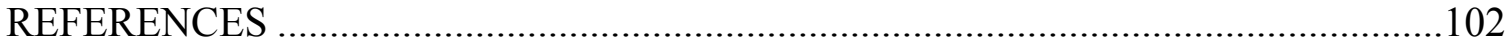

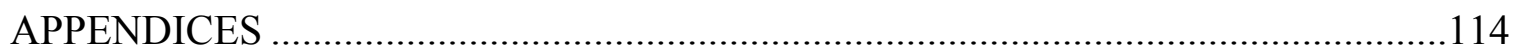

VITA 


\section{LISTS OF TABLES}

TABLES

PAGES

1. Summary of 2006 and 2007 TB Cases in Miami-Dade County........................

2. Chi-square Analyses of Study Variables....................................58

3. Crosstab Analyses of Sex, Language and Ethnicity..............................59

4. Crosstab Analyses of Marital Status, Language and Adherence......................59

5. Crosstab Analyses among Ethnicity, Language and Adherence....................60

6. Intercorrelations among Research Variables.................................61

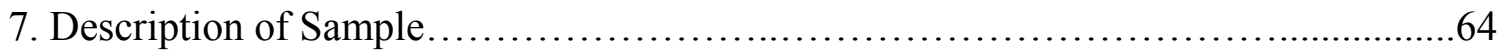

8. Description of Similarities and Differences across Groups........................82 


\section{CHAPTER I}

\section{INTRODUCTION}

This mixed method study explored the relationship between spirituality, knowledge, and tuberculosis (TB) medication adherence among African Americans and Haitians. This chapter presents the background to the problem, problem statement, purpose, research questions, conceptual framework, significance of the study, definition of terms, delimitations of the study, and a summary.

\section{Background to the Problem}

Tuberculosis (TB), an ancient, infectious airborne disease caused by Mycobacterium Tuberculosis, kills more people worldwide than any other infectious disease (Centers for Disease Control and Prevention [CDC], 2008; McDonald \& Reichman, 1998). Prior to the 1980s, the CDC predicted that TB would be eradicated. Unfortunately, since the late 1980s, TB has reemerged in the United States (Ginsberg, 1998). This is attributed to the increased prevalence of human immunodeficiency virus (HIV), the increased number of medically-underserved populations, as well as the increased number of foreign-born and older persons who were infected when they were young (CDC, 1998).

Worldwide, TB remains one of the leading causes of death from infectious diseases. An estimated 2 billion persons are infected with the bacteria causing TB. Annually, approximately 9 million persons become ill from TB; of these, 2 million die (CDC, 2008). In the U.S., an estimated 10 to 15 million persons are infected with the bacteria; but they do not necessarily become ill. Nationally, more than two-thirds of 
reported cases occurred in racial minority and ethnic groups such as African Americans and Haitians (CDC, 1992). This trend continued in 2007 (CDC, 2008).

Statistics based on race lump all persons of African descent together. These two groups of African descendents, African Americans and Haitians, have important spiritual beliefs and practices that are discussed in this study. African Americans are involuntary (nonimmigrant) minorities who have been enslaved and made to be a part of the U.S. society permanently against their will (Ogbu \& Simons, 1998). In this study, African Americans had to have family roots of more than three generations in the U.S. and when asked did not identify as having Caribbean ancestry. Haitians are voluntary (immigrant) minorities who willingly moved to the United States for better opportunities (education, jobs, and more political or religious freedom) than they had in their homeland (Ogbu \& Simons). In this study, Haitians had to be born in Haiti or have parents or grandparents born in Haiti. In national and state health statistics, Haitians are included in the Black race/ethnicity category. National statistical data categorizes TB cases into race/ethnicity groups of Black, White, Asian, Hispanic, American Indian/Alaska native, Native Hawaiian or Pacific Islander, multiple race, and unknown. The state of Florida categorizes TB cases into race/ethnicity groups of Black non-Hispanic, Hispanic (all races), White non-Hispanic, and other. In this study, the (Blacks) African Americans and Haitians were differentiated as separate race/ethnicity groups.

Because I was more familiar with the TB patient population and had access to TB patients in Miami, Florida, as compared Broward County, Florida, I focused this study in Miami. TB case data is presented at the national level, followed by the state of Florida, and concludes with Miami-Dade County. In 2007, 13, 293 new cases of TB were 
reported in the U.S., and of these cases, Blacks represented 3,454 (26\%; CDC, 2008). In the state of Florida in 2007, 980 TB cases were reported. Black non Hispanics, a group that includes Haitians, made up 378 (39\%) of Florida's 2007 TB cases. Haitians make up approximately 240,000 (4\%) of the population in Florida and account for $11 \%$ of the cases of TB (Coreil, 2001). In 2007, Miami-Dade County continued to rank first in TB morbidity in Florida. Miami-Dade County reported 182 new TB cases in 2007. During 2007, the number of TB cases in Miami-Dade for African Americans was 42 (24\%) and Haitians represented 37 cases (20\%; see Table 1; Miami-Dade County Department of Health, Tuberculosis Program, 2008).

Table 1

Summary of 2006 and 2007 TB Cases in Miami-Dade County

\begin{tabular}{ccccc} 
Year & Blacks & Whites & Hispanics & Haitians \\
\hline 2006 & 48 & 16 & 98 & 41 \\
2007 & 42 & 20 & 83 & 37 \\
\hline
\end{tabular}

Tuberculosis is a curable disease that requires long-term medication therapy. Failure to adhere to a treatment regimen creates potential for drug resistance, further spread of the disease, disability, and death. To break the cycle of TB infection and disease, appropriate diagnosis, treatment, and follow-up care by healthcare providers caring for all TB patients and in particular minority TB patients is necessary (CDC, 1998; Halverson, Mays, Miller, Kaluzny, \& Richards, 1997). However, in many cultures, the stigma of TB contributes to lengthy delays in seeking professional care and abandonment of treatment (Iiongo, 1994; Rubel \& Garro, 1992). 
Spirituality, as an element of culture expressed in the form of spiritual beliefs about health and disease treatment, may interfere with TB patients gaining knowledge of $\mathrm{TB}$, treatment, and medication adherence. Spirituality is defined as a relationship with a transcendent force that brings meaning and purpose to one's existence and affects the way in which one operates in the world (Armstrong, 1996; Mattis, 2005). Spirituality can also be defined as a way of finding meaning in life and a source for coping with life (Miller, 1995; Reed, 1992). Spirituality may be important in both the African American and Haitian cultures and may influence African Americans' and Haitians' healthcare decisions.

\section{African Americans}

In the African American community, $\mathrm{TB}$ is considered a dirty and mysterious disease that affects "bad" people; as a result, TB patients often experience stigmatization from family and friends (Jenkins, 1966; Kelly, 2000). Spirituality and religion have always been an important part of African American culture. In the African American culture, spirituality and spiritual beliefs provide comfort, support, and a coping mechanism. Family and extended family are important in the lives of African Americans. Families are the primary provider of information for transmission and expression of spirituality, religious practices, and values to younger generations. African Americans generally practice Christianity and the predominant church memberships are Baptist and Methodist (Pamphie, 2001; Pinkney, 2000; Trotter, 2001). African Americans' spirituality is important in medication adherence, understanding disease, coping, and making treatment decisions (Johnson, Elbert-Avila, \& Tulsky, 2005). 
Four major themes concerning religion/spirituality influence African Americans' decisions regarding health beliefs: (a) faith in divine healing with no human agency, (b) faith in divine healing through doctors, (c) faith in divine healing through health or religious behavior modifications, and (d) faith in acceptance of health outcomes (King, Burgess, Akinyela, Count-Spriggs, \& Parker, 2005). Most African Americans believe that God is responsible for physical and spiritual health and that doctors are God's instruments (Green, Lewis, Wang, Person, \& Rivers, 2004; Johnson et al., 2005; Lewis \& Green, 2000). Typically African Americans believe in a strong, intimate relationship with God and the ability to overcome health limitations through the power of prayer (King et al., 2005). Beliefs in God's control over their physical and spiritual health and the power of prayer may interfere with or encourage African American TB patients' medication adherence.

\section{Haitians}

In the Haitian community, TB is considered a shameful disease. Patients diagnosed with TB face social exclusion, rejection by family and friends, and placement in quarantine (Farmer, Robin, Ramilus, \& Kim, 1991; Heurtelou, 2001). Haitians believe that TB can be either naturally or supernaturally acquired and treated with biomedicine, natural medicine, and Voodoo (Miller, 2000). The majority of Haitians are Catholic, with a small number practicing other religions (Weidman, 1978; Chierici, 1991; Desrosiers \& Fleurose, 2002; Pamphile, 2001). Lower socioeconomic class Haitians are more likely to acknowledge the belief and practice of Voodoo, while upper socioeconomic class Haitians typically belong to the Catholic Church and deny practicing Voodoo (Chierici, 1991; Desrosiers \& Fleurose, 2002). 
Many Haitians believe in a spiritual world that is made up of both good and evil spirits (Weidman, 1978). Voodoo is a religion that originated in Africa and involves the worship of Gods of Voodoo, or Loas. Voodoo ceremonies traditionally conducted by a Voodoo priest known as a Houngan are devoted to the worship and invocation of the invisible world. Haitian beliefs about TB include a supernatural etiology in which TB is caused by sorcery (Coreil, 2001; Desrosiers \& Fleurose, 2002; Farmer, 2000). Haitians' belief in Voodoo gives patients a sense of optimism because Voodoo provides a sense of control over their destinies. These Voodoo-based spiritual beliefs have caused TB patients to abandon medical treatment (Farmer, 2000). If the patient abandons medical treatment and seeks alternative treatment, this creates a TB control challenge, such as further transmission of TB, drug resistance, and death.

\section{Problem Statement}

Adults requiring long-term medication and treatment can present a management challenge for healthcare providers. The healthcare literature explores cultural factors that affect health beliefs about TB. However, in the field of healthcare there is limited research on spirituality, aside from religion, and its relationship to health (Thoresen \& Harris, 2002). This study on African Americans' and Haitians' spirituality and its influence on patients' knowledge of TB and medication adherence may, provide up to date insights into TB patients' spirituality, knowledge of the disease symptoms and treatment, facilitate cultural training for healthcare professionals working with TB patients, and ultimately help to control the spread of TB in the community. 


\section{Purpose of the Study}

To shed light on spirituality and TB medication adherence, this mixed method study explored the relationship between spirituality, knowledge, and TB medication adherence among African Americans and Haitians. A correlational study was conducted using TB patients' medication adherence records and data from the Spiritual Perspective Scale to determine if a relationship exists between spirituality and TB medication adherence. In addition, insight from phenomenological interviews with a small subsample of TB patients was used to further examine the relationship between their spirituality and medication adherence. The phenomenon of concern was spirituality's relationship with knowledge and TB medication adherence among African Americans and Haitians.

\section{Research Questions}

The primary research question was: What is the relationship between spirituality, knowledge of TB, and TB medication adherence among African Americans and Haitians? The secondary questions were:

1. How do African Americans and Haitians perceive the role of spirituality in their lives after being diagnosed with TB?

2. How do African Americans and Haitians understand and learn about TB after diagnosis?

3. How do African Americans and Haitians understand the directions concerning TB medication adherence?

To examine the research question, the following research hypothesis was tested:

H1. There is no relationship between spirituality, knowledge, and TB medication adherence among African Americans and Haitians. 


\section{Conceptual Framework}

Although often used interchangeably, religion and spirituality have different meanings and may have different effects on the use of health services. Whether used together or separately, religion and spirituality provide a framework to make sense of the world and cope with life (Musgrave et al., 2002). Spirituality is a relationship with a transcendent force that brings meaning and purpose to one's existence and affects the way in which one functions in the world (Armstrong, 1996). Spirituality as a basic inherent quality in humans involves a belief in something greater than the self and a faith that positively affirms life (Musgrave et al., 2002). Spirituality can be individually focused and not associated with involvement in a supportive community (Koenig, George, Titus, \& Meador, 2003). Religions are differentiated by particular beliefs and practices, requirement of membership, and modes of social organization (Miller \& Thoresen, 2003). Religion and spirituality have been conceptualized to influence the development of each other. For example, religious practices encourage spiritual growth, and spiritual practices are often an important aspect of religious participation (Armstrong \& Crowther, 2002; Miller \& Thoresen, 2003). Religion and spirituality have been linked to health behaviors and health outcomes, such as decreased hospital length of stay and improved health (Hansel, Wu, Chang, \& Diette, 2004; Miller \& Thoresen, 2003; Westlake et al., 2002).

\section{Significance of the Study}

This study is significant for four reasons. First, TB is an infectious disease and nonadherence to medication can lead to new cases, multi-drug resistant $\mathrm{TB}$, or potential death. Additionally, the healthcare professionals' and the individuals' with knowledge of 
the disease and medication adherence are crucial for successful completion of medication therapy.

Second, patient education is one of the most important aspects of care provided in healthcare settings (CDC, 1994). Numerous barriers to patient education and treatment regimens exist in minority populations (CDC, 1994; Chackes \& Christ, 1996). Therefore, barriers to medication adherence in healthcare settings may be addressed and effective interventions can be developed to meet the patient's educational needs.

Third, TB tends to disproportionally affect minority and economically disadvantaged patient populations. Minority patients' culture and spirituality influence their perception of health (Korbin, Brinkley, Reebals, \& Singh, 1997; Moss, 1994). Exploring African American and Haitian patients' spirituality may enlighten healthcare providers to better understand health behavior influences of minority patients. Minority patients' spiritual health beliefs and practices need to be incorporated into health education materials to enhance factors associated with patient adherence to treatment regimen (CDC, 1994; Chackes \& Christ, 1996; Rubel \& Garro, 1992).

Fourth, healthcare providers can use this information to help adult learners understand the disease and make adjustments in their lives. Healthcare providers will have additional knowledge about African American and Haitian patients' spirituality and knowledge of TB as predictors of medication adherence. Findings from this study provide theoretical and empirical research extension to expand the cultural knowledge of healthcare educators and providers and allow them to discuss the effectiveness of understanding standard written or oral educational interventions provided to African Americans and Haitians diagnosed with TB. 


\section{Definition of Terms}

Adherence is the degree to which patients follow the prescribed interventions (CDC, 1994), for example, attending $80 \%$ of prescribed visits each month of treatment (Davidson, Schluger, Feldman, Valentine, Telzak, \& Laufer, 2000).

African Americans are involuntary (nonimmigrant) minorities of African descent who have been enslaved and made to be a part of the U.S. society permanently against their will (Ogbu \& Simons, 1998).

Directly Observed Therapy (DOT) consists of a county health department health service representative taking the TB medications to the patient and observing the patient ingesting the medication (CDC, 2000).

Haitians are voluntary (immigrant) minorities who willingly moved to the United States from Haiti because they expect better opportunities (education, jobs, more political or religious freedom) than they had in their homeland (Ogbu \& Simons, 1998).

Knowledge is defined as human beings' understanding about reality through mental correspondence, personal experience, and emotional affection with outside objects and situations. Knowledge may be explicit, implicit, and emancipatory (Yang, 2003). In this study all three knowledge facets are relevant.

Phenomenology is a qualitative research method used to explore how human beings make sense of experience and transform experience into consciousness, both individually and as shared meaning (Patton, 2002),

Phenomenon can be an emotion, relationship, such as spirituality, marriage, job, program, organization, or a culture (Patton, 2002). Spirituality will be the focus of the study. 
Religion is a group activity that involves specific behavioral, social, doctrinal, and denominational characteristics (Armstrong \& Crowther, 2002).

Spirituality is a relationship with a transcendent force that brings meaning and purpose to one's existence and affects the way in which one operates in the world (Armstrong, 1996; Mattis, 2005).

$T B$ disease is diagnosed when as a person becomes symptomatic, has a positive culture for TB, and may be infectious (CDC, 2004).

$T B$ infection occurs when a person has been exposed to the TB germ but is not symptomatic or infectious (CDC, 2004).

\section{Delimitations of the Study}

Because I was more familiar with the TB patient population and had access to TB patients in Miami, FL, I focused this study in that area. Also, I only looked at African American and Haitians individuals who reside in Miami and are under treatment for TB. Measuring medication adherence in the healthcare field has not been clearly defined. The lack of a valid method for measuring non-adherence is a major barrier in adherence research (Sackett, 1976; Vermeire, Hearnshaw, \& Van Royen, 2001).

\section{Summary}

This chapter introduced the study including the background to the problem, problem statement, purpose of the study, research questions, and conceptual framework. The significance of the study, definition of terms, and delimitations of the study were also discussed. Chapter 2 consists of a literature review to support the study. Chapter 3 discusses the methodology that consisted of correlational research design and 
phenomenological research design, data collection procedures, and data analysis. Chapter 4 presents the findings from the quantitative data analysis on the relationship between spirituality and TB medication adherence among African Americans and Haitians. Chapter 5 presents the findings from the qualitative data analysis. Then Chapter 6 consists of conclusions from the research with recommendations for future research and intervention in the adult education field. 


\section{CHAPTER II}

\section{REVIEW OF THE LITERATURE}

A review of the literature was conducted to explore the relationship between spirituality and TB medication adherence among African Americans and Haitians. This chapter is organized by the following headings: spirituality, decision making, spiritual beliefs and medication adherence, adult learning, patient and staff education, and a summary.

\section{Spirituality}

The relationship between spirituality and a transcendent force brings meaning and purpose to individuals' lives, affects the way in which individuals' operate in the world, and serves as a source for coping with life (Armstrong, 1996; Mattis, 2005; Miller, 1995; Reed, 1992). Spirituality involves faith or willingness to believe, a search for purpose in life, a sense of connection with others, or a transcendence of the self, resulting in a sense of inner peace and well-being (Delgado, 2005; Mattis, 2005). The concept of spirituality serves as a way for people to interpret their lives and experiences (Johnson et al., 2005). Spirituality may be further conceptualized as the beliefs, behaviors, characteristics, and ultimately experiences that are grounded in and further developed by the individual's relationship with God or a transcendent force (Mattis, 2005) and is a constantly present resource in an individual's health (Reed, 1991). This spirituality resource guides individuals in making sense of and coping with health concerns. Thus, spirituality helps individuals find meaning in illness, suffering, and death (Dyson, Cobb, \& Forman, 1997). This spirituality resource may have a positive or negative influence on an individual's decision making about adult learning and medication adherence. Some aspects of 
spirituality are observable and displayed in spiritual practices including praying, religious reading, and attending religious services (Armstrong \& Crowther, 2002; Miller, 1995; Miller \& Thoresen, 2003; Musgrave, Allen, \& Allen, 2002).

Although spirituality and religion are connected, they are uniquely different. Spirituality is usually understood as an individual phenomenon (Thoresen \& Harris, 2002). Ultimately, spirituality is part of an individual's lifelong search for meaning or a search to make sense of everyday routines (English, Fenwick, \& Parson, 2003; Miller, 1995; Reed, 1992). Religion is seen as fundamentally a social phenomenon. Religion is generally a group activity with specific behavioral, social, doctrinal, and denominational characteristics (Armstrong \& Crowther, 2002). Religion involves participation in a formal church, organization, or religious affiliation with a particular faith or denomination that includes commitment to attend worship services and belief in traditional creeds or orthodoxy (Armstrong, 1996). Despite the differences, both terms provide a framework for making sense of the world and coping with life (Mattis, 2005; Miller, 1995). Although both religion and spirituality are deeply intertwined, for the purpose of this research, I focused on spirituality, an individually focused phenomenon. The next section discusses empirical research conducted on the influences of spirituality in the lives of TB patients.

Hansel, Wu, Chang, and Diette (2004) conducted four focus groups and individual interviews with 10 TB patients, 9 nurses, and 4 physicians who treated TB patients to describe TB impact on patients' quality of life. In that study, quality of life was defined as the person's perceptions of his or her physical and mental health, which covers broad domains such as physical, psychological, economic, spiritual, and social 
well-being. The participants were mainly African American. Patients reported that having TB increased their spirituality and improved their life perspectives. Patients reported that being diagnosed with TB enhanced their spirituality through increased faith in God, which provided considerable support during their illness. Patients experienced an increased sense of spirituality through reading, reflecting, and praying. During the time patients were in isolation, patients reported that they had the opportunity to think and learn to listen to God. According to the patients, through their increased spirituality, they became wiser, more cautious, and more concerned about their health. Interestingly, interviews revealed that healthcare providers did not talk about the role of spirituality in their patients' lives. This suggests that there was a missed educational opportunity for the healthcare providers to recognize the role of spirituality in their patients' lives and then incorporate spirituality into their educational interventions.

\section{Decision Making}

TB patients' knowledge and beliefs about TB influence their decisions to adhere to medical treatment (San Sebastian \& Bothamley, 2000). This section presents the theory of reasoned action (Ajzen \& Fishbein, 1980), self-regulative system theory (Leventhal \& Cameron, 1987), and the Health Belief Model (Rosenstock, 1966). These two theories and a model may help healthcare providers understand patients' decision making about TB medication adherence. This discussion about theories that influence decision making is followed by a discussion about factors that affect medication adherence among patients in general and among Africans, African Americans, and Haitians, in particular. 


\section{Theory of Reasoned Action}

The theory of reasoned action (Ajzen \& Fishbein, 1980) explains the relationship between beliefs, intentions, and actions. Individuals choose to take or not to take actions based on beliefs and values learned in life situations or from significant others. The individuals' spiritual beliefs may interfere with the healthcare provider prescribed treatment plan. According to this theory, patients who leave the healthcare provider's office with the intention to take their prescribed medication have a greater likelihood of complying with their TB medication regimen. Based on this theory, healthcare providers should ask the TB patient if he/she believes that TB medications will have an effect, either positive or negative, and if the patient intends to follow the treatment regimen.

\section{Self-Regulative System Theory}

Self-regulative system theory (Leventhal \& Cameron, 1987) views the patient as an active problem solver. Patients have the ability and are willing to self-regulate their behavior to obtain or maintain a healthy state. Compliance (adherence) behavior depends on the patient's cognitive understanding of current health status, the goal state, plans for changing the current state, and techniques or rules for appraising processes. Compliance also depends on the clarity of communication between the patient and the healthcare provider during the health education process. The patients' spirituality may enhance or interfere with the communication between the patient and healthcare provider. For example, the patient's intention to change health behaviors or listen to the healthcare provider may be based on the patient's perception of the results of their spiritual practices. 


\section{Health Belief Model}

The Health Belief Model (HBM), developed by Rosenstock (1966) and later modified by Becker (1974), explains health behaviors. The HBM has helped researchers understand adherence with treatment and medication. This model includes specific health beliefs about susceptibility, severity of illness or condition, and diagnosis. Health decisions are affected by health beliefs, experience, knowledge, social interaction, and sociodemographic factors. When given the appropriate information, patients are capable of assessing susceptibility and efficacy and making a decision to select and comply with specific recommendations or treatment plans based on their own risk/benefit analysis. Inappropriate or insufficient knowledge regarding potential hazards of not engaging in a particular health behavior or regarding the benefits from engaging in health behaviors results in the patient's nonadherence to the treatment regimen (Leventhal \& Cameron, 1987).

Worldwide TB medication adherence rate is estimated to be $50 \%$, which can cause medical and psychosocial complications, reduce patients' quality of life and waste health care resources for patients who do not comply (WHO, 2003). A metaethnographic study conducted over a 10-year period on patients' views of medication adherence revealed the following findings: (a) evaluation of medication and difficulties, (b) medicines and identity, and (c) and the way patients take their medicines (Pound et al., 2005).

\section{Evaluation of Medication and Difficulties}

The most common means of evaluation was based on benefits versus cost of taking medications. Patients took their medication to obtain relief from symptoms, avoid 
hospitalization, prevent disease progression, and be normal. Acceptability based on the medication regimen fitting in the daily lifestyle was another factor that patients evaluated to determine if they would take their medication. Lack of knowledge and communication between physician and patients caused patients to have difficulty in evaluating their medication (Pound et al., 2005).

\section{Medicines and Identity}

Acceptance is a major factor in medication adherence. For example, patients with asthma tend to downplay the significance of their illness (Adams, Pill, \& Jones, 1997). This may be same with TB patients, who present to their healthcare provider with a complaint of a cold or pneumonia. Acceptance or denial of their illness may influence patients' decisions to seek medical care and take medications. Patients may want to feel normalcy in their lives. Patients may equate taking medications with having an illness. Patients in denial tend to feel the medicines do not work and avoid taking the medicines and keeping clinic appointments. Medication such as psychiatric medication caused patients to be labeled as different; therefore, patients who feared disclosing their health status and being stigmatized delayed or did not take their medication.

$\mathrm{TB}$, as well as conditions such as asthma, can be a life threatening condition in which medication is the major form of treatment. In a phenomenological qualitative study on asthma in which medication is also the major form of treatment, Walsh, Hagan, and Gamsu (2000) identified three types of behaviors of patients who did not take their asthma medication as prescribed: (a) denial, (b) avoidance, and (c) depression. Patients in denial did not want to see themselves as having an illness or to manage the illness and did not take prescribed medications. Patients in avoidance felt the medications did not help, 
so they were nonadherent with their medications and clinic appointments. Depression may have resulted from a sense of sadness in relations to a change in life and restrictions placed on them.

Patients with TB are fearful of disclosing their illness to others due to the stigma that is attached to the disease (Coreil, 2001; Handel, Chang, \& Diette, 2004; Heurtelou, 2001; Ilongo, 1994; Kelly, 1999). To avoid disclosing their illness to others, including family members, patients may not take their medications in public or at home. Patients feel that certain medications identify them as having conditions such as schizophrenia and HIV and make them feel stigmatized (Laws, Wilson, Bowser, \& Kerr, 2000; Usher, 2001). TB patients may be labeled by the medication they are taking.

\section{The Way Patients Take Their Medicines}

Patients take medication through imposed compliance or voluntary. Imposed compliance is the result of pressure to take medication exerted by relatives or healthcare professionals. This imposed compliance in TB control is done through TB control laws and directly observed therapy that is provided by a healthcare worker. Either way, patients want to minimize the amount of medications prescribed. To minimize the amount of medications prescribed, patients replace or supplement medication with nonpharmacological treatments such as herbs, home remedies, and root doctors (Pound et al., 2005). Patients resist taking medications because of concerns about the medicines.

Healthcare providers must recognize patients' concerns and develop safe medicines and identify alternative treatments patients prefer to use instead of medicines.

Cultural health beliefs about health and treatment of a disease may interfere with TB treatment and contribute to nonadherence. In many cultures, the social stigma of TB 
and discrimination by healthcare professionals contribute to abandonment of treatment and lengthy delays in seeking professional care (Ilongo, 1994; Rubel \& Garro, 1992). Foreign-born immigrants and refugees diagnosed with $\mathrm{TB}$, fearing that the disease may result in legal actions such as deportation when picking up medication refills, present a medication adherence challenge for healthcare providers (CDC, 1994). In addition, immigrants frequently move out of their current place of residence or return to their countries of origin during treatment (Cummings, Mohle-Boetani, Royce, \& Chin, 1998; McCleod, 1998). Unfortunately, these patients view adherence to TB treatment as a low priority compared to the demands of day-to-day existence (Kitazawa, 1995). Due to the amount of time off from work needed for follow-up clinic visits, patients fear the possible loss of employment or income.

To identify patients' characteristics associated with TB medication adherence, McDonnell, Turner, and Weaver (2001) conducted a correlational study of 62 adults. The majority were African Americans, who were diagnosed with active TB and placed on a treatment regimen. Patients completed a sociodemographic form and self-reported questionnaire, which measured patients' general tendency to adhere to the TB medication regimen. Patients were more likely to adhere to the TB medication regimen based on them having the following: (a) satisfaction with interpersonal care, (b) good perception of the effectiveness of the medication, and (c) support from family and friends.

Sociodemographic factors such as alcohol use, educational level, and income level influenced patients' intention or practice to take TB medication. Those patients who used alcohol had lower intentions to adhere to their TB medication regimens. The patients with higher education and income had higher intentions to adhere to their TB 
medication regimen. The perceived presence of supports and the absence of barriers to adherence strengthened the patients' intentions to adhere to their medication regimens. An individual's intention to adhere to a medication regimen is directly influenced by the belief in the medication benefits and usefulness. Adherence was found not to be related to duration of time taking anti-TB medications. However, McDonnell et al. (2001) found social problems such as homelessness and drug abuse to be associated with nonadherence to TB medication regimens.

\section{Spirituality and Medication Adherence}

People from African American and Haitian cultures have roots in African culture that influences many of their attitudes, health and spiritual beliefs, and medication adherence. African Americans and Haitians have similar attitudes and health and spiritual beliefs that are discussed in the following section. African, African American, and Haitian spirituality and medication adherence are discussed in this section.

\section{African Spirituality and Medication Adherence}

To describe the community's beliefs and experiences about TB patients and how these beliefs and experiences affect medication adherence, Edginton, Sekatane, and Goldstein (2002) used structured interviews with 303 African TB patients and qualitative focus group interviews with 14 different groups consisting of TB patients, community members, local traditional healers, community leaders, student nurses, and road workers. People in the community believed that TB was the result of breaking cultural rules and consequently TB could only be treated by traditional healers. Interview results revealed that $63 \%$ believed TB was due to disobeying traditional rules, and $71 \%$ said that $\mathrm{TB}$ was sexually transmitted. For example, patients attributed TB to eating food prepared by a 
woman who had broken traditional rules. Contributing factors influencing TB medication adherence included the stigma of TB, instructions to abstain from sex while on treatment, difficulties accessing health services, long waiting periods at the clinics, and disrespectful local health workers (Edginton et al., 2002). In the African culture, stigma attached to the disease is a common problem. TB is known as a disease of "bad" people. Because of the association with drinking and poverty, individuals with TB are perceived as having infringed upon cultural norms. The help to reduce the stigma and miseducation that occurs with TB patients, health educators and healthcare providers should learn about their patients' beliefs. The findings from that study support the need for educational interventions to encourage medication adherence and control the transmission of TB.

To identify beliefs and attitudes regarding health seeking behaviors among TB patients, Steen and Mazonde (1999), using focus groups, interviewed 212 TB patients in Botswana, South Africa. Interviews and medical record reviews revealed 50\% reported lack of knowledge of what caused TB. The majority of patients (90\%) believed that medical doctors can cure TB. However, before TB medication was started by medical doctors, over half the patients (52\%) had tried alternative treatments, such as traditional healers, faith healers, and home remedies. Also, after TB medication was started, $47 \%$ of patients continued to visit a traditional healer or faith healer. The patients revealed the reason for seeing a traditional healer or faith healer was for cleansing and to identify the cause of the disease. Additionally, it was a cultural tradition. Based on individual interviews and focus groups interviews, an association was found between beliefs in breaking traditional rules and visits to traditional healers as the cause for TB. The lack of knowledge about the cause of TB is evident by the practice of spiritual beliefs and ritual 
before seeking medical care. This lack of knowledge among TB patients is an opportunity for improved health education and collaboration between healthcare providers, spiritual leaders, and TB patients. Healthcare providers' educational interventions should be directed at the spiritual leaders in the community as well as the TB patients.

In two Western Cape, South African, suburbs with high incidence of TB, Ellis, Beyers, Bester, Gis, and Donald (1997) interviewed 23 patients and their household members to determine patients' belief systems, household and community practices, knowledge about TB and adherence to TB medication, and service utilization. Severely deprived patients reported limited perceptions of what constitutes personal and public health and how to remain healthy. For example, participants reported causes of TB include cold weather, smoking, malnutrition, or poor eating habits. These reported beliefs clearly show the participants' lack of knowledge about TB. Some participants had no insight into TB or the role of overcrowded households in TB transmission. Participants rarely visited a doctor; they would first use some home remedy or over the counter medication. Elderly residents reported using traditional herbal remedies and religion as a back-up to medical care. Most of the participants had limited understanding of the need to complete $\mathrm{TB}$ treatment and expressed a wish to be cured of TB as motivation for treatment adherence. Some participants reported intentional nonadherence to remain sick and, thus, qualify for state social security support. The decision to be nonadherent to TB medication points out the adjustments the residents in the South African town made to obtain financial assistance in a community with high unemployment and poverty. Study participants expressed a basic belief and trust in the professional medical care system and the biomedical model of care. 
Metcalf, Bradshaw, and Stindt's (1990) study with 24 unemployed women in the Cape Town vicinity found that the women had knowledge about TB symptoms and treatment, and they understood the need for TB medication adherence, insisting that they wanted to be cured from TB. However, their health behaviors contradicted this. For example, $32 \%$ of TB patients took less than $75 \%$ of their TB medication, despite the availability of options for supervision of therapy. Knowledge about TB symptoms did not ensure appropriate health behaviors when people were developing symptoms of and were being diagnosed with TB. Women failed to keep clinic appointments for evaluation. Also, $49 \%$ percent of TB cases were in children under age 16 , which suggested that some adults in the community had untreated TB. In this South African community, as in other African communities, stigma attached to TB and fears of social isolation were major concerns for the women. Unfortunately, people living in destitute conditions may not perceive adherence to TB medication and treatment as their highest priorities. This demonstrates the need for community education to dispel misconceptions about TB transmission, eliminate undue fear, and lessen the social stigma attached to TB. African Americans' and Haitians' spiritual beliefs and practices have some similarity to those of Africans. A discussion about how Africans' spirituality and medication adherence are similar to African American and Haitian spirituality and medication adherence follows.

\section{African American Spirituality and Medication Adherence}

In the African American culture, spirituality is important in understanding and coping with illness and may influence treatment preferences throughout treatment. African Americans' affirmation of God's sovereignty over their health is evident by spiritualizing rather than medicalizing their health beliefs (King et al., 2005). African 
Americans use meditation, prayer, and a belief or faith in a higher power such as God's will for them. African American families transmit faith-based health beliefs and model health behaviors across generations.

Johnson and colleagues (2005) found 27 studies that addressed spiritual beliefs and their influence on medical treatment choices of African Americans when they encounter illness. The authors found three themes: (a) the importance of spiritual beliefs and practices, such as prayer, in coping with illness; (b) the power of spiritual beliefs and practices to promote healing; and (c) the belief that God is responsible for physical and spiritual health. Spiritual beliefs were the most important factor in healing, along with the belief in miracles and faith healing. Interestingly, the participants reported a belief in the physician as God's instrument to promote healing.

African American TB patients from Chicago reported experiencing stigmatization and isolation from family and friends (Kelly, 2000). This stigmatization is similar to the stigma experienced by Africans in the previous section. After they were diagnosed with $\mathrm{TB}$, most patients felt that family and friends avoided or shunned them. In turn, they isolated themselves from family and the community and chose not to disclose their illness. These factors, such as stigmatization and isolation, may contribute to further transmission of TB and nonadherence to TB medication and treatment.

A survey examining TB knowledge among 32 African American female drug users with a history of active TB found that $75 \%$ displayed a lack of knowledge about TB (Wolfe et al., 1995). Forty percent of the sample had low medication adherence scores. Given the high level of perceived stigma of TB and misinformation regarding its transmission among drug users, drug use would likely interfere with TB medication 
adherence. The association between lack of knowledge and medication adherence is evident in this study and requires educational intervention to prevent further transmission of TB.

A seminal study about community beliefs and feelings about TB revealed differences in perceptions of TB to be associated with educational level, social class, and ethnic-group membership (i.e., African Americans, Latinos, and Anglos; Jenkins, 1966). Four hundred thirty-six adults were selected and given a questionnaire to complete. African Americans believed TB to be found in their community more commonly than did Anglos or Latinos. African Americans perceived TB to be a mysterious, fast-moving, powerful disease, as well as embarrassing and dirty. Anglos and Latinos viewed TB as less of a threat. Anglos and Latinos shared a belief that TB was a slow-moving, weak disease that was well understood by science. African Americans share a similar belief as Africans that TB is considered a dirty disease with attached social stigma, which attacked bad people. Anglos and Latinos had a neutral moral evaluation of people with TB.

\section{Haitian Spirituality and Medication Adherence}

In rural Haiti, poverty was found to be a primary factor in nonadherence resulting in TB treatment failure. To capture the experience of TB in Haiti, Farmer, Simon, Ramilus, and Kim (1991) conducted open-ended interviews with two groups of $30 \mathrm{~TB}$ patients in each group to gather the patients' understanding of TB. The interviews revealed that cultural factors, similar to those of the African and African American population, such as the universal stigma attached to TB, contributed to nonadherence of treatment. In addition, spirituality was shown to influence patients' knowledge about TB 
and medication adherence. Interestingly, $85 \%$ of patients believed sorcery to be the cause of TB, which also led to nonadherence to medical treatment (Farmer et al., 1991).

A cultural feasibility study on Haitians living in South Florida was conducted to identify factors that might influence utilization of screening and treatment services for latent TB infection (Coreil, Lauzardo, \& Heurtelou, 2004). Data from five focus groups revealed beliefs that TB is caused by rapid chilling of the body, physical or psychological stress, and sorcery. Cough was reported as the main symptom of TB along with the body going from very hot to cold. Many participants considered TB to be a less serious illness in the United States than in Haiti. In the Haitian community, people with TB often encountered negative family reactions to their diagnosis. Unfortunately, due to negative publicity in the media that Haitians were carriers of Acquired Immune Deficiency Syndrome (AIDS), Haitians felt that they were being singled out for testing and were treated in humiliating ways by the healthcare community, leading to anger and resentment (Coreil et al., 2004).

During the screening process, if a person had TB symptoms and tests were negative, the person believed in a supernatural cause and sought a houngan (spiritual healer; Coreil et al., 2004). If the cause was believed to be supernatural, people were less discriminated against, received more sympathy, and were viewed by other Haitians with less suspicion as the targets of an evil act. The participants did not distinguish between infection and disease, understanding that TB infection was the "little brother of TB disease" (Coreil et al., 2004, p. 5). Long duration of treatment (6-24 months), cultural factors, misunderstanding of the effects of the preventive vaccine Bacille CalmetteGuerin (BCG), mistrust of the doctor, and fear of having TB-influenced TB medication 
adherence (Coreil et al, 2004). Haitians' interpersonal relationships and their recognition of emotional/spiritual issues have been shown to influence TB medication adherence.

\section{Adult Learning}

To explore how adults learn, Yang's (2003) Holistic Theory of Knowledge and Learning is used to examine the interaction of spirituality with learning. In this section, I discuss the adult learning process and the relationships among learning, meaning making, and spirituality. First, The Holistic Theory of Knowledge and Learning is presented, followed by the relationship between learning and spirituality.

\section{Holistic Theory of Knowledge and Learning}

Yang's (2003) Holistic Theory of Knowledge and Learning was developed to help adult educators better understand the learning process of adult learners. Holistic learning theory includes three knowledge facets: explicit, implicit, and emancipatory. The learning process consists of three independent activities: (a) knowledge creation, (b) knowledge acquisition, and (c) knowledge transformation. "Knowledge is defined as human beings' understanding about reality through mental correspondence, personal experiences, and emotional affection with outside objects and situations" (p. 108). Learning is a change in an individual's behavior, cognition, or affect that occurs as a result of interaction with the environment (Yang, 2003). Learning is also a process whereby knowledge is created, acquired, transformed, converted, or utilized in a different form from its original form (Yang, 2003). Holistic Theory of Knowledge provides a framework to understand the interactions among different types of knowledge. Knowledge facets are various aspects of the way in which we know and understand the 
physical, social, emotional, and spiritual world (Yang, 2003). The three knowledge facets (i.e., explicit, implicit and emancipatory) and learning processes are defined below.

Explicit knowledge is the cognitive component of knowledge that represents an individual's understanding of reality. Examples of explicit knowledge are theories, models, and formulas used by individuals in the learning process. Explicit knowledge represents the participants' understanding of TB symptoms and treatment plan in this study. Implicit knowledge refers to knowledge underlying behaviors, actions, experiences, and practices and tacit understandings of the individual. In this study, implicit knowledge was measured by medication adherence, the health behavioral component of this study.

Emancipatory knowledge represents emotions, values, feelings, affect, spirituality, personal aspirations, and visions for the individual who seeks freedom. In this study, emancipatory knowledge was shown by response on the Spiritual Perspective Scale (see Appendix A). Interviews with African American and Haitian TB patients revealed how they understand and learn about TB. Emancipatory knowledge determines the individual's motivation for learning. The three knowledge facets are complementary, indivisible, and necessary components of the holistic theory of knowledge (Yang, 2003). Each knowledge facet provides support for the existence of the other facets.

Knowledge creation is a process of learning where new understanding is formed, leading to a new format of explicit knowledge. Knowledge acquisition is the process of gaining knowledge from an external source, such as a lecture or reading a new book. Knowledge transformation refers to a process where learning is reformatted from one knowledge facet into another (Yang, 2003). For example, a change in spiritual belief 
(emancipatory) and knowledge (explicit) becomes a new habit such as medication adherence (implicit).

\section{The Relationship between Learning and Spirituality}

Adult education has had strong religious and spiritual foundations, and many movements in adult education began in religious or spiritual organizations (Tisdell, 2003). The New York Chautauqua movement was founded to improve teaching in Sunday schools and today continues to feature spiritual study as part of its educational programs (English, Fenwick, \& Parsons, 2003). Spirituality is viewed as a means by which adult learners acquire knowledge and create meaning (Dirkx, 1997, 2001). Spirituality has been shown to inform, guide, and change cognitions, behaviors, and emotions in adult learners (Gallagher, 2006). Education, in turn, gives meaning to life and assists in the growth of the human spirit (English et al., 2003).

Spirituality is usually present in the learning environment because adult learners usually bring it up or refer to spiritual issues or rituals to help them make some change in their environments (Tisdell, 2001). Also, Dirkx (1997) suggested that in learning, one should recognize spiritual issues are already present in our relationships and experiences in order to recognize spirituality in the teaching and learning environment. Emotions related to spirituality play an important role in adult learning because emotions can either interfere with or encourage learning. "Personally significant and meaningful learning is grounded in and is derived from the adult's emotional, imaginative connection with the self and with the broader social world" (Dirkx, 2001, p. 64). Therefore, the process of meaning making in adult learning is essentially imaginative and extra-rational. 
Emotions play an important role in how adults interpret and make sense of the events in their lives. Spirituality has been viewed as a means of finding meaning in life and providing a source for coping with life (Miller, 1995; Reed, 1992). Spirituality works together with the emotional, cognitive, unconscious, and symbolic domains to help adults construct meaning (Tisdell, 2001). This quest to find meaning is a motivating force behind intellect and emotions that can interfere with or enhance learning.

\section{Patient and Staff Education}

The purpose of patient education is to increase the competence and confidence of patients for self-management (Bastable, 2003). The purpose of staff education is to increase the competence and confidence of health care staff to provide quality care to patients. Patient education has the potential to promote adherence to TB treatment plans. Greater knowledge of disease and its treatment is often associated with improved adherence (Oser, 2006). Because spirituality is usually present in the learning environment, the provision of accurate and relevant information is important in helping both patients and healthcare staff explore personal beliefs, values, behaviors, and attitudes. The teaching incorporates the behavioral, cognitive, and affective domains of knowledge and promotes critical thinking in the patients (Hakala, 2007). The provision of accurate knowledge to patients and staff helps to change their inaccurate beliefs about TB and medication adherence and allow new knowledge to be learned.

\section{Summary}

The literature review examined the role of spirituality in the African American and Haitian cultures, decision making, the relationship between spirituality and TB medication adherence, adult learning, and patient and staff education. Studies have 
revealed factors such as stigma, poverty, cultural beliefs, spiritual beliefs, and lack of knowledge have a role in TB medication adherence among African Americans and Haitians. Healthcare providers must find new and innovative means to educate African American and Haitian TB patients to follow the recommended treatment regimen until they are cured. Health education provided in a culturally sensitive manner may empower African American and Haitian TB patients with information, thus influencing their beliefs and decision making skills about TB and TB medication adherence. In chapter 3, the research method (research design, setting, sampling, data collection, and analysis procedures), including the use of correlation statistics and phenomenology, is discussed. 


\section{CHAPTER III}

\section{METHOD}

This chapter begins with the purpose of the study, research questions, and hypotheses repeated from chapter 1 . Next, the mixed method research design is discussed, followed by my autobiography, assumptions, and journal related to the study. Then, a description of the population, convenience sampling, instruments, procedures for data collection and analysis, limitations, and data management are provided.

Purpose of the Study

To shed light on spirituality and TB medication adherence, this mixed method study explored the relationship between spirituality, knowledge, and TB medication adherence among African Americans and Haitians. A correlational study was conducted using TB patients' medication adherence records and data from the Spiritual Perspective Scale to determine if a relationship exists between spirituality and TB medication adherence. In addition, insight gained from phenomenological interviews with a small subsample of TB patients was used to further examine the relationship between their spirituality and medication adherence. The phenomenon of concern was spirituality's relationship with knowledge and TB medication adherence among African Americans and Haitians.

\section{Research Questions}

The primary research question was: What is the relationship between spirituality, knowledge of TB, and TB medication adherence among African Americans and Haitians? The secondary questions were: 
1. How do African Americans and Haitians perceive the role of spirituality in their lives after being diagnosed with TB?

2. How do African Americans and Haitians understand and learn about TB after diagnosis?

3. How do African Americans and Haitians understand the directions concerning TB medication adherence?

To examine the research question, the following null hypothesis was tested:

H1. There is no relationship between spirituality, knowledge, and TB medication adherence among African Americans and Haitians.

\section{Mixed Method Research Design}

Although positive associations between spirituality and health outcomes have been established, the underlying mechanisms are not well understood (Thoresen \& Harris, 2002). To obtain a richer and more accurate understanding of the issues around spirituality and health outcomes, a mixed method research design was used (Creswell, 2003). A mixed method research design combines the characteristics of quantitative and qualitative traditions, resulting in inferences grounded in participants' lives (Greene \& Caracelli, \& Graham, 1989). To provide better opportunities to answer the research questions, a mixed method research design has the following three advantages: (a) can answer questions that the other research methods cannot, (b) provides better inferences, and (c) allows for presenting a greater diversity of divergent views (Tashakkori \& Teddlie, 2003). 
First, mixed method research allowed me to verify a conceptual framework in this study which could not be addressed by one method. This method allowed me to test my quantitative hypothesis and explore in greater detail the process of the relationship between spirituality, knowledge and TB medication adherence. Second, mixing of methods can result in multiple inferences about spirituality, knowledge and TB medication adherence that support or compliment each other and result in better inferences. Third, mixed methods can result in a different quantitative research conclusion and qualitative research conclusion, which is acceptable. This may lead to new comprehension of the phenomenon, reexamination of the conceptual framework and assumptions underlying this study, and result in a new study for further investigation (Tashakkori \& Teddlie).

Mixing of data sources to study the same phenomenon, known as a form of triangulation (Patton, 2002), was used to strengthen this study. Triangulation allowed the researcher to confirm, cross-validate, or corroborate findings within a study (Tashakkaori $\&$ Teddlie, 1998). The collection of quantitative and qualitative data occurred concurrently during the data collection phase of the study, and the results were integrated during the data analysis phase. The qualitative data assisted in explaining the limitations of the quantitative data to gain insight into spirituality and TB medication adherence in the African American and Haitian populations. The next section discusses the quantitative research design (correlational) and the qualitative research design (phenomenological). 


\section{Correlational Research Design}

Correlational research design is a commonly used method to describe the relationship between two or more variables (Polit, Beck, \& Hungler, 2001). Being

economical, easy to implement, and having the ability to yield results in a short period of time are strengths of this design (Polit et al., 2001). Correlational research design examines the strength and direction of the relationship. The correlational phase of the study revealed no relationship between spirituality and TB medication adherence. To provide more depth, clarity and understanding, a phenomenological analysis was used to further explore the experience of spirituality.

\section{Phenomenological Research Design}

Phenomenology is a qualitative research method used to explore how human beings make sense of experience and transform experience into consciousness, both individually and as shared meaning (Patton, 2002). Phenomenology has become a popular term with a variety of meanings, such as, descriptive studies concerned with themes of perceptions, intentions, the origin of experiences and the relationship among experiences (Merriam \& Simpson, 2000). Phenomenology aims at getting a deeper understanding of the nature or meaning of our everyday experiences (Patton, 2002). The researcher collects from the interviews the perceptions of the participants' experiences and interprets their meanings (Newman \& Benz, 1998). Phenomenologists are interested in how people understand the phenomenon they experienced and how they make sense of the world and develop a worldview (Patton, 2002). This research design helped the researcher to explore the phenomenon of spirituality and its relationship with TB medication adherence. Phenomenology allowed the researcher to study a small number of 
participants' experiences of spirituality and engage in extensive and prolonged interviews to allow for patterns and meanings to emerge (Creswell, 2003).

The first step in phenomenological research is epoche (Moustakas, 1994). Epoche is seen as preparation for deriving new knowledge and an experience in itself by the researcher. Epoche involves the researcher's setting aside of prejudgments, prejudices, understandings, and knowings to allow the event, people, or things related to the phenomena to be studied and to be seen again as if for the first time. To achieve epoche, I attempted to set aside my prejudgments and understandings about spirituality and tried to see this experience from a fresh viewpoint. During the interviews I listened to the participants and did not make any judgments about their interview responses. In this study, I conducted a self interview to gain insight into my experience with spirituality, knowledge, and TB medication adherence. Epoche is followed by the second step of data collection. I described what the participants experienced and attempted to uncover, define, and analyze the elements of the phenomenon. Data were then organized into meaningful clusters.

The third step is phenomenological reduction. I examined the data, identified key phrases and themes about the phenomenon, interpreted the meanings of the themes, and developed a statement about the phenomenon. I reflected on the textual descriptions of the participants' experience. Once themes were identified, as the researcher, I conducted imaginative variation on each theme and constructed a textual description of how the participants experienced the phenomenon. Imaginative variation requires the researcher to use intuition and look at possible alternate meanings and perspectives of how the phenomenon was experienced by the participants (Moustakas, 1994). Last, synthesis of 
texture and structure is done, which is the participants' description of what happened and how the participants experienced the phenomenon. Synthesis of texture and structure requires the researcher to integrate the participants' descriptions and provide meaning of their descriptions of the phenomenon they experienced (Moustakas, 1994). Next, my autobiography, assumptions, and journal are discussed.

Researcher: Autobiography, Assumptions, Journal

Phenomenology requires the researcher to attempt to become aware of every prejudgment or assumption (Moustakas, 1994). Epoche, the first step in phenomenology, is a process that the researcher engages in to remove, or at least become aware of, prejudices, viewpoints or assumptions regarding the phenomenon being researched (Patton, 2002), so that the phenomenon can be viewed from a fresh new perspective. To achieve epoche, I attempted to dismiss my assumptions about the phenomenon before interviewing participants by documenting any prejudgments or assumptions in a journal that was used throughout this study as a data source. My autobiography and assumptions identify my biases and assumptions about spirituality and TB medication adherence.

\section{Autobiography}

I was born and raised in Miami, Florida. My education includes an undergraduate degree in nursing from Howard University in Washington, DC, and a graduate degree in public health from Florida International University, in Miami, Florida. My nursing experience includes case management, critical care, home health and public health, which has enabled me to interact with patients from various cultural, ethnic, and spiritual backgrounds. My primary experience in spirituality was a result of my growing up in the Baptist Church. My lessons in the Baptist Church focused on the importance of having a 
relationship with God. I was taught that God was in control of my life and to first seek him through prayer in every decision I made in my life. Spirituality has been an important element of my home environment. In addition, I practice spirituality in the form of prayer, meditation, and song. Every day in my home some form of spirituality was practiced. I was taught to pray to God in the morning and at bedtime. When I have to make a major decision in my life, I always pray and ask God for guidance. I believe that God is a source of knowledge and wisdom. As a result of my spirituality, I believe that I am able to deal with challenging life events and make meaning out of those events.

As an African American woman, I understand and can relate to the African American spirituality experience, especially during a health crisis. For example, whenever I or a family member has been diagnosed with an illness, I pray and ask God for a healing and to guide the physician or healthcare provider involved in my care. In the African American community, we were taught to pray for one another, especially those members who were sick, which is evident in church or other places of worship. In my professional life, I have interacted with African American and Haitian TB patients who consider themselves spiritual. Both African American and Haitian TB patients have verbalized to me a belief that God is their source of strength, is in control of their lives, and will cure their TB. I have observed African American and Haitian TB patients engaging in spiritual practices such as prayer, meditation, and songs.

Haitian TB patients have stated to me a belief that their diagnosis of TB was the result of a curse or voodoo used on them. I have interacted with Haitian TB patients who have left Miami and have traveled to Haiti to have the curse removed from them and, thus, cure their TB. Some patients returned to Miami and continued their medical 
treatment with the belief that the curse had been removed from them. Others have not returned and, therefore, did not continue with their medical treatments. My autobiography reveals my bias that I believe in a higher being or power, mainly God, and spirituality has an influence on our lives and affects our decision-making. I am open to discovering African American and Haitian TB patients' spirituality experiences in their own lives. In my professional life, I acknowledge and accept my patients' spiritual experiences, I do not preach or impose my spirituality on them, and I recognize the role of spirituality in their lives.

\section{Assumptions}

This study is based on the following assumptions:

1. We all have some form of spiritual beliefs and practices that guide out lives.

2. Spirituality is present within all of us as a transcendent force.

3. Spirituality is expressed in different forms depending on the life challenge that we encounter.

4. Spirituality has an influence on our decision making skills.

5. Spirituality can either influence or hinder learning based upon one's motivation to change.

6. Spirituality influences our health beliefs, health behaviors, and religious practices when we encounter an illness.

\section{Journal}

I kept a journal of my notes, experiences, reactions, and conversations to identify my biases, note emergent themes, and capture thoughts and new ideas related to this study (Moustakas, 1994). In addition, I recorded my observation of the participants' 
educational level. Spirituality is an important part of my life, and I am constantly engaged in some type of spiritual practice. In addition, as I continued to explore this phenomenon, I learned more about the various aspects of spirituality through reading books, journals, and interviews with TB patients. Acknowledging my biases and assumptions is important when analyzing and reporting the results for others to note the validity of the study (Gallagher, 2006). Next, the population and convenience sampling are presented.

\section{Population}

The populations of interest for this study were African American and Haitian TB patients. In 2007, 182 patients (cases) with tuberculosis were reported in Miami-Dade County (Florida Department of Health, 2008). Of these, African Americans represented 107 cases and Haitians represented 62 cases. In my job at the hospital, I have access to all TB patients and patients' records. My job responsibilities include identifying and interviewing TB patients; sending reports on TB patients to the health department as part of mandatory infectious disease reporting; and educating patients, their families, and hospital staff about TB. My job responsibilities allowed for face-to-face interviews with the participants. Face-to-face interviews were conducted once I received approval from the hospital's Institutional Review Board.

\section{Convenience Sampling}

Convenience sampling (Nieswiadomy, 2008) was used for both quantitative and qualitative data collection. All African American and Haitian TB patients at the MiamiDade County hospital or Miami-Dade County health department were eligible to participate in the study. I specifically asked Haitian patients if they were born in Haiti or 
have parents or grandparents who were born in Haiti. This sampling method involved convenience and the desire to use available participants for the study (Bernard, 2000; Levin \& Fox, 2003). TB patients diagnosed at the hospital were identified through both retrospective and forward chart reviews and lab or physician reports. Once a patient was diagnosed with TB, I contacted the patient to determine if he or she was willing to participate in the study. All suspected or confirmed TB cases are reported to the TB clinical care coordinator who reports the cases to the health department. TB patients diagnosed at the health department were identified through the health department surveillance unit staff, with which I have daily contact.

The population from which the participants were to be selected during correlational research was comprised of 33 African American and Haitian TB patients, who were all cases for 2008 and 2009. All African American and Haitian TB patients were asked to participate in the study, and written consent was obtained. A copy of the signed consent form was read to them when appropriate and given to the participants. Then, they were given the Spiritual Perspective Scale instrument to complete. If the patient did not wish to participate in the study, I thanked them for their time; I continued to contact old and new TB patients in the hospital, clinic, or by telephone.

Selecting a sample size for phenomenological research requires that all participants have experienced the phenomenon being studied and can articulate their experiences (Polit \& Beck, 2006). In qualitative research, sample sizes tend to be small (10-12 participants; Nieswiadomy, 2008). In this phenomenological study participants were interviewed once, the researcher did have a large amount of data for the majority of participants. Therefore, fewer (6-10) participants were needed in the study (Morse, 2000) 
to reach saturation, which is the point in the study where there is repetition of themes or categories (Patton, 2002). I selected a total of 16 participants: 8 African American and 8 Haitian patients. I decided to select 16 participants due to possible threats to validity such as, losing participants during the course of the study (Creswell, 2003). Participants may be lost if their final diagnosis is something other than TB, if there is a change in treatment, or if they may move out of the area. Next, instruments used in this study are described.

\section{Instruments}

The Spiritual Perspective Scale (SPS), a standardized instrument (Reed, 1986; see Appendix A), was selected to collect data because it quantifies spirituality and is easy to administer. In addition, three data sources were used. First, Medication Adherence Record (MAR; see Appendix B) was used to measure TB patients' medication adherence levels. Second, TB suspect/confirm case report (see Appendix C) provided sociodemographic data. Third, interviews were used to collect other data for this study to obtain greater insights into the influence of spirituality on African Americans' and Haitians' lives, augmenting SPS data. The SPS, MAR, TB suspect/confirm case report, and interviews are presented in this section.

\section{Spiritual Perspective Scale (SPS)}

The SPS (Reed, 1986) is a summative 10-item instrument designed to measure a person's perspective on spiritual views and activities in life that provide a sense of transcendence and connectedness to a purpose greater than the person. This instrument includes statements such as, "My spirituality is a significant part of my life" or "My spiritual views have an influence upon my life." Participants must indicate to what degree 
they agree or disagree with the statements. Items are scored on a 6-point Likert-type scale and have total scores ranging from 1.0 (no spiritual perspective) to 6.0 (greater spiritual perspective). Previous use of this instrument has demonstrated both criterion and discriminate validity estimates of $r=.71$ (Daily \& Stewart, 2007). Reliability estimates for this instrument using Cronbach's alpha were consistently greater than .90 ; inter-scale correlation ranged from .54 to .60; and all item-scale correlations were greater than .60 in previous research (Dailey \& Stewart, 2007; Gray, 2007; Reed, 1997). In this study, reliability estimates for this instrument using Cronbach's alpha was .77. I used the instrument in its original form. Dr. Reed gave me permission through email to use her instrument (see Appendix C).

\section{Medication Adherence Records (MAR)}

The MAR is a form adopted from Miami-Dade county Health Department TB unit. MAR provides a data source of TB medications and prescribed Directly Observed Therapy (DOT) visits ordered by the physician from the start of TB treatment until completion of treatment. The majority of TB patients are enrolled in the DOT program to ensure adherence to treatment. This program consists of a health department worker who delivers, observes, and records TB patients ingesting their TB medications. TB patients may be on a daily TB medication regimen, which consists of 28 prescribed visits in a month, or a three-times-a-week regimen, which consists of 12 prescribed visits a month. A patient is considered adherent if he or she attends $80 \%$ of prescribed visits (Davidson et al., 2000). Consistent with the literature, patients who consistently attended $80 \%$ of prescribed visits were considered adherent and patients who attended less than $80 \%$ of prescribed visits were considered nonadherent. At the end of 3 months, participants were 
assigned an adherence level score. The MAR is reviewed and updated weekly by the nurse or when changes in patients' medications are ordered by the physician. This record shows if the patient has adhered with his or her TB medication through the duration of his or her treatment. The MAR is not part of the patient's medical record. This is a tool the $\mathrm{TB}$ clinical care coordinator and physician maintains and uses in the clinic to monitor TB patients' medication adherence.

\section{TB Suspect/Confirm Case Report}

The TB suspect/confirm case report is a health department form that consists of sociodemographic data such as name, race, ethnicity, date of birth, place of birth, employment, and marital status. In addition, this form contains medical information such as symptoms, bacteriology, site of the disease, and medication start date and regimen. The TB clinical care coordinator completes and submits this form to the health department for all TB patients.

\section{Interviews}

Interviews allow the researcher both to find out what is on a participant's mind and to gather his/her stories (Patton, 2002). Interviews conducted in person ensure a faceto-face encounter with research participants and allow the researcher an opportunity to elicit additional information (Merriam \& Simpson, 2003). Interviews are useful in gathering data when the topic to be explored is complex and emotionally sensitive, providing historical information, and allowing the researcher to have control over the questions to be asked. As with any method of gathering data, however, there are disadvantages to semi-structured interviews, which were used in this study. For example, the presence of the researcher may cause participants to present biased responses. To 
reduce the participant's biased response, I developed a conversation with the interviewees to gather information about their experience (Patton, 2002) since participants may not all have the same level of perception and ability to articulate their response to interview questions (Creswell, 2003).

Based on the research questions, I developed a semi-structured interview guide (see Appendix D). The semi-structured interview guide includes a set of questions to ask all participants and allows for impromptu questions to follow up on leads that emerge during the interview (Nieswiademy, 2008; Polit \& Beck, 2006; Poutin, 2000). Both closed-ended and open-ended questions were included in semi-structured interviews. Closed-ended questions required participants to choose from given alternatives, whereas, open-ended questions allowed participants to answer in their own words (Nieswiadomy, 2008). Since TB medications and treatment regimens remained the same, seven new TB cases and nine former TB cases agreed to be interviewed. Due to the issue of attrition, sixteen patients were selected and interviewed, which resulted in a sample size of 16 participants at the end of the qualitative data collection phase. Participants were interviewed at the hospital and outpatient clinic. Interviewees' responses were written and audiotape recorded by the researcher.

\section{Procedures}

Procedures, involving the pilot study, gaining access, use of an interpreter, data collection, and data analysis are presented in this section. 


\section{Pilot Study}

A pilot study was conducted with eight patients to determine if the directions for the SPS were clear and to get an estimate of the amount of time to complete the instrument. The SPS completion time for English-speaking participants ranged from 5 to 10 minutes. For Creole-speaking and illiterate participants who required assistance completing the SPS, completion time ranged from 10 to 15 minutes. In addition, interviews were scheduled with both an African American and a Haitian patient to assess the questions for clarity and appropriateness, and to obtain an estimate of the time needed to complete the interviews. The African American participant completed the SPS in 10 minutes and the interview in 30 minutes. The Haitian participant completed the SPS in 10 minutes and did not return to the clinic to complete the interview.

\section{Gaining Access}

Permission to conduct this study and collect data was obtained from the Institutional Review Boards (IRB) of the county hospital (see Appendix F), Florida International University (see Appendix G), the State of Florida Department of Health (see Appendix H), and University of Miami (see Appendix I). The State of Florida Department IRB application process required a letter of support from the Director of Miami-Dade County Department of Health. The process to obtain the letter of support from the Director of Miami-Dade county Health Department required clearance by the chief legal counsel office. I submitted my proposal to the legal office and met with the chief legal counsel, medical director of TB Control, and the medical director of Miami Dade County Department of Health. This process took five months to complete. 
Eventually, I was granted a letter of support from the Director of Miami-Dade County Health Department, which I submitted to the State of Florida Department of Health IRB.

\section{Use of an Interpreter}

A large percentage of the TB patients admitted to the county hospital are Haitian, and many do not speak English or read literature written in English or Creole. The consent forms were translated into Creole and back translated into English by two Creole-speaking healthcare workers. A trained interpreter assisted the researcher in explaining the purpose of the study; obtaining consent to participate in the study; and verbally, if appropriate, administering the SPS instrument at the hospital, out patient clinic, and health department. Gender, racial identity, social class, and shared experiences may have influenced the interview process and affected the willingness of the patients to participate in the research. Problems of insider versus outsider status may have also been an issue during the interview with Haitian TB patients (Kapborg \& Bertero, 2002;

Pitchforth \& van Teijlingen, 2005); a Haitian Creole-speaking healthcare worker assisted with recruitment of TB patients and served as an interpreter while I conducted interviews with the Creole-speaking TB patients. The trained interpreter signed a statement of confidentiality (see Appendix E).

\section{Data Collection}

Once a TB diagnosis was confirmed, all participants received verbal and written explanations about their TB diagnoses and TB medication regimens. Data were collected during minimally 30 -minute to one-hour person-to-person interviews through the SPS and a review of all participants' TB Suspect/Confirmed TB Case Reports and MARs over a 3-month period in 2009. Demographic data was obtained from the Suspect/Confirmed 
TB Case Report. The SPS was administered in the hospital, health department, and outpatient clinic. Study participants in the outpatient clinic were given the instrument while in the waiting areas prior to their physician visit. The SPS was placed in individual envelopes with labels identifying the location and research participant by number and that number was placed on the SPS. Completed SPSs were placed in the data collection envelopes, sealed by the participants, and returned to me.

I reviewed each participant's MAR during the hospital admission and at the end of three months to determine if the patient was adherent with treatment. A retrospective chart review of previous TB cases was done to select participants to complete the SPS. I contacted the patients by telephone and explained my study. If the participant agreed to participate, I met the participants at their next outpatient clinic visit and they completed the SPS. Toward the middle of the data collection process, the outpatient TB clinic at the county hospital was closed for one month due to a burst water pipe. This development delayed recruitment of prospective participants.

Concurrent procedures allow the researcher to combine quantitative and qualitative data to provide a comprehensive analysis of the research problem (Creswell, 2003). The researcher collected both types of data at the same time and then integrated both during data analysis. Quantitative and qualitative data were entered into a final database. To summarize, the following steps were followed to collect both quantitative and qualitative data:

Step 1. Researcher identified the TB patient and introduced herself to the patient. 
Step 2. During hospital stay or at the first outpatient clinic visit, the researcher provided TB education about signs/symptoms, transmission, prevention, medications to the patient, and the importance of medication adherence.

Step 3. Researcher explained the purpose of the study and obtained patient's consent. Step 4. Researcher administered the SPS and gave patient an envelope to insert the completed instrument.

Step 5. Researcher scheduled a time and met the patient during the hospital stay or at outpatient clinic one month after discharge from the hospital and conducted the interview.

Step 6. Researcher reviewed each patient's MAR at the start of treatment and one month after initiation of TB medications. The next section presents a discussion on the quantitative and qualitative data analysis methods used in this study.

\section{Data Analysis}

All quantitative data were analyzed using the Statistical Package for Social Sciences Graduate Pack 15.0 (SPSS) software program. To answer the primary research, I computed a Pearson's product- moment correlation coefficient on the SPS and MAR data. A Pearson's product- moment correlation is commonly used in research when both sets of data are at the interval or ratio level (Nieswiadomy, 2008). However, when one of the variables is dichotomous, Point biserial correlation is used in research (Polit, Beck \& Hungler, 2001).

All qualitative data were analyzed, based on Patton's (2002) phenomenological analysis processes. The steps in Moustakas's Transcendental phenomenological model (1994) were followed after each participant was interviewed: 
1. Epoche.

2. Each participant transcripts on the influence of spirituality and TB medication was reviewed to locate key phrases and statement that were relevant to the phenomenon.

3. I interpreted the meanings of the phrases and obtained participants' interpretation of the phrases.

4. I inspected` the meanings for any essential recurring statements or words.

5. I wrote a tentative definition about the reoccurring themes identified.

6. Data were spread out for examination and then organized into meaningful clusters.

7. Irrelevant, repetitive, and overlapping data were eliminated.

8. I identified invariant themes within the data; then, I reflected and conducted an imaginative variation on each theme. This allowed me to develop enhanced meanings and relationships of the themes.

9. The meanings and themes were synthesized into a textural description of the participant's experience. Textual description provides an idea of what the participant experienced. This process is known as phenomenological reduction.

10. I reviewed the textual description of the participant's experience and through imaginative variation developed a deeper meaning and structural description of the participant's experience.

11. Member checking was done with the participants at their next clinic visit. I reviewed my description of their experience with the participants to verify 
my interpretation of their experience.

12. In the last step, I integrated the textual and structural descriptions and provided a description of meanings and essences of the participant's experience.

These steps were conducted after each participant's interview was transcribed and before the next interview was done. Both quantitative and qualitative data were analyzed simultaneously during the data collection phase of the study. Integrity measures, limitations of the study, and data management are provided next, followed by a summary.

\section{Integrity Measures}

Integrity measures involved content validity, concurrent triangulation during data collection, and triangulation during data analysis.

\section{Content Validity}

Content validity is a nonstatistical method to reflect that SPS measures what it was designed to measure. To enhance content validity, I had four expert peers (two registered nurses, one social worker, and a physician) with expertise in the healthcare field review the SPS instrument and interview guide to evaluate appropriateness and clarity for this study. Content validity was supported by my expert peers and pilot study participants; all were in agreement about the appropriateness and clarity of both the SPS and interview guide. Therefore, no changes were made to the SPS or interview guide.

\section{Concurrent Triangulation during Data Collection}

Concurrent triangulation involves collecting quantitative and qualitative data at the same time. This strategy was selected as a means to offset the weakness in one method with the strength of the other (Creswell, 2003). By synthesizing different 
methods, a richer and better understanding of the study will emerge (Teddlie \& Tashakkori, 2009). During the quantitative phase of the study, possible threats to the internal validity included history, selection bias, volunteer bias, maturation, and mortality (Polit et al., 2001). Selection bias refers to pre-existing differences between groups under study (Polit \& Beck, 2006). Maturation occurs when changes take place within participants due to the passage of time (Nieswiadomy, 2008). For example, the participant may experience dementia or forgetfulness. Mortality refers to the loss of participants from different groups (Polit \& Beck, 2006). Once the physician diagnoses the patient with TB, the patient is considered a TB suspect until confirmation is made by further testing, such as Polymerase Chain Reaction DNA test or the final culture grows TB. Therefore, some participants were lost during the course of this study due to delay in diagnosis, change in diagnosis, drop out, or death. Three participants were lost during the course of the study due to a change in diagnosis. One Haitian male participant refused to complete the interview.

\section{Triangulation during Data Analysis}

During data analysis, triangulation provided different ways of looking at the same phenomenon and added to the study's credibility by strengthening confidence in conclusions drawn from quantitative and qualitative data collection methods. The following types of triangulation contributed to verification and validation of qualitative analysis: (a) method triangulation, (b) triangulation of data sources, (c) analyst triangulation (Patton, 2002). First, method triangulation involved collecting data from both quantitative and qualitative methods. This method allowed me to discover compatibility and to determine the degree and nature of the compatibility between the 
two methods. Areas of convergence between the two methods increased confidence in the findings that showed no relationship between spirituality and TB medication adherence. Second, triangulation of data sources required that I compare and cross-check the consistency of information such as what the participants said about the same experience over a three month time period. I compared observations from participants' interviews and compared this information with medical documents that confirmed what the participant reported. Third, analyst triangulation involved two or more persons independently analyzing the qualitative data and providing feedback. The peer review group, which consisted of four doctoral candidates, reviewed my data and provided feedback. My dissertation advisor and my dissertation committee also provided feedback on my study data. Based on the feedback I received, changes were made such as additional charts were added and reanalyze participants' interview transcripts to identify themes to make my data analysis more meaningful to my study.

\section{Limitations of the Study}

Several limitations were encountered during the course of this study. First, this group of participants included patients with other medical conditions and patients outside of the hospital. Second, control of researcher's access to health department participants was not possible; therefore, that was a threat to the internal validity associated with this study. Third, 8 African American and 9 Haitian TB patients refused to be interviewed for this study. Fourth, mortality occurred in that 3 participants were dropped from the study because their final diagnoses culture grew mycobacterium other than tuberculosis (CDC, 2008). A fifth limitation maturation occurred, several participants displayed maturation in the form of forgetfulness during the interviews. This may be due to some of the 
participants having completed TB treatment. Last, from the design to implementation of this study there was a decline in the number of TB cases among African American and Haitians in Miami-Dade County, which limited the number of potential participants for this study. However, there has been an increase in the number of TB cases in Hispanics (CDC, 2009). Using convenience sampling did not allow for generalization to the larger population. The limitation posed by using convenience sampling and the limited number of TB cases can be addressed in future large scale studies over a longer time period (Polit et al., 2001).

\section{Data Management}

Data was managed according to the guidelines in the Florida International University Regulations for Thesis and Dissertation Preparation Manual (2007). All collected envelopes of SPS were kept in a locked file cabinet in my office. Completed instruments, interview recordings, and transcripts were secured in a locked file cabinet in my office. MARs are kept in a file box in a locked medication room in the outpatient clinic. All interviews and SPS had the location where the interview and SPS were administered noted on both forms (hospital, clinic, or telephone). All study data will be kept for 3 years after completion of the study (Florida International University Regulations for Thesis and Dissertation Preparation Manual, 2007).

\section{Summary}

Using a mixed method research design, 8 African American and 8 Haitian TB patients were interviewed about their spirituality and how spirituality influenced TB medication adherence. The SPS instrument was administered to 33 TB patients in the 
hospital, outpatient clinic, and at the county health department. The findings of this mixed method study are presented in chapters 4 and 5 . 


\section{CHAPTER IV \\ QUANTITATIVE DATA ANALYSIS}

This chapter presents results of the study that include descriptive statistics, chisquare and crosstab analyses, intercorrelations, and one-way ANOVAs (Levin \& Fox, 2003; Nieswidomy, 2008; Polit \& Beck, 2006). This chapter concludes with a summary of the results.

\section{Descriptive Statistics}

Descriptive statistics using frequency counts were used to present the study data (Nieswidomy, 2008; Polit \& Beck, 2006). There were a total of 33 participants in this mixed-methods study. A total of 16 African Americans and 17 Haitians completed the SPS instrument. Of this total, eight African American and eight Haitian TB patients were interviewed to gain insight into the relationship between their spirituality and TB medication adherence. Seventeen participants refused to participate in the qualitative interviews. Three participants were eliminated due to their final culture growing a mycobacterium other than TB. One Haitian female participant traveled to Haiti on December 31, 2009 and, unfortunately, was in Haiti during the January 12, 2010 earthquake. She did call her social worker and informed her that she survived the earthquake and plans to return to Miami as soon as she could leave Haiti. In addition, she reported that she found a free clinic that provided her TB medications.

Study data were entered from Excel into the Statistical Package of the Social Sciences (SPSS) version 15.0. Tests were run in SPSS to provide the descriptive statistics for the participants in this study 


\section{Chi-square $\left(\chi^{2}\right)$ Analyses}

Participants' background data includes sex, marital status, ethnicity, language adherence, and age. A total of 14 women (42\%) and 19 men (57\%) participated in this study. The participants' ages ranged from 24 to 60 , with a mean age of $34.0(S D=.72)$. Four (12\%) of the participants were married. Twenty three (67\%) of the participants spoke English as their primary language. Twenty eight (84\%) of the participants were adherent with their TB medications. Table 2 reports the chi-square analyses of the study variables.

Table 2

Chi-square Analyses of Study Variables

\begin{tabular}{llll} 
Variable & $\chi^{2}$ & $d f$ & $p$ \\
\hline Sex & .273 & 1 & .602 \\
Marital Status & 18.939 & 1 & .000 \\
Ethnicity & .030 & 1 & .862 \\
Language & 5.121 & 1 & .024 \\
Adherence & 39.455 & 2 & .000 \\
Age & 4.909 & 2 & .086 \\
\hline
\end{tabular}

The chi-square analyses indicated that the participants in this research were more likely to be married, English speaking, and adherent. 


\section{Crosstab Analyses}

To further examine possible linkages between the study variables, crosstab analyses were conducted. Table 3 a provides results of the Crosstab analyses that suggest there were no differences in language, adherence and ethnicity by sex.

Table 3

Crosstab Analyses of Sex, Language, and Ethnicity

\begin{tabular}{lccc}
\hline Variable & $\chi^{2}$ & $d f$ & $p$ \\
\hline Sex * Language & 1.22 & 1 & .269 \\
Sex * Adherence & 4.911 & 1 & .086 \\
Sex * Ethnicity & .036 & 1 & .849 \\
\hline
\end{tabular}

The results of the Crosstab analyses presented in Table 4 suggest there were no differences in language, adherence and ethnicity by marital status.

Table 4

Crosstab Analyses of Marital Status, Language, Adherence and Ethnicity

\begin{tabular}{lccc} 
Variable & $\chi^{2}$ & $d f$ & $p$ \\
\hline Marital status * Language & .836 & 1 & .361 \\
Marital status * Adherence & 1.595 & 1 & .450 \\
Marital status * Ethnicity & 1.005 & 1 & .316
\end{tabular}


According to the Crosstab results presented in Table 5, there was greater participation of English-speaking African Americans rather than Creole-speaking Haitians in this study. There was no difference in adherence by ethnicity as well. Table 5

Crosstab Analyses among Ethnicity, Language, and Adherence

\begin{tabular}{lccc}
\hline Variable & $\chi^{2}$ & $d f$ & $p$ \\
\hline Ethnicity * Language & 13.50 & 1 & .000 \\
Ethnicity * Adherence & 2.877 & 2 & .237 \\
\hline
\end{tabular}

Intercorrelations

Correlation analyses suggest that Haitians were more likely to be Creole speaking and African Americans English speaking (see Table 6). No other significant relationships were found among the background variables. When examining the relationship between SPS and the background variables (sex, marital status, age, ethnicity, and language), no statistically significant relationships were found; likewise, there was no relationship between SPS and adherence. It is important to note, however, that the direction of relationship between spirituality as measured by the SPS and adherence was in the direction predicted, that is, greater adherence was associated with greater spirituality. Overall, I failed to reject the null hypothesis in that there was not a statistically significant relationship between spirituality and TB medication adherence $(p=.208)$ among African Americans and Haitians. 
Table 6

Intercorrelations among Research Variables

\begin{tabular}{llllllll}
\hline Variable & 1 & 2 & 3 & 4 & 5 & 6 & 7 \\
\hline 1. Sex & 1.00 & & & & & & \\
2. Marital Status & .034 & 1.00 & & & & & \\
3. Age & -.160 & -.341 & 1.00 & & & & \\
4. Ethnicity & .033 & .175 & .121 & 1.00 & & \\
5. Language & .193 & .159 & -.124 & $.640 * *$ & 1.00 & & \\
6. SPS Total & .094 & .119 & -.146 & .114 & .181 & 1.00 & \\
7. Adherence & -.071 & -.210 & .195 & .076 & -.051 & .208 & 1.00 \\
\hline
\end{tabular}
Note. $* * p<.01$

\section{One-Way ANOVAs}

To explore the data further, a series of one-way ANOVAs were run to test for differences among the variables. First, one-way ANOVAs were run to examine possible group differences between the dependent variable spirituality, and independent variables sex, marital status, age category ( 3 categories; $24-34$ years of age $=1 ; 35-45=2 ;>45=$ 3), ethnicity, and language. The results did not reveal statistically significant differences among any of the variables. The $F$ s ranged from .122 to 1.05 , with the $p$ values ranging from .304 to $.886(p<.05$ is conventional level of statistical significance). Thus, there were not significant group differences in spirituality by sex, marital status, age, ethnicity, and language.

Second, the researcher also conducted one-way ANOVAs to examine possible group differences between dependent variable adherence, and independent variables sex, 
marital status, age category ( 3 ; categories; $24-34$ years of age $=1 ; 35-45=2 ;>45=3$ ), ethnicity, and language. Again, the results did not reveal statistically significant differences among any of the variables. The $F$ s values ranged from .082 to 1.43 , with the $p$ values ranging from .240 to .883 . Thus, there were not significant group differences in adherence by sex, marital status, age, ethnicity, and language.

Summary

This chapter presented the quantitative results of a mixed-method study. The results fail to reject the null hypothesis; there was no relationship between spirituality and TB medication adherence among African Americans and Haitians. Chi-square analysis revealed the participants were more likely to be married, English speaking, and adherent with their TB medications. 


\section{CHAPTER V}

\section{QUALITATIVE ANALYSIS}

This chapter presents the qualitative analysis in three sections: (a) description of the sample, (b) thematic analysis, and (c) summary.

\section{Description of the Sample}

A total of 33 participants completed the Spiritual Perspective Scale (SPS). The participants' ages ranged from 24-60. Sixteen participants, eight African Americans and eight Haitians, were interviewed. Of the sixteen participants interviewed, $21 \%$ were female $(n=7)$ and $27 \%(n=9)$ were male. A total of 17 participants refused to participate in the interviews. One participant's interview ended early due to the participant becoming agitated and angry during the interview. Of the 33 study participants, 17 were currently on treatment and 16 completed treatment within the last year (see Table 7). The names of the participants in this study are pseudonyms.

The majority of the sample was recruited in the outpatient TB clinic at a large urban hospital. Due to State of Florida Department of Health IRB rules, the health department surveillance analyst was responsible for recruitment of health department patients. Once the surveillance analyst identified the potential participant and obtained consent, I was notified and then I met the participant at the health department clinic.

The table results show that not all participants who completed the SPS agreed to be interviewed. The majority of participants interviewed were male and over age 40 . Most of the participants interviewed were TB patients who had completed their TB treatment. 
Table 7

Description of Sample

\begin{tabular}{|c|c|c|}
\hline Variables & Frequencies & Percent \\
\hline \multicolumn{3}{|c|}{ Completed Spiritual Perspective Scale } \\
\hline Yes & 33 & 100.0 \\
\hline No & 0 & 0.0 \\
\hline \multicolumn{3}{|l|}{ Age } \\
\hline $20-30$ & 2 & 6.1 \\
\hline $31-40$ & 7 & 21.2 \\
\hline Over 40 & 24 & 72.7 \\
\hline \multicolumn{3}{|l|}{ Ethnicity } \\
\hline African American & 8 & 24.2 \\
\hline Haitian & 8 & 24.2 \\
\hline \multicolumn{3}{|l|}{ Sex } \\
\hline Male & 9 & 57.6 \\
\hline Female & 7 & 42.4 \\
\hline \multicolumn{3}{|l|}{ Interviewed } \\
\hline Yes & 16 & 48.5 \\
\hline No & 17 & 51.5 \\
\hline \multicolumn{3}{|l|}{ TB medication } \\
\hline Yes & 17 & 51.5 \\
\hline No (Completed) & 16 & 48.5 \\
\hline
\end{tabular}




\section{Thematic Analysis}

Thematic analysis was performed to analyze participants' responses to the semistructured interviews. The interview questions were grouped into three sections relating to (a) the role of spirituality, (b) knowledge of TB, and (c) medication adherence. First, the data from each interview were looked at to identify words or phrases relevant to the research questions. Thematic analysis was then conducted to identify themes occurring within and across each group. Data from each group were analyzed separately, and common themes that emerged in each group were identified and compared. Data were examined within each ethnic group and then across each group to determine similarities and differences between the groups. During the data analysis phase, I met with my major professor both to discuss the data and to identify themes relevant to the phenomenon in this study.

This section presents the qualitative data and thematic analysis for African Americans, Haitians, and across groups. Verbatim responses are provided.

\section{African Americans}

Four themes emerged from the thematic analysis of African American participants' data: (a) God is in control, (b) stigmatization of TB, (c) lack of knowledge, and (d) fear of death.

\section{God is in Control}

Spirituality as a relationship with a transcendent force that brings meaning and purpose to one's existence and affects the way in which one operates in the world (Armstrong, 1996; Mattis, 2005). As mentioned in Chapter one, African Americans believe in a strong, intimate relationship with God and the ability to overcome health 
limitations through the power of prayer (King et al., 2005). Spirituality and religion have been used interchangeably to describe concepts related to God or a higher power, things sacred or divine, or a heightened level of human consciousness (Utsey et al., 2007). When asked what spirituality meant to them, five participants made reference to a belief in God, and three participants referred to a belief in the Lord. Joe, Mark, Jeffrey, Joan, John, and Pat described spirituality as a belief in God or a higher power. In addition to describing a belief, the participants included spiritual behavior in the form of prayer. When asked what spirituality meant to him, Joe explained how he turned to God in fear:

Because I believe not only from what I was taught and told but in a lot of personal experiences in my life in the last 15, 20 years. And you know being close to getting it and coming up out of it, praying and praying in the hospital when I wouldn't pray before and you know and come through it when I was told and know that I wasn't supposed to. I prayed and realized that there is a God and there is a higher power. It's a spiritual awakening so I still don't pray as much as I should but I pray. (Joe, Lines 22-27)

The participants' responses included beliefs, behaviors, life experiences, and the ability to surrender to God and not worry about their illnesses. Spirituality expressed as a belief in God or a higher power served as a coping skill and social support, fostered feelings of optimism and hope, promoted healthy behavior, reduced feelings of depression and anxiety, and encouraged relaxation (Newlin, Knafl, \& Melkus, 2002). Joe's response shows how spirituality served as a resource that helped him cope with having TB, and the experience of being hospitalized and engaging in prayer increased his belief in God and reduced his anxiety. Mark's response is another example of belief in God and surrender to God as a source for coping with his illness. To Mark:

Spirituality means believing in God. Ah the higher power, something that you can't really see and something that controls the world in other words, to me I believe in God anyway so, that's the only person that keeps me going. He got the 
last say so, not the doctors, not nobody on earth. God got the last say so about everything. (Mark, Lines 2-6)

Joe and Mark explained what spirituality meant to them; both mentioned a belief in God and surrender to God that served as a coping mechanism.

In addition to describing spirituality as a belief in God and source of coping, Joe Joan, John, and Mark described life experiences that strengthened their belief in God. Spirituality served as a way for the participants to interpret their life experiences based on their belief in God (Johnson et al., 2005). John gave an example of a time when he reached a low point in his life:

I know there is a higher power. I believe in God. I was out there smoking, smoking crack and using the pipe behind people, I was sick, so I had to turn my life over to a higher power. (John, Lines 18-21)

Spirituality involves faith or a willingness to believe, a search for purpose in life, a sense of connection with others or a transcendence of self, resulting in a sense of inner peace and well-being (Delgado, 2005; Mattis, 2005). John's response shows how his spirituality, based on his belief and willingness to surrender and have faith in a higher power, helped him to cope with his drug habit and illness.

Joan appeared to have a difficult time explaining what spirituality meant to her. Joan's response included a reference to the Lord, instead of God, as the higher being. In the African American community, the name Lord and God are used interchangeably. She gave an example of how spirituality served as a source for coping with life. "I didn't give up on myself because I know that the Lord was able to move this" (Joan, Line 33). Later during the interview, I asked Joan to tell me what spirituality meant to her, Joan explained: 
It was good cause you know you have that willing, that power, that belief, that you're gonna overcome that situation. That's what I had and I came out to be a graduate from TB. No more TB. (Joan, Lines 82-84)

Joan's response described the emotional aspect of spirituality as well as how spirituality

was used as a positive resource that helped her to cope with a challenging life experiences and TB:

I feel joy. I feel good. I feel ah, uplifting. It takes off a burden, it takes off the weight, 'cause you go through things from day to day. You go through a lot of things but then when I go to church and I get in my Word, it uplifted my spirit to give me hope that everything gonna be alright. You know how sometimes how it gets in your mind, well you say oh, you start focusing back on little things that's going on in your body and I tell ya I just block it out. And every day I just go on, keep calling on the name of the Lord and do positive things and I get good reports when I go see my doctor. (Joan, Lines 60-64)

This shows the importance of spiritual beliefs and practices, such as prayer, for coping with illness in the African American community (Johnson et al., 2005). Jeffrey's response, like Joan's, described spirituality as a belief that influenced his behavior and served as a positive force that guided his life:

Being a certain way, acting and living a certain way because you believe a certain way. It makes you act different. It keeps you grounded and you don't do certain things. Spirituality means good things to me. It makes life worth living and helps keep me straight. It keeps me out of trouble. It something I try to practice every day. (Jeffrey, Lines 2-8)

Jeffrey's response revealed that his faith in God was a source of protection after being diagnosed with TB. Jeffrey described the positive role of spirituality in his life and how it gave meaning to his life. According to Jeffrey, "God is my protector and he gives me strength" (Jeffrey, Line 11). Joe's response described the role of spirituality in his life as he encountered a life experience:

But after I came through it, I went through it and came out of it and realized what I endured and where I'm headed opposed to where I'm supposed to be that was a 
spiritual awakening for me so it kind of strengthened my belief of spirituality. (Joe, Lines 35-39)

Pat used the name Lord and God interchangeably in the same statement to describe her definition of spirituality, "I believe in the Lord and I feel like this here; you know, what God got for me I want all he took from me, I thank Him so I don't complain about nothing" (Pat, Lines 4-6). Pat's response described the emotional aspect of spirituality as a resource she used to cope with having TB. Pat said, "And I keep my joy, I don't let nobody take my joy away from me" (Pat, Line 33). Pat's response shows how African Americans' external and internal coping resources are based on a strong spiritual foundation (Utsey et al., 2007).

\section{Stigmatization of TB}

Stigma is part of a system of beliefs about illness and disease that is often grounded in social inequalities (Castro \& Farmer, 2005). In many cultures, the social stigma of TB and discrimination by healthcare professionals contribute to abandonment of treatment and lengthy delays in seeking professional care (Ilongo, 1994; Rubel \& Garro, 1992). Coreil (2001) reported that TB patients are fearful of disclosing their illness to others due to the stigma that is attached to the disease. In the African American community, TB is considered a dirty and mysterious disease that affects bad people. As a result, TB patients often experience stigmatization from family and friends (Jenkins, 1966; Kelly, 2000). The responses of two participants, Pat and Jeffrey, described their experience of being stigmatized as a result of their TB diagnosis. Pat revealed how she experienced stigmatization from a family member:

Now my sister, she was like that. She was scared like I'm gone give y'all something or this, that, and the other, and stuff like that there. Cause when I left, I 
had left to go to my mom's funeral. Where Pat gone stay at and my sister, I heard my sister say, oh she can't stay with me cause she got full blown tuberculosis and my brother say, you think these people would let my sister, let your sister out here and knowing that she got full blown and she gone hurt somebody? (Pat, Lines 219-225)

Reactions, such as fear of contracting TB and avoidance from family members, contributed to the patients' fear of disclosure of TB. Fear of disclosure interfered with the TB patients' learning or obtaining knowledge about TB. Jeffrey explained, "I do not read TB booklets. I don't want people to know my business. It ain't nobody business but my own. I don't want any TB books or papers that say I have TB" (Jeffrey, Line 44). Participants' concerns about perceived stigma and misinformation regarding TB in the community interfered with participants knowledge acquisition.

\section{Lack of Knowledge}

'Knowledge is defined as a human beings' understanding about reality through mental correspondence, personal experiences, and emotional affection with outside objects and situations" (Yang, 2003, p. 108). Six of the participants stated their lack of knowledge about TB. Two of the women participants, Lisa and Kathy, verbalized knowledge about TB signs, symptoms, and transmission mode. John, Joe, and Jeffrey described their experience learning about TB. "The first time I learned about it was when I contracted it. That's when I first learned about it. And I didn't know nothing about TB" (John, Line 42). Joe said, "I did not understand what I was taught during the course of my TB treatment until I contracted another disease that affects patients with compromised immune systems" (Joe, Line 149).

One of the women participants, Pat, said, "I never knowed nothing about it until it happened to me" (Pat, Line 5). Statements made by the participants indicated their lack of 
explicit knowledge (Yang, 2003) about the disease. Lack of knowledge about TB may have led to the denial of symptoms, which leads to a delay in seeking treatment and medication adherence. Jeffrey explained his reason for lack of knowledge of TB, "So sometimes I don't believe that I have TB. Doctors do make mistakes" (Jeffrey, Line 32). Jeffrey's response is an example of how denial of TB disease may interfere with learning about TB and medication adherence.

Fear of Death

Acceptance or denial of their illness may influence a patient's decision to seek medical care and take medications (Adams, Pill, \& James, 1997). Belief in the effectiveness of TB medication was evident in all participants' responses to a question regarding medication adherence. The participants verbalized and understood that in order to cure TB they had to be adherent with their medication. For example, Joe stated that he lacked knowledge about TB, but he verbalized an understanding of the importance of medication adherence. Joe said, "I believe the medications will work and make me feel better (Joe, Line 63). Joan's response is an example of what motivated her to be adherent with her TB medications, "I wanna live to get better, and better, and better. And as I continue to take my medicine, I feel myself getting better and better and better" (Joan, Line 178). The will to live and hear positive reinforcement from her healthcare provider contributed to Joan's TB medication adherence.

Joe expressed the will to be healthy and live as his motivation to be adherent with his TB medication. Joe responded to the question about medication adherence:

Now I know how it feel to keep, to keep, um, to keep healthy and keep you from getting out of control. If you wanna live again, if you wanna live and stay healthy 
and live longer, you have to trust me, it, it, it, ain't nothing you like, (laughing), I ain't even a pill liker to tell you the truth. (Joe, Line 214)

Three types of behaviors have been identified as barriers to medication adherence: (a) denial, (b) avoidance, and (c) depression (Walsh, Hagan, \& Gamsu, 2000). Emotions, along with acceptance of the disease, may enhance or interfere with TB medication adherence. Pat's response, like Joe's, described her emotional experience of being diagnosed with TB and how she accepted her diagnosis and knew that in order to live she had to be adherent with her medication:

It was a hurting thing but in order for me to live I'd rather take it and feel good about taking it to live than be feeling bad about it. 'Cause it's not something you could get rid of right then and there, so, you might as well, you know I feel like I gotta deal with it so I might as well be happy take my medicine and do what I have to do. (Pat, Line 8)

During the course of her TB treatment, Pat stated the importance of medication adherence. Pat said, "The medication works good, medication works if you work with it" (Pat, Line 86). Joe and Joan stated how the spiritual practice of prayer as well as their belief in the effectiveness of the medications contributed to their living with TB. Joe explained, "I just asked God to hold me in his hands and you know, let me live and if I could add waking up every day and I could take my medicine and I could do right, I'd live longer" (Joe, Line 75).

Joan's response revealed how her desire to live her faith in God contributed to her TB medication adherence. Joan explained, "No, I just myself to continue taking my medicine and keep faith in God he'll move your situation, to get well and live. I want to live until God calls me home" (Joan, Line 184). In the African American community, the quote "until God calls me home" means death. Four of the participants directly stated the 
fear of death as motivation for being adherent with their TB medications. Jeffrey, a 26year-old single man, verbalized his fear of death, "I don't want to die. I am young and I want to live. This is no joke. I don't want to be sick again and end up in the hospital, so I take my medications that the doctor ordered" (Jeffrey, Line 62). Whereas, Mark verbalized his acceptance of his mortality and he stated that taking his TB medications would help extend his life. Mark explained why he has been adherent with his TB medications:

I just got to do the right thing and take my medicines, stay healthy, and do like I'm supposed to do and I won't have to worry about rushing to die and all that. I don't worry about that, Um'ma [I'm going to] die anyway. I don't worry about that stuff. (Mark, Line 82)

Joan described how she convinced herself to realize how important medication adherence was for her to get through her treatment regimen:

So do you wanna live or die? So that was the part where I had to go head and take it. It was a journey, it was a lot of pills but hey we got, you'll get through it. You'll get through it, throughout the day you'll get through it because you want to see results. And the medicine does work. (Joan, Line 139)

Pat verbalized the positive aspect of medication adherence, based on her experience before she was actually diagnosed with TB:

Why not take the medicine if it's gone help me? So this, having this experience, um, it made you more motivated to do positive thing. Before I knowed I had it, it was like I was little, losing weight, feeling bad, you know, so, when I found out what I had, I kind of was hurt but then I had to look at life, if I take my medicine it's gone help me, if I don't take it, it's not gone help me. And I could end up losing my life, so you know. (Pat, Line 155)

The African American participants' responses show that satisfaction with interpersonal care and the perception of TB medication effectiveness motivated them to be adherent 
with their medications (McDonnell, Turner, \& Weaver, 2001). The next section presents the themes that emerged from Haitian participants' data.

\section{Haitians}

The Haitian participants described a view of spirituality similar to that of the African American participants with the exception of the practice of Voodoo. All Haitian participants described their spirituality as a belief in God. Four themes emerged from the thematic analysis of Haitian data: (a) my God is bigger than Voodoo, (b) stigmatization of TB, (c) lack of knowledge, and (d) fear of death.

\section{My God is Bigger than Voodoo}

In Haiti, the two main religions that are practiced are Voodoo and Catholicism.

However, Haitians in the United States are active in several mainstream religious groups, and the church is a source of support (Etienne \& Pavlovich-Danis, 2010). Haitian participants did verbalize the existence of Voodoo practices; however, none of them stated that they practiced Voodoo. They emphasized that Voodoo is commonly practiced in Haiti, but not in Miami, Florida. Jean's response described his faith in God as opposed to Voodoo:

Everything that is possible for him, only God can do for him. Ok, I don't believe in Voodoo but I knows that Voodoo exist because in Haiti that's mostly what they practice. But I believe in God so I don't care about Voodoo. (Jean, Lines 18-23)

Joel stated that his father was a Voodoo priest and that he had knowledge of this practice:

Voodoo that's a thing like for generations. So you know my daddy was a Voodoo priest. And that's where the spirit would come from. You could see the spirit in your dream and it can come on you like it's on you see something come inside you it can make you talk and see something and make you do something that you never did before. Some people practice it for the good things. (Joel, Lines 64-69) 
Based on Joel's description of his dream, it appeared that he may have practiced Voodoo or been affected by it in some way. However, when asked what spirituality meant to him, he responded:

I believe in God because God do a lot of things for me because of the way, you know, I was before, I know God is gonna do more for me. Before I was very bad, bad, bad, bad, but the situation changed and now I'm fine. (Joel, Lines 14-18)

Joel's response explained his belief that God helped him deal with a negative life experience. Joseph described his knowledge of Voodoo and stated that he did not practice Voodoo. He expressed that he practiced Christianity and had a faith in God over Voodoo:

Voodoo is something I heard about back home in Haiti. Since I came to America, I don't hear much about Voodoo. I don't believe in Voodoo. I know there are good and bad spirits and people can use them against you or to hurt you or your family. I don't practice Voodoo, I practice Christianity. My God is bigger than Voodoo. I don’t practice Voodoo. (Joseph, Lines 20-24)

Like other participants, Joel described knowledge of Voodoo but since his arrival in the United States, he has adopted one of many mainstream religions, such as Christianity.

For example, Joseph explained why his spirituality is based upon his belief in God:

I believe in God because I was sick and God spared my life. God is the source of my life and health. I know God is going to make me better and I can go home from the hospital I can do nothing without him. When I was in Haiti, I was Catholic, but since I came to America, I gave my life to God and became a Christian. I love being a Christian. I am happy because I have God in my life. (Joseph, Lines 6-10)

All eight Haitian participants' responses described spirituality as a belief only in God. Haitians' beliefs focus on spirituality and divine predestination (Etienne \& PavlovichDanis, 2010). Their belief in God was described as a personal experience, a guide for dealing with life experiences and healing. Through the interpreter Rose described spirituality as a strong faith in God, "Spirituality means for me that ah when you place 
your faith in God and you're sure one hundred percent that he will do what He promise." (Rose, Line 3)

Lubin's spirituality as a resource helped him during a personal life experience.

Lubin's response described how his belief in God increased after he was hospitalized and in a coma, and how he was healed. As a result of that experience, Lubin was excited and likes to share his testimony of God's role in his life. Lubin's interpreter said:

"He doesn't care what religion you believe in but Lubin believes in God. He wants to preach to somebody. Lubin was in a coma for eight days. That's why I believe in God". (Lubin, Lines 63-65)

This response is an example of a strong belief in God and the role his spirituality played in his healing process. Like Lubin, other participants described their healing as a result of their belief in God. Kathy described spirituality as a belief in God and that she experienced his healing power. Kathy explained why she only believes in God. Kathy's interpreter explained, "Kathy believes only in God but why she does that is because when she's sick she call upon God and God answer her prayer" (Kathy, Line 10). Rose described her personal experience with God which was done through prayer. Rose expressed her belief that God will heal her. Rose interpreter explained:

"God, if you really can heal this person please give me a portion of what you are going to do for this person. Okay, give me please a portion of the benediction that you are, you are going to give to this person. I went to the prayer meeting and at the end okay something has changed. For awhile after this ah prayer meeting okay I ah, keep going to the church okay and pray a lot about my health. I never doubt about my healing even, ah, even though I without treatment, ok, I have ah, I was really confident that I will be healed by God."(Rose, Lines 18, 21)

Belief in God serves as a resource for coping and is common in the Haitian community. Kathy's response described a personal experience and the role of spirituality in her life, and how it helped her to cope with life experiences. Kathy's interpreter explained: 
"Spirituality is ah ah she considered to be a personal, very personal to her, is because sometimes she's cornered about obstacles in life about her condition and that instead of her feeling sad, feeling bad she had, she hears the voice that tells her to pray, pray. And then when she does pray, it releases a lot of her stress." (Kathy, Lines 15-19)

Kathy described how she was able to use her belief in God, incorporate the spiritual practice of prayer, and ease her anxiety as a result of her spiritual beliefs. Again, Rose discussed how her spiritual beliefs helped her to cope with life's challenges during her illness. Rose interpreter explained:

Spirituality help her to cope with not having a food to eat, not have a place to sleep and not having a lot of things but she thank God she take her medicine, she find people to help her, and she's able to come to the doctor. (Rose, Line 8)

The participants' responses to the role of spirituality in their lives all shared a belief in God who provides guidance, healing, and is a source for coping with illness and life experiences.

\section{Stigmatization of TB}

According to the Merriam-Webster dictionary (2010), stigma refers to a mark of shame or discredit. Lack of knowledge about TB may be a contributing factor to the stigmatization of TB. The stigma attached to TB is common in the Haitian community as well as it is in the African American community. In the Haitian community, fear of contagiousness may result in isolation of the patient imposed by the family (Mangan, 2009). Patients are required to stay in a separate house, not use eating utensils, and family members may not speak to the patient. This family-imposed isolation was evidenced by a male participant who became homeless after he told his family about his TB diagnosis. On the day of the interview with Patrick, he learned that he could not return to his cousin's home because of his cousin's concerns about his children being around the 
patient. Patrick became angry at the thought of his family abandonment during his illness. Patrick said "I don't have no family, don't ask me anymore questions" (Patrick, Line 34). Patrick decided that he did not want to be further interviewed and ended the interview and turned his back to me. Patrick became homeless and was discharged to a homeless shelter. The fear of abandonment that Patrick experienced may be a contributor to fear of disclosure to family. Joel explained, "I never really told anybody I have TB because I stay by myself. Nobody with me and only one person know that. That's my mom only. I told my mom I had TB" (Joel, Lines 37-38). TB is considered a bad disease to get, and people avoid being around TB patients due to the fear that they may contract the disease. Joseph explained:

TB is something bad to get. It makes you very sick and you can die from it. I heard of people with it, but I never though I would get it. People treat you different and do not want to be around you. (Joseph, Lines 30-33)

Joseph gave an example of the stigma attached to TB in the Haitian community. Lack of Knowledge

Lack of knowledge was common among the Haitian participants as well as the African Americans. Most of the Haitian participants mentioned cough as a symptom of TB, but could not verbalize other symptoms of the TB disease. A Haitian male believed a spell was placed on him and that caused him to develop TB. The notion of a spell as a cause for TB is a common rumor in the Haitian community. Joseph believed a spell was placed on him. However, Joseph contradicted himself when he explained, "I don't know. I think you breathe in the dust. I heard that people can put a spell on you and you get TB. I don't believe a spell was placed on me" (Joseph, Lines 34-35). Another participant 
verbalized the seriousness of TB if not properly treated. But he could not verbalize the causes for TB or the symptoms. Through the interpreter, Jean explained:

He doesn't know much about TB; however, he knows that TB will kill you. In Haiti, a lot of people have TB because they don't have enough care, they just die. But he knows that TB is real and that it kills. (Jean, Lines 26-30)

Again, lack of knowledge was evidenced by Kathy's response to the question of what causes TB and its signs and symptoms. Through the interpreter, Kathy said, "The dust from the sewing machines, life, and also she didn't eat well when she was in Haiti. So she figured those were the triggers to causing TB" (Kathy, Lines 31-32).

Malnutrition does place a person at risk for TB, but dust from a sewing machine is not a cause for TB as stated by Kathy. Again, most Haitian participants, just like African American participants, learned about TB from their healthcare provider after the diagnoses of TB were confirmed. Joseph stated that his healthcare providers and the health department staff provided information to him about TB. Joseph emphasized his belief that God would heal him. Joseph's response explained his learning experience and his faith in God:

I only learned about TB because I got TB. Before getting sick, I did not know or want to learn about TB. I learn about TB from the doctors and nurses here in the hospital told me. Also, the health department person told me about TB. Before coming to the hospital, I did not know much about TB. I read my Bible. I know God will heal me because I am a Christian. (Joseph, Lines 42-46)

Participants' responses to questions about TB show a lack of knowledge about TB signs and symptoms in the Haitian community. Because of this lack of knowledge, there are misconceptions about TB and stigma attached to TB. Participants' responses show that spirituality does not influence their knowledge of TB, but spirituality is used as a resource during their TB treatment. 


\section{Fear of Death}

Fear of death can either interfere or promote medication adherence for patients with long term illness (DiMatteo, 2004). Fear of death may lead to anxiety and worry for some patients. In addition to fear of death, belief in the effectiveness of TB medication contributed to Haitian participants' medication adherence. Seven of the Haitian participants verbalized a belief in the medications as motivation to be adherent with TB treatment. Fear of death and the will to live motivated all of the Haitian participants to be adherent with their TB medications. One participant, Joseph, said:

I know the medicine and prayer works because I feel better. I know if I don't take my medicines I will die. I have to live for my wife and children. I don't like feeling bad so I take my medicine so I don't feel sick, and I don't want to come back to the hospital. (Joseph, Lines 62-65)

Maggie and Marie both verbalized the same fear of death as motivation to be adherent with their TB medications. Through the interpreter, Maggie explained what medication adherence meant to her:

Medication adherence means, if she miss medications she might die. Once if you don't take it, every time you don't take it, you're losing one day of your life. Therefore, she'll take it everyday. (Maggie, Lines 64-66)

Through the interpreter, Marie explained her belief in the TB medication, "Everytime you don't take it, you're losing one day of your life. Therefore, she take it everyday" (Marie, Lines 92-93). However, one participant, Rose, shared a different belief about medication adherence. Rose explained:

To tell the truth, I'm sick and tired of taking medication and I don't take the medications. I ah, I keep my faith in God, and I know God will heal me no matter what. (Rose, Lines 77-80) 
Rose shared how her stronger belief in God did not motivate her to be adherent with her TB medications. Whereas, Jean and Errol expressed that their belief in the effectiveness of TB medications motivated them to be adherent with their TB medications. Jean said, "I believe in my medication. I know the minute that I stop taking the medication, I'm gonna feel bad" (Jean, Lines 58-60). Errolas well as Jean verbalized their belief in the effectiveness of TB medications. Errol said, "I take my TB medications in order to get well and live. I want to live until God calls me home" (Errol, Lines 33-34).

The Haitian participants' responses show that the will to feel better and live, fear of death, and belief in the effectiveness of the TB medications motivated them to be adherent with their medications. Despite having strong beliefs or faith in God, effectiveness of TB medication influenced the majority of participants to be adherent. Only one participant stated that she did not take her medication because her faith in God was bigger than faith in the medication effectiveness. The next section provides a discussion of similarities and differences across the two groups.

\section{Across Groups}

More similarities than differences in spirituality, stigma, knowledge, and medication adherence were found between African American and Haitian TB participants (see Table 4). 
The only difference found between the two groups was the Haitian participants' reference to knowledge of the practice of Voodoo and the belief that a spell had been placed on a Haitian male as the cause for TB.

Table 8

Description of Similarities and Differences across Groups

\begin{tabular}{ll}
\hline \multicolumn{1}{c}{ Similarities } & Differences \\
\hline Spirituality associated with a belief in God & $\begin{array}{c}\text { Acknowledgement of } \\
\text { Voodoo } \\
\text { Strong faith in God as a healer }\end{array}$ \\
Experienced stigmatization & Belief in spells \\
Lack of knowledge & \\
Desire to live and get better & \\
Fear of death & \\
Belief in effectiveness of TB medications
\end{tabular}

Summary

Thematic analysis was used to analyze the response to interview questions. Emerging from the data were four themes: God is in control, stigmatization of TB, lack of knowledge, and fear of death contributed to medication adherence. Interview responses confirmed the importance of spirituality in the lives of African Americans and Haitians. The themes that emerged from the interviews support the conceptual framework chosen for this study. Interestingly, during the analysis, the African American interview responses were more detailed than the Haitian's interview responses. African American 
participants appeared more at ease with an African American researcher. Despite the assistance of a Creole-speaking interpreter, the Haitian participants appeared less comfortable discussing their spirituality with an African American. The issue of an outsider gaining access to a minority community is discussed in the next chapter. Chapter 6 provides a discussion of the research questions, limitations, implications, and recommendations for future research. 


\section{CHAPTER VI}

\section{DISCUSSION}

This chapter is divided into three sections. The first section is a discussion of the research questions, including implications for practice and recommendations for further research. The second section is a discussion of the study's limitations. The final section is a summary.

\section{Discussion of the Research Questions}

This section provides a discussion of the findings of the analyses. Primary and secondary research questions are each presented and briefly discussed, followed by implications and recommendations for further research.

\section{Primary Research Question:}

What is the relationship between spirituality, knowledge of TB, and TB medication adherence among African Americans and Haitians?

Participants' responses on the Spiritual Perspective Scale (SPS) did not reveal a relationship between SPS and TB medication adherence. The relationship between spirituality and adherence among African Americans and Haitians ( $p=.208)$ was in the direction predicted: that is, greater medication adherence was associated with greater spirituality. It appears that spirituality may have a positive relationship with participants' decision making about medication adherence. As previously discussed, spirituality involves faith or willingness to believe, a search for purpose in life, a sense of connection with others or a transcendence of the self, resulting in a sense of inner peace and well being (Delgado, 2005; Mattis, 2005). This sense of inner peace and well being may have led to acceptance, which is a factor in medication adherence. Acceptance of their illness 
influences participants to seek medical care and take medications (Adams, Pill, \& Jones, 1997). However, I failed to reject the null in that there was not a statistically significant relationship between spirituality and TB medication adherence $(p=.208)$ among African Americans and Haitians. This may be the result of a small sample size; a larger sample size may reveal a statistically significant relationship.

African American participants' interviews did not reveal a relationship between spirituality, knowledge, and TB medication adherence. All 8 African American participants stated a belief in God or a higher being. Their belief in God served as a coping source that included beliefs and the ability to surrender to God and not worry about their illness. It appeared that spirituality served as a constant present resource in an individual's health (Reed, 1991) and helped participants cope with health concerns. African Americans' spirituality included spiritual practices, such as having faith and prayer. Participants verbalized that their spirituality served as a positive resource, helped them to interpret and make meaning out of life's experiences, and influenced changes in their behaviors. Despite participants' belief in God and spiritual practices, their beliefs and practices were not related to their knowledge of TB or medication adherence.

All 8 Haitian participants described spirituality as a belief in God. This belief in God is a personal experience and serves as a guide for dealing with life. Five of the Haitian participants described God as a healer. All participants verbalized that God served as a resource for coping with life and health issues. Haitian participants' interviews did not reveal a relationship between spirituality, knowledge and TB medication adherence. 


\section{Implications for Practice}

African American and Haitian participants verbalized the desire to be adherent with their TB medication and cure TB. Spirituality was a part of their daily lives but had no relationship with their TB medication adherence. Findings from this research can guide patients, healthcare professionals, and religious/spiritual leaders involved in the care of African American and Haitian TB patients. Better understanding of the patients' spirituality and its impact on health beliefs and practices helps to improve the patient healthcare provider relationship (Sulmasy, 2009), although spirituality can either interfere or have a positive relationship with a person's health. Results from this study support the literature about the positive relationship between spirituality and patients' health behavior practices. Educational guides can be developed to help healthcare professionals and religious leaders to eliminate some of the misconceptions about minority patients' beliefs and health practices. In addition, health education programs geared toward early identification and medication adherence for TB and other health conditions can be implemented through churches health ministries and community health fairs.

\section{Recommendations for Further Research}

Further research should examine the relationship between spirituality, knowledge, and TB medication adherence with a larger sample size. This will allow the researcher to get a better picture of the relationship between spirituality, knowledge, and TB medication adherence. Another study could include other ethnic groups such as Hispanics in the community to identify similarities and differences in spirituality and spiritual beliefs among TB patients. 
Research is needed on religious/spiritual leaders' and organizations' role in promoting health and medication adherence through the churches' healthcare ministry and community health fairs. Many researchers believe that certain beliefs, attitudes, and practices influence health and health behaviors. However, results from this study showed no relationship between spirituality, knowledge, and TB medication adherence. Increased interest among patients and providers in the role of spirituality and health may be due to dissatisfaction with the impersonal nature of our current healthcare system and the belief that medical science does not have the answer to every question about health and wellness (Newlin, Knafl, \& Melkus, 2002).

\section{Secondary Research Question 1:}

How do African Americans and Haitians perceive the role of spirituality in their lives after being diagnosed with TB?

The Spiritual Perspective Scale scores for African American and Haitian participants revealed a high spiritual perspective score among African Americans and Haitians. Participants' interview responses documented that spirituality was a key source of strength and motivation for African Americans and Haitians diagnosed with TB.

All African American participants' interview responses revealed how spirituality served as a coping strategy during their experience with TB as well as other stressful life events. Faith in God was a deeply held belief among African American participants. African American participants' responses showed a strong faith in God to heal them and help them during their experience with TB and other life experiences. In the African American community, spirituality helps individuals find meaning in illness, suffering, and death (Dyson, Cobb, \& Forman, 1997). Spirituality has been found to strongly 
influence the health beliefs of African Americans (Utsey et al., 2007). However, spirituality is not unique to African Americans.

All Haitian participants' responses revealed how spirituality served as a coping strategy during their experience with TB as well as other stressful life events. Haitian participants stated a strong faith in God as a healer. Their belief in God is a source of comfort that helped them cope with their experience with TB. Haitian participants stated that their faith and belief in God was stronger than Voodoo. Haitians in the United States are active in various mainstream religious groups, including Catholicism and Protestantism. However, many Christian Haitians denounce Voodoo (Desrosiers \& St. Fleurose, 2002; Etienne \& Pavlovich-Danis, 2010), which was evident in this study. Some of the Haitian participants verbalized the existence and knowledge of Voodoo; however, no participant stated that they practiced Voodoo. Overall findings of this study are consistent with the literature indicating that spirituality as well as religion has been an important mechanism by which African Americans and Haitians manage adverse life experiences.

Spirituality involves beliefs, behaviors, characteristics, and experiences that are grounded in and further developed by an individual's relationship with God or a transcendent force (Mattis, 2005). Both groups engaged in the spiritual practice of prayer. As found in previous studies, participants' experiences with TB led to becoming wiser, cautious, and concerned about their health (Hansel, Wu, Chang, \& Diette, 2004). Spirituality used as a resource during participants' experience with TB led to positive decision making about their health and medication adherence. Spiritual practices such as prayer, the act of putting oneself in the presence of or conversing with a higher power, 
has been used as a means of healing across all cultures (Newlin et al., 2002). Both African American and Haitian participants engaged in prayer during their experience with TB. Their spirituality served as a source of comfort, adding meaning to their lives and guidance during their experience with TB.

Implications for Practice

Healthcare professionals working with African American and Haitian patients must recognize spirituality as an important component of their lives and the role of spirituality in the process of recovery from a disease such as TB. A personalized approach to care is very important for both groups of participants. Healthcare professionals need to assess and pay attention to the role of spirituality in the lives of both African American and Haitian TB patients. Spiritual/religious leaders can be incorporated into healthcare professionals' educational interventions to help TB patients in the healing process.

Recommendations for Further Research

Now that there is growing belief and interest in the connection between spirituality and health, this is the opportune time for research in this field. Researchers should focus on the connection between spirituality and health, better understanding of why there is a connection, and how this connection works in various cultures. The outcome of research in this area will aid in the development of educational interventions for healthcare professionals, churches, spiritual leaders patients, and families dealing with TB and other diseases such as HIV/AIDS that are misunderstood and highly stigmatized. In addition, further research should include replication of this study with a different ethnic population affected with TB. 


\section{Secondary Research Question 2:}

How do African Americans and Haitians understand and learn about TB after diagnosis?

The majority of African American participants in this study received TB information after they were diagnosed with the disease. Four African American participants stated they had no knowledge about TB. Two African American participants stated they only became interested in learning about TB after they were diagnosed. In an effort to keep from being labeled as a TB patient, 3 African American participants stated they did not accept or read TB literature or pamphlets. TB has stigma attached to it since it is a disease that is not well understood among the public, symptoms cannot be concealed, and the patient is usually blamed for having TB. Stigma does not occur naturally and is usually created by individuals and communities due to their own fears (Goffman, 1963). In the African American community, TB is considered to be a dirty disease which attacks bad people (Jenkins, 1966). Interview responses from both African American and Haitian participants indicated that stigma is still attached to TB. TB patients were subjected to rejection by family as evidenced by several participants' responses in both groups. TB patients may be forced out of their homes or lose their jobs. Participants' responses revealed how TB continues to be a highly stigmatized disease in both groups.

Haitian participants also received TB information after they were diagnosed with the disease. All Haitians participants' interview responses revealed that they obtained inaccurate information from family and friends in their community. Lack of knowledge was common among the Haitian participants; for example, participants stated they developed TB as a result of no food, from breathing in dust from a sewing machine, and 
spells being placed on them. This lack of knowledge about TB may contribute to the stigma that exists in the Haitian community. Haitian participants verbalized fear of disclosing their illness due to the stigma attached to TB. Fear of disclosure of their illness due to the stigma attached has been shown to interfere with early identification and treatment (Coreil, 2001; Handel, Chang \& Diette, 2004; Heurtelou, 2001; Ilongo, 1994; Kelly, 1999). In addition, social stigma and discrimination by healthcare professionals have contributed to patients' lengthy delays in seeking treatment (Ilongo, 1994; Rubel \& Garro, 1992). This was evidenced by some of the participants in this study who did not seek care until they were acutely ill and required hospitalization. TB is a disease that can be treated in an outpatient setting. Fortunately, the stigma and misconceptions about TB did not lead to medication nonadherence among the study participants. Misconceptions about TB and Voodoo continue to exist in the Haitian community. Haitian participants in this study denied the existence of Voodoo; however, in the Haitian community, there is a belief that TB is the result of Voodoo spells being placed on a person (Etienne-PavlovichDanis, 2010; Farmer et al., 1991).

Spirituality has been viewed as a means by which adult learners acquire knowledge and create meaning (Dirkx, 1997, 2001). Knowledge acquisition was obtained by one-on-one instruction usually provided by the physician, TB nurse, or health department worker in the hospital or outpatient clinic. According the Health Belief Model (1966), when given the appropriate information, patients are capable of assessing susceptibility and efficacy and making a decision to comply with recommended treatment plans. This points to the need for healthcare professionals and patients to obtain accurate knowledge about TB infection, TB disease, signs/symptoms, transmission, and treatment. 
Obtaining accurate knowledge is an important aspect of TB control activities in the community. Inappropriate or insufficient knowledge regarding potential hazards of not engaging in a health behavior may result in nonadherence to treatment regimen (Leventhal \& Cameron, 1987).

Participants' responses showed how spirituality informed and guided them, and changed behaviors and emotions (Gallagher, 2006) during their experience with TB. It appears that for African Americans and Haitians, spirituality helped to improve their quality of life by influencing the way they cope with having a stigmatized disease such as TB.

\section{Implications for Practice}

African American and Haitian participants' responses indicated limited, or in some participants, no explicit knowledge as evidenced by the participants' lack of knowledge of TB signs, symptoms, and medications. Yang's (2003) Holistic theory of Knowledge and Learning helps adult educators understand the learning process in adult learners. The three knowledge facets — explicit, implicit, and emancipatory—were evident in each participant. Most of the African American and Haitian participants' responses indicated lack of knowledge about TB. This should be a concern for everyone, because lack of knowledge may contribute to a delay in seeking treatment, expensive hospitalization, and further transmission of TB in the community. Participants' implicit knowledge of TB medication effectiveness was shown through a health behavior, such as the high percentage (87\%) of medication adherence among both groups. Spirituality among both groups represented the emancipatory facet of knowledge. Interviews among 
participants revealed emotions, feelings, and spirituality for TB patients as an underlying factor in the lack of knowledge about TB.

The misconception about TB in the community and healthcare professionals related to participants' refusal to accept written material, such as handout or pamphlets. Participants verbalized feelings of shame and hid the diagnosis from family and friends to prevent being treated differently and labeled as a bad person or dirty person. Written health information (i.e., cough etiquette guide) that does not include specific disease names may help alleviate participants' refusal to accept written literature. In addition, positive behaviors and reinforcement from healthcare professionals may encourage learning about TB. Lack of knowledge about TB has been shown to be a challenge for patients and healthcare professionals. Healthcare professionals' fear of contracting TB may lead them to avoid interaction or appear impersonal with TB patients. Increased awareness through education should help ease healthcare professionals' fear of TB. In addition, cultural awareness of African American and Haitian religious or spiritual practices education is a must for healthcare professionals who provide care to various ethnic minority groups in healthcare settings.

Participants' responses about spirituality showed how it is an ever-present resource in African American and Haitian lives. Educators must recognize the spiritual aspect of participants' lives that can interfere or encourage learning. Spiritual beliefs must be recognized and when needed incorporated into the health education provided to TB patients. Healthcare staff education is needed to increase their knowledge and competence in order to provide quality care to TB patients and decrease their fear of patients with contagious diseases. Therefore, health education must be provided in a 
culturally sensitive manner, which can empower patients and healthcare staff with new information and, thus, influence their beliefs and decision making skills, and increase medication adherence.

Participants' interview responses highlight the stigma TB patients encountered once diagnosed with TB. Healthcare professionals must become aware of the role of bias and stigma attached to TB in the African American and Haitian community. Educational interventions must be geared toward healthcare professionals, patients, and community; it should be designed to eliminate misconceptions about TB, eliminate undue fear, and lessen the stigma attached to TB. To help reduce the stigma attached to $\mathrm{TB}$, mandatory training programs and regular refresher courses in TB control and management are required for healthcare professionals to disseminate correct information concerning $\mathrm{TB}$ transmission, diagnosis, isolation precautions, and treatment. In addition, the training programs should include an annual review of healthcare organizations' discrimination policies to convince healthcare professionals of the need to change old beliefs and habits. This TB information should be circulated widely throughout healthcare organizations, not just to those working with TB patients. Training and refresher programs may help to alleviate healthcare professionals' fear of the disease and provide a sense of security that may help improve the way they relate to TB patients. Former TB patients can serve as peer educators for healthcare professionals and patients to increase their awareness of this patient population and offer educational and psychosocial support to reduce myths, misconception and stigma attached to TB. 


\section{Recommendations for Further Research}

Written literature, such as hand outs and pamphlets, may not be an appropriate educational tool for patients diagnosed with TB. Further research is needed to identify appropriate educational interventions, such as use of peer educators in the community, healthcare organizations, and churches for minority populations, such as African American and Haitian TB patients. Lack of knowledge and stigma were found to be common factors among African American and Haitian TB patients. Both factors may have contributed to some participants' delay in seeking TB treatment. Further research is needed to determine whether increasing knowledge and reducing stigma in the African American and Haitian community both reduces delays in seeking treatment and improves patients' outcomes.

\section{Secondary Research Question 3:}

How do African Americans and Haitians understand the directions concerning TB medication adherence?

Eight African American participants verbalized acceptance and the need to do whatever was required to cure TB as reasons for taking their TB medications. Fear of death as a motivator to be adherent with TB medication was commonly stated among the African American participants According to Pound and colleagues (2005), patients take medications to obtain relief from symptoms, avoid hospitalizations, prevent disease progression, and be normal. Participants in this study verbalized the desire to obtain relief from symptoms, prevent disease progression, and avoid death. Therefore, communication between the healthcare professional and patients must include information about TB and TB medications. Acceptance is a major factor in adherence. Once the participants 
received the information about $\mathrm{TB}$ and $\mathrm{TB}$ medication, acceptance influenced their decision to take the medication. Acceptance of their illness and fear of death led African Americans participants in this study to be adherent with the TB medication regimen.

Haitians participants stated they learned from the physician and nurse that in order both to feel better and to live, they had to take TB medication. Seven of the Haitian participants verbalized a belief in the effectiveness of TB medication as motivation to be adherent with TB treatment. Again, fear of death was commonly stated among the Haitian participants.

Seventeen participants, 9 Haitians and 8 African Americans, refused to be interviewed due to reasons such as refusal to reveal personal information, lack of time, lack of memory, and one participant stated she did not want to relive that phase of her life. Nonadherence to TB medication was not found to be a problem with either group of participants in this study. Eighty-four percent of participants were adherent with their TB medications. African American and Haitian participants in this study verbalized their spiritual beliefs, which did not interfere with their belief in the effectiveness of the medication and their intent to adhere to the TB treatment. The theory of reasoned action (Ajzen \& Fishein, 1980) may explain the relationship between beliefs, intentions, and actions. This theory posits that behaviors such as mediation adherence are predicted by one's personal belief and intention to perform the behavior, medication adherence. Only one participant stated that belief in the effectiveness of the medications was motivation to be adherent with the treatment to cure TB and live. One participant stated that personal belief about spirituality was stronger than her belief in the effectiveness of TB medication; however, she did adhere with her TB treatment. 
McDonnell, Turner, and Weaver (2001) identified characteristics associated with TB medication, such as (a) satisfaction with interpersonal care, (b) good perception of the medication effectiveness, and (c) support from family and friends. Four out of the 33 participants were married and stated they receive support from their spouses. Most of the participants' responses indicated satisfaction with the interpersonal care they received during the course of their TB treatment and their belief in the effectiveness of TB medications. Participants' responses indicated that belief in the effectiveness of the TB medications contributed to their adherence to treatment. Two participants' responses indicated that they received support from family during their experience with TB. The stigma attached to TB may be a reason for limited or no support from family and friends. The desire to be cured from TB was a common goal for both African American and Haitian participants.

African Americans' and Haitians' belief in God's sovereignty over their health was evident by participants spiritualizing rather than medicalizing their health beliefs (King, Burgess, Akinyela, Counts-Spriggs, \& Parker, 2005). In this study, African American and Haitian participants used prayer to verbalize a belief and faith in a higher power such as God's will for them. Participants' responses indicated that the act of spiritualization of their health beliefs helped them cope with their experience with TB. Fortunately, the spiritualization of health beliefs did not interfere with the majority of participants' TB medication adherence. Participants did engage in the spiritual practice of prayer in addition to adhering with their TB medications. 


\section{Implications for Practice}

African American and Haitian participants' decisions to adhere with their TB medications can be explained by the Self-regulative System Theory (Leventhal \& Cameron, 1987). According to this theory, patients have the ability and are willing to self-regulate their behavior to obtain or maintain a healthy state. All participants in this study verbalized the desire to get well and live, which motivated them to be adherent with their TB medications. The participants understood their health status, that the goal of treatment was to cure $\mathrm{TB}$, and that this could be obtained by being adherent with $\mathrm{TB}$ medications. In this study, participants' spirituality did not interfere with communication between the patient and healthcare professional. Compliance or adherence behaviors depended on the clarity of communication between the patient and healthcare professionals during the education process. To enhance communication between the healthcare professional and participant, the researcher and the Creole-speaking Haitian interpreter was available in the clinic and hospital. Participants in the study received their education in either the outpatient clinic exam room or inpatient hospital room.

When addressing the problem of adherence to TB treatment, there is usually a one-way flow of information when this process should be one of negotiation and accommodation between provider and patient. Healthcare professional should encourage patients to discuss and listen to the patient's intent or plan of action to achieve medication adherence. Adherence is more likely if a patient understands the illness and has motivation and willingness to take part in a complex, long-term treatment regiment, which is elicited by the healthcare professional. When providing education to patients, healthcare professionals should address stigma-related issues and provide counseling 
before initiation of medications (Rintamaki, Davis, Skripkauskas, Bennett, \& Wolf, 2006). Educational interventions should be designed for healthcare professionals to improve the communication between patient and provider. In addition, when healthcare professionals show positive and encouraging attitudes and behaviors toward TB patients, this motivates them to adhere to the TB treatment (Dodor, Kelly, \& Neal, 2009). It is very important that healthcare professionals explore health beliefs with patients and allow them to express views about their health condition. When there is a language barrier, interpreters must be used to communicate with patients.

\section{Recommendations for Further Research}

A large scale study should be conducted at multiple locations in the community to explore the issue of spirituality, knowledge, and TB medication adherence among African Americans and Haitians. Further studies should be conducted to determine what patient, healthcare professional, and health care system factors mediate the relationship between lack of knowledge of TB, stigma, and TB medication adherence.

\section{Limitations}

There were two principal limitations to this study. The first limitation was the difficulties encountered recruiting participants to participate in the study. The study was designed to be conducted at two locations in the county - a large urban hospital and the health department clinic. Due to State of Florida Department of Health IRB rules, the researcher was not allowed to directly recruit health department patients for the study. The health department surveillance analyst had to initially identify potential participants and obtain consent from patients seen at the health department, which made recruitment of health department patients a challenge. Despite daily phone calls to remind the analyst, 
recruitment of health department patients was not aggressive or consistent. A major flood in the outpatient TB clinic led to the clinic being closed for one month, which then led to an unintended interruption in recruitment and data collection among participants. The downward decline in number of TB cases resulted in fewer TB patients being admitted to the hospital, and the shift in demographics of TB patients resulted in a small sample size. This limits the generalization of these results of the study to African American and Haitian TB patients in the community.

The second limitation of this study was Creole-speaking Haitian participants required the use of an interpreter. During the analysis of interview transcripts, I noticed that during some of the interviews the interpreter summarized the participants' responses instead - interpreting the participants' actual responses. Qualitative data analysis results revealed limited discussion of Voodoo among Haitian participants. Despite Voodoo being a recognized religion in Haiti, participants did not openly discuss this during the interviews. The response data provided may not reflect Haitian participants' actual beliefs and may not be a true reflection of their experience with Voodoo.

\section{Summary}

This mixed method study sought to explore the relationship between spirituality, knowledge, and TB medication adherence among African Americans and Haitians. To enhance the believability of the study findings, the quantitative phase addressed the what of the research question: What is the relationship between spirituality, knowledge of TB, and TB medication adherence among African Americans and Haitians? There was no relationship found between spirituality and TB medication adherence among African Americans and Haitians. Since no relationship was found in the quantitative phase of the 
study, the qualitative phase answered the how and why of the study through secondary research questions.

Secondary research questions examined the role of spirituality, knowledge of TB, and medication adherence among African Americans and Haitians. Four common themes emerged across both groups to answer the secondary research questions. Those themes were: (a) God is in control, (b) stigmatization of TB, (c) lack of knowledge, and (d) fear of death. The theme lack of knowledge about TB was found to contribute to stigmatization of TB patients. However, in this study stigma was not found to influence TB medication adherence.

Implications for practice, based on the findings, affect the curriculum and design of educational programs for healthcare professional, religious leaders and organizations, TB patients, and families. I learned that religious/spiritual leaders can become an important partner in the community to help inform their members about $\mathrm{TB}$ and other illnesses. Education is an important component in TB control activities in the community. Findings from this study provided empirical research evidence both to expand the cultural knowledge of healthcare educators and providers and community and religious leaders, and to allow them to discuss the effectiveness of understanding educational interventions provided to African Americans and Haitians diagnosed with TB. 


\section{REFERENCES}

Ablon, Joan. (1981). Stigmatized health conditions. Social Science \& Medicine, 158, 5-9.

Armstrong, T. D. (1996). Exploring spirituality: The development of the Armstrong3333 Measure of Spirituality. In R. L. Jones (Ed.), Handbook of tests and measurements for Black populations (pp. 105-115). Hampton, VA: Cobb and Henry.

Armstrong, T. D. (1998). The impact of spirituality on the coping process in families dealing with pediatric HIV or pediatric nephrotic syndrome (immune deficiency) (Doctoral dissertation, The University of North Carolina at Chapel Hill, 1998). Dissertation Abstracts International, 159, 64-82.

Armstrong, T. D., \& Crowther, M. R. (2002). Spirituality among older African Americans. Journal of Adult Development, 9, 3-12.

Bastable, S. B. (2003). Overview of education in health care. In S. B. Bastable (Ed.), Nurse as educator: Principles of teaching and learning for nursing practice $\left(^{\text {nd }}\right.$ ed., pp. 3-18). Boston: Jones and Bartlett.

Becker, M. H. (1976). Sociobehavioral determinants of compliance. In D. L. Sacket \& R. B. Haynes (Eds.), Compliance with therapeutic regimens (pp. 40-49). Baltimore: The John Hopkins University Press.

Becker, M. H., \& Maiman, L. A. (1975). Sociobehavioral determinants of compliance with health and medical care recommendations. Medical Care, 13, 10-24.

Bernard, H. R. (2000). Social research methods: Qualitative and quantitative approaches. Thousand Oaks, CA: Sage.

Bourguignon, E., \& Howard, M. (1998). The Haitians, the healers and the anthropologist: Two case studies. American Anthropologist, 100(3), 773-774.

Brislin, R. W. (1970). Back-translation for cross-cultural research. Journal of CrossCultural Psychology, 1, 185-216.

Britt, A.B. (2004, Summer). African Americans, substance abuse and spirituality. Feature Article. Retrieved May 14, 2010, from http://www.minoritynurse.com

Cameron, C. (1996). Patient compliance: Recognition of factors involved and suggestions for promoting compliance with therapeutic regimens. Journal of Advanced Nursing, 24, 244-250. 
Castro, A. \& Farmer, P. (2005). Understanding and addressing AIDS-related stigma: From anthropological theory to clinical practice in Haiti. American Journal of Public Health, 95 53-59.

Centers for Disease Control and Prevention. (1992). Prevention and control of tuberculosis with at-risk minority populations. Recommendations of the advisory council for the elimination of tuberculosis (Morbidity and Mortality Weekly Report 41/RR-5). Atlanta, GA: U.S. Department of Health and Human Services.

Center for Disease Control and Prevention. (1994). Improving patient adherence to tuberculosis treatment. Atlanta, GA: U.S. Department of Health and Human Services.

Centers for Disease Control and Prevention. (1998). Tuberculosis morbidity-United States, 1997. Atlanta, GA: U.S. Department of Health and Human Services.

Centers for Disease Control and Prevention. (2008). Reported tuberculosis in the United States, 2007. Atlanta, GA: U.S. Department of Health and Human Services.

Centers for Disease Control and Prevention. (2009). Reported tuberculosis in the United States, 2008. Atlanta, GA: U.S. Department of Health and Human Services.

Chachkes, E., \& Christ, G. (1996). Cross cultural issues in patient education. Patient Education \& Counseling, 27, 13-21.

Chapman, D. W., \& Carter, J. F. (1979). Translation procedures for the cross cultural use of measurement instruments. Educational Evaluation and Policy Analysis, 1(3), 71-76.

Chierici, R. C. (1991). Demele: “Making It” Migration and adaptation among Haitian boat people in the United States. New York: AMS.

Coarkes, S., \& Sellers, R. (2005). African American families as a context for racial socialization. In V. McLoyd, N. Hill, \& K. Dodge (Eds.), African American family life (pp. 265-280). New York: The Guilford Press.

Cohen, J. (1988). Statistical power analysis for the behavioral sciences. Hillside, NJ: Lawrence Erlbaum Associates.

Coreil, J. (2001). Haitian cultural study of TB/HIV Part 1. Tallahassee, FL: Florida Bureau of Tuberculosis and Refugee Health. 
Coreil, J., Lauzardo, M., \& Heurtelou, M. (2004). Cultural feasibility assessment of tuberculosis prevention among persons of Haitian origin in South Florida. Journal of Immigrant Health, 6(2), 63-69.

Creswell, J. W. (2003). Research design: Qualitative, quantitative and mixed methods Approaches (2nd ed.). Thousand Oaks, CA: Sage.

Cummings, K., Mohle-Boetani, J., Royce, S., \& Chin, D. (1998). Movement of tuberculosis patients and the failure to complete antituberculosis treatment. American Journal of Respiratory and Critical Care Medicine, 157(4), 1249-1252.

Dailey, D., \& Stewart, A. (2007). Psychometric characteristics of the spiritual perspective scale in pregnant African American women. Research in Nursing \& Health, 30(1), 61-71.

Delgado, C. (2005). A discussion of the concept of spirituality. Nursing Science Quarterly, 18(2), 157-162.

Desrosiers, A., \& Fleurose, S. (2002). Treating Haitian patients: Key cultural aspects. American Journal of Psychotherapy, 56(4), 508-521.

Diekhoff, G. M. (1996). Basic statistics for the social and behavioral science. Upper Saddle River, NJ: Prentice Hall.

DiMatteo, M. R. (1994). Enhancing patient adherence to medical recommendations. Journal of the American Medical Association, 271(1), 78-79,83.

DiMatteo, M. R. (2004). Variations in patients' adherence to medical recommendations: A quantitative review of 50 years of research. Medical Care, 42, 200-209.

DiMatteo, M. R., Hays, R. D., Gritz, E. R., Bastani, R., Crane, L., Elashoff, R., et al. (1993). Patient adherence to cancer control regimens: Scale development and initial validation. Psychological Assessment, 5, 102-112.

Dirkx, J. M. (1997). Nurturing soul in adult learning. In S. Imel \& R. G. Brockett (Series Eds.) \& P. M. Cranton (Vol. Ed.), New directions for adult and continuing Education: No. 74. Transformative learning in action (pp. 79-88). San Francisco: Jossey-Bass.

Dirkx, J. M. (2001). The power of feelings: Emotions, imagination, and the construction of meaning in adult learning. In S. Imel (Series Ed.) \& S. B. Merriam (Vol. Ed.), New directions for adult and continuing education: No. 89. The new update on adult learning theory (pp. 63-72). San Francisco: Jossey-Bass. 
Dodor, E.A., Kelly, S. \& Neal, K. (2009). Health professionals as stigmatisers of tuberculosis: Insights from community members and patients with TB in an urban district in Ghana. Psychology, Health \& Medicine, 14(3), 301-310.

Dyson, J., Cobb, M., \& Forman, D. (1997). The meaning of spirituality: A literature review. Journal of Advanced Nursing, 26, 1183-1188.

Edginton, M. E., Sekatane, C. S., \& Goldstein, S. J. (2002). Patients' beliefs: Do they affect tuberculosis control? A study in a rural district of South Africa. International Journal of Tuberculosis Lung Disease, 6(12), 1075-1082.

Edwards, L. (2006). Perceived social support and HIV/AIDS medication adherence among African American women. Qualitative Health Research, 16, 679-691.

Ellis, J., Beyers, N., Bester, D., Gie, R., \& Donald, P. (1997). Sociological and anthropological factors related to the community management of tuberculosis in the Western Cape communities of Ravensmead and Uitsig. South African Medical Journal, 87, 1047-1051.

English, L., Fenwick, T., \& Parsons, J. (2003). Spirituality of adult education and training. Malabar, FL: Krieger.

English, L., \& Gillen, M. (2000). A postmodern approach to adult religious education. In A. Wilson \& E. Hayes (Eds.), Handbook of adult and continuing education (pp. 523-538). San Francisco: Jossey-Bass.

Etienne, M. \& Pavlovich-Danis, S. (2010). Cultural considerations for Haitian patients. Nursing Spectrum: Florida, 20(4), 26-31.

Farmer, P. E. (2000). The consumption of the poor: Tuberculosis in the $21^{\text {st }}$ century. Ethnography, 1(2), 183-216.

Farmer, P., Simon, R., Ramilus, S., \& Kim, J. (1991). Tuberculosis, poverty, and "compliance": Lesson from rural Haiti. Seminars in Respiratory Infections, 6(4), 254-260.

Florida Department of Health, Bureau of Tuberculosis and Refugee Health. (n.d.). 2006 tuberculosis fact sheet, Miami-Dade County. Retrieved April 14, 2008, from http://www.doh.state.fl.us/disease control/tb/

Florida Department of Health, Bureau of Tuberculosis and Refugee Health. (n.d.). 2007 tuberculosis fact sheet, Miami-Dade County. Retrieved April 14, 2008, from http://www.doh.state.fl.us/disease control/tb/ 
Gallagher, S. (2006). Theological reflection at work: A phenomenological study of learning processes (Doctoral dissertation, Florida International University, 2006). Proquest Dissertations \& Theses. (AAT 3217568).

Gallagher, S., Rocco, T., \& Landorf, H. (2007). A phenomenological study of spirituality and learning processes at work: Exploring the holistic theory of knowledge and learning. Human Resource Development Quarterly, 18(4), 457480.

Gall, T., Charbonneau, C., Clarke, N., Grant, K., Joseph, A., \& Shouldice, L. (2005). Understanding the nature and role of spirituality in relation to coping and health. Canadian Psychology, 46(2), 88-104.

Gao, X., Nau, D. P., Rosenbluth, S. A., Scott, V., \& Woodward, C. (2000). The relationship of disease severity, health beliefs and medication adherence among HIV patients. AIDS Care, 12(4), 387-398.

Ginsberg, A. M. (1998). TB update: The tuberculosis scientific challenges and opportunities. Public Health Reports, 113, 128-136.

Goffman, E. (1963). Stigma: Notes on the Management of Spoilt Identity. London. Penguin.

Gray, J. (1997). Spiritual perspective \& social support in women with HIV infection: Pilot study. Journal of Nursing Scholarship, 29(1), 97.

Green, B., Lewis, R., Wang, M., Person, S., \& Rivers, B. (2004). Powerlessness, destiny, and control: The influence of health behaviors of African Americans. Journal of Community Health, 29(1), 15-27.

Greene, J. C., Caracelli, V. J., \& Graham, W. F. (1989). Toward a conceptual framework for mixed method evaluation designs. Educational Evaluation and Policy Analysis, 11, 255-274.

Hakala, K. (2007). Innovative teaching strategies in nursing and related health professions. In M. J. Bradshaw \& A. J. Lowenstein (Eds.) Teaching valuesensitive subjects ( $4^{\text {th }}$ ed., pp. 93-104). Boston, MA: Jones and Bartlet.

Halverson, P. K., Mays, G. P., Miller, C. A., Kaluzny, A. D., \& Richards, T. B. (1997). Managed care and the public health challenge of tuberculosis. Public Health Reports, 112, 22-28.

Hansel, N. N., Wu, A., Chang, B., \& Diette, G. (2004). Quality of life in tuberculosis: Patient and provider perspectives. Quality of Life Research, 13, 639-652. 
Heurtelou, M. (2001). Haitian cultural study of TB/HIV Part 2. Tallahassee, FL: Florida Bureau of Tuberculosis and Refugee Health.

Ilongo, I. (1994). Knowledge and beliefs of patients who are suspects or smear- and culture-positive for tuberculosis in Manhattan's central Harlem district in New York City (Doctoral Dissertation, Columbia University, 1994). Dissertation Abstracts International, 96, 638.

Janz, N. K., \& Becker, M. H. (1984). The health belief model: A decade later. Health Education Quarterly, 11, 1-47.

Jenkins, C. D. (1966). Group differences in perception: A study of community beliefs and feelings about tuberculosis. The American Journal of Sociology, 71(4), 417-429.

Johnson, K., Elbert-Avila, K., \& Tulsky, J. (2005). The influence of spiritual beliefs and practices on the treatment preferences of African-Americans: A review of the literature. Journal of the American Geriatrics Society, 53, 711-719.

Johnson, M. J., Williams, M., \& Marshall, E. S. (1999). Adherent and nonadherent medication-taking in elderly hypertensive patients. Clinical Nursing Research, $8(4), 318-335$.

Jones, E. (1987). Translation of quantitative measures for use in cross-cultural research. Nursing Research, 36(5), 324-326.

Kapborg, I., \& Bertero, C. (2002). Using an interpreter in qualitative interview: Does it threaten validity? Nursing Inquiry, 9(1), 52-56.

Kelly, P. (1999). Isolation and stigma: The experience of patients with active tuberculosis. Journal of Community Health Nursing, 16(4), 233-241.

King, D., \& Bushwick, B. (1994). Beliefs and attitudes of hospital inpatients about faith healing and prayer. Journal of Family Practice, 39(4), 349-352.

King, S., Burgess, E., Akinyela, M., Counts-Spriggs., \& Parker, N. (2005). Your body is God's temple. Research on Aging, 27(4), 420-446.

Kirschner, M. (2003). Spirituality and health. American Journal of Public Health 93(2), 185-186.

Kirscht, J. P. (1974). The health belief model and illness behavior. In M. H. Becker (Ed.), The health belief model and personal health behavior (pp. 60-82). Thorofare, NJ: Charles B. Slack. 
Kitazawa, S. (1995). Tuberculosis health education needs in homeless shelters. Public Health Nursing, 12, 409-416.

Koenig, H. (2001). Spiritual assessment in medical practice. American Family Physician, 63(1), 81.

Koenig, H., George, L., Titus, P., \& Meador, K. (2003). Religion, spirituality, and health service use by older hospitalized patients. Journal of Religion and Health, 42(4), 301-314.

Koenig, H., McCullough, M., \& Larson, D. (2000). Handbook of religion and health. New York: Oxford University Press.

Laws, M. B., Wilson, I. B., Bowser, D. M., \& Kerr, S. E. (2000). Taking antiretroviral therapy for HIV infection. Journal of General Internal Medicine, 15, 848-858.

Leventhal, H. (1993). Theories of compliance, and turning necessities into preferences: Application to adolescent health action. In N. A. Krasnegor, L. Epstein, S. B. Johnson, \& S. J. Yaffe (Eds.), Developmental aspects of health compliance behavior (pp. 91-121). Hillside NJ: Lawrence Erlbaum Associates.

Levin, J., \& Fox, J. (2003). Elementary statistics in social research $\left(9^{\text {th }}\right.$ ed.). Boston: Allyn and Bacon.

Lewis, R., \& Green, B. (2000). Assessing the health attitudes, beliefs, and behaviors of African-Americans attending church: A comparison from two communities. Journal of Community Health, 25(3), 211-224.

Miller, K.E. (2004). Beyond the frontstage: Trust, access, and the relational context in Research with refugee communities. American Journal of Community Psychology, 33(3-4), 217-227.

Maiman, L. A., \& Becker, M. H. (1974). The health belief model: Origins and correlates. In M. H. Becker (Ed.), The health belief model and personal health behavior (pp. 21-25). Thorofare, NJ: Charles B. Slack.

Maneesriwongul, W., \& Dixon, J. K. (2004). Instruments translation process: A methods review. Journal of Advanced Nursing, 48(2), 175-186.

Mangan, J. (2009). Haiti: Cultural competency and tuberculosis control. Gainesville, FL: University of Florida, The Southeastern National Tuberculosis Center at the University of Florida. 
Marks, L., Nesteruk, O., Swanson, M., Garrison, B., \& Davis, T. (2005). Religion and health among African Americans: A qualitative examination. Research on Aging, 27(4), 447-474.

Mattis, J. (2005). Religion in African American life. In V. C. McLoyd, N. E. Hill, \& K. A. Dodge (Eds.), African American family life: Ecological and cultural diversity (pp. 189-190). New York: The Guilford Press.

McDonnell, M., Turner, J., \& Weaver, M. T. (2001). Antecedents to adherence to antituberculosis therapy. Public Health Nursing, 18(6), 392-400.

Meraviglia, M. (2006). Effects of spirituality in breast cancer survivors. Oncology Nursing Forum, 33(1), E1-7.

Merriam, S. B., \& Simpson, E. L. (2000). A guide to research for educators and trainers of adults $\left(2^{\text {nd }}\right.$ ed.). Malabar, FL: Krieger.

Merriam-Webster's Online Dictionary?????? ()Stigma Retrieved on 5/15/10

Metcalf, C. A., Bradshaw, D., \& Stindt, W. W. (1990). Knowledge and beliefs about tuberculosis among non-working women in Ravensmead, Cape Town. South African Medical Journal, 77, 408- 411.

Miami-Dade County Tuberculosis Data. (2008). Miami, FL: Miami-Dade County Department of Health TB Unit. Retrieved March 20, 2010 from http://www.dadehealth.org/tb/TBdata.asp

Miller, M. A. (1995). Culture, spirituality, and women's health. Journal of Obstetric, Gynecology and Neonatal Nursing, 24(3), 257-263.

Miller, W. R. (Ed.). (1999). Integrating spirituality into treatment. Washington, DC: American Psychological Association.

Miller, W. R., \& Thoresen, C. E. (2003). Spirituality, religion and health. American Psychologist, 58(1), 24-35.

Morse, J. M. (2000). Determining sample size. Qualitative Health Research, 10(1), 3-5.

Moustakas, C. (1994). Phenomenological research methods. Thousand Oaks, CA: Sage.

Munhall, P. L. (2007). Nursing research: A qualitative perspective ( $4^{\text {th }}$ ed.). Sudbury, MA: Jones and Bartlett. 
Musgrave, C., Easley Allen, C., \& Allen, G. (2002). Spirituality and health for women of color. American Journal of Public Health, 92(4), 557-560.

Newlin, K., KNafl, K., \& Melkus, G. (2002). African-American spirituality: a concept analysis. Advances in Nursing Science, 25(2), 57-70.

Newman, I., \& Benz, C. (1998). Qualitative-quantitative research methodology: Exploring the interactive continuum. Carbondale and Edwardsville, IL: Southern Illinois University Press.

Nieswiadomy, R. M. (2008). Foundations of nursing research (5 $5^{\text {th }}$ ed.). Upper Saddle River, NJ: Pearson Education.

Nuwaha, F. (1997). Factors influencing completion of treatment among tuberculosis patients in Mbarara District, Uganda. East African Medical Journal, 74(11), 690693.

Ogbu, J., \& Simons, H. (1998). Voluntary and involuntary minorities: A cultural ecological theory of school performance with some implications for education. Anthropology \& Education Quarterly, 29(2), 155-188.

Oser, M. L. (2006). Patient education to promote adherence. In W. M. O’Donohue \& E. R. Levensky (Eds.), Promoting treatment adherence: A practical handbook for health (pp. 85-88). Sherman Oaks, CA: Sage.

Pamphile, L. (2001). Haitians and African Americans: A heritage of tragedy and hope. Gainesville, FL: University Press of Florida.

Patton, M. Q. (2002). Qualitative research and evaluation methods ( $3^{\text {rd }}$ ed.). Thousand Oaks, CA: Sage.

Pinkney, A. (2000). Black Americans (5th ed.). Upper Saddle River, NJ: Prentice-Hall.

Pitchforth, E., \& van Teijlingen, E. (2005). International public health research involving interpreters: A case study from Bangladesh. Biomed Central Public Health, 5(71), 698-705.

Polit, D. F., \& Beck, C. T. (2006). Essentials of nursing research (6 ${ }^{\text {th }}$ ed.). Philadelphia, PA: Lippincott.

Polit, D. F., Beck, C. T., \& Hungler, B. P. (2001). Analyzing quantitative data. In D. F. Polit \& C. T. Beck (Eds.), Essentials of nursing research (5 ${ }^{\text {th }}$ ed., pp. 327-370). Philadelphia, PA: Lippincott. 
Pound, P., Britten, N., Morgan, M., Yardley, L., Pope, C., Daker-White, G. \& Campbell, R. (2005). Resisting medication: A synthesis of qualitative studies of medicine taking. Social Science \& Medicine, 61,133-155.

Poutin, D. (2000). Interview. In D. Cormack (Ed.), The research process in nursing (4 ${ }^{\text {th }}$ ed., pp. 292-293). Malden, MA: Blackwell Science.

Reed, P. G. (1992). An emerging paradigm for the investigation of spirituality in nursing. Research in Nursing and Health, 15, 349-357.

Reed, P. G. (1991). Preferences for spiritually related nursing interventions. Applied Nursing Research, 4(3), 122-128.

Reed, P. G. (1986). Religiousness among terminally ill and health adults. Research in Nursing and Health, 9, 35-42.

Richards, E. (2003). Motivation, compliance and health behaviors of the learner. In S. B. Bastable (Ed.), Nurse as educator: principles of teaching and learning ( $2^{\text {nd }}$ ed., pp. 162-183). Boston: Jones and Bartlett Publishers.

Rintamaki, L., Davis, T., Skripkauskas, S., Bennett, C. \& Wolf. M. (2006). Social stigma concerns and HIV medication adherence. AIDS Patient Care and STDs, 20, (5), 359-368.

Rosenstock, I. M. (1974). Historical origins of the health belief model. In M. H. Becker (Ed.), The health belief model and personal health behavior (pp. 1-8). Thorofare, NJ: Charles B. Slack.

Rubel, A., \& Garro, L. (1992). Social and cultural factors in the successful control of tuberculosis. Public Health Reports, 107(6), 626-635.

Rudd, P. (1993). The measurement of compliance: Medication taking. In N. A.

Krasnegor, L. Epstein, S. B.Johnson \& S. J.Yaffe (Eds.), Developmental aspects of health compliance behavior (pp. 185-209). Hillside NJ: Lawrence Erlbaum Associates.

Sackett, D. L. (1976). The magnitude of compliance and noncompliance. In D. L Sackett, \& R. B. Haynes (Eds.), Compliance with therapeutic regimens (pp. 9-25). Baltimore, MD: The John Hopkins University Press.

San Sebastian, M., \& Bothamley, G. H. (2000). Tuberculosis preventive therapy: Perspective from a multi-ethnic community. Respiratory Medicine, 94, 648-653. 
Steen, T. W., \& Mazonde, G. N. (1999). Ngaka ye setswana, ngaka ya sekgoa or both? Health seeking behaviour in Batswana with pulmonary tuberculosis. Social Science \& Medicine, 48, 163-172.

Sulmasy, D.P. (2009). Spirituality, religion, and clinical care. Chest, 135(6), 1634-1642.

Sumartojo, E. (1993). When tuberculosis treatment fails: A social behavioral account of patient adherence. Annual Review of Respiratory Disease, 147, 1311-1320.

Tashakkori, A., \& Teddlie, C. (1998). Mixed methodology: Combining qualitative and quantitative approaches. In L. Bickman \& D. Rog (Eds.), Applied social research method series (pp. 43-58). Thousand Oaks, CA: Sage.

Teddlie, C., \& Tashakkori, A. (2009). Foundations of mixed methods research: Integrating quantitative and qualitative approaches in the social and behavioral sciences. Thousand Oaks, CA: Sage.

Thoresen, C., \& Harris, A. (2002). Spirituality and health: What's the evidence and what's needed? Annals of Behavioral Medicine, 24(1), 3-13.

Tisdell, E. J. (2001). Spirituality in adult and higher education. Columbus, OH: ERIC Clearinghouse on Adult Career and Vocational Education. (ERIC Document Reproduction Service No. ED459370)

Tisdell, E. J. (2003). Exploring spirituality and culture in adult and higher education. San Francisco: Jossey-Bass.

Trotter, J. W. (2001). The African American experience. Boston: Houghton Mifflin.

Usher, K. (2001). Taking neuroleptic medications as the treatment for schizophrenia: A phenomenological study. Australian and New Zealand Journal of Mental Health Nursing, 10, 145-155.

Utsey, S., Bolden, M., Willimas, O., Lee, A. Lanier, Y., \& Newsome, C. (2007). Spiritual Well-being as a mediator of the relation between culture-specific coping and Quality of life in a community sample of African Americans. Journal of CrossCultural Psychology, 38(2), 123-136.

Vermeire, E., Hearnshaw, H., \& Van Royen, P. (2001). Patient adherence to treatment: Three decades of research. A comprehensive review. Journal of Clinical Pharmacy and Therapeutics, 26, 331-342.

Walsh, S., Hagan, T., \& Gamsu, D. (2000). Rescuer and rescued: Applying a cognitive analytic perspective to explore the mis-management of asthma. British Journal of Medical Psychology, 73, 151-168. 
Weidman, Hazel. (1978). Miami Health Ecology Project: A Statement on Ehtnicity and Health,1. Miami, FL. University of Miami School of Medicine.

Wesley, M.G. (1997). Community primary prevention intervention of chemical dependency for African American families and communities: A collaborative nursingfacilitation. Journal of Addictions Nursing, 9 (3), 129-135.

Westaway, M. S. (1990). Knowledge and attitudes about tuberculosis of black hospitalized TB patients. Tubercle, 71, 55-59.

Westlake, C., Dracup, K, Creaser, J., Livingston, N., Heywood, T., Huiskers, B., Fonarow, G. \& Hamilton, M. (2002). Correlates of health-related quality of life in patients with heart failure. Heart \& Lung, 31(2), 85-93.

Wolfe, H., Marmor, M., Maslansky, R., Nichols, S. Simberkoff, M., Des Jarlais, D. et al. (1995). Tuberculosis knowledge among New York City injection drug users. American Journal of Public Health, 85(7), 985-988.

Yang, B. (2003). Toward a holistic theory of knowledge and adult learning. Human Resource Development Review, 2(2), 106-129.

Zuzelo, P. P. (2007). Evidence-based nursing and qualitative research. A partnership imperative for real-world practice. In P. L. Munhall (Ed.), Nursing research: $A$ qualitative perspective ( $4^{\text {th }}$ ed., pp 481-497). Sudbury, MA: Jones and Bartlett. 


\section{Appendix A}

\section{Code No.}

\section{SPIRITUAL PERSPECTIVE SCALE CReed, 1986}

Introduction and Directions: In general, spirituality refers to an awareness of one's inner self and a sense of connection to a higher being, nature, others, or to some purpose greater than oneself. I am interested in your responses to the questions below about spirituality as it may relate to your life. There are no right or wrong answers. Answer each question to the best of your ability by marking an " $X$ " in the space above that group of words that best describes you.

1. In talking with your family or friends, how often do you mention spiritual matters?

$\overline{\text { Not at all }} \frac{/}{\text { Less than once a year }} \frac{/}{\text { About once a year }} \frac{/}{\text { About once a month }} \frac{/}{\text { About once a week }} \frac{}{\text { About once a day }}$

2. How often do you share with others the problems and joys of living according to your spiritual beliefs?

$\overline{\text { Not at all }} \frac{/}{\text { Less than once a year }} \frac{/}{\text { About once a year }} \frac{/}{\text { About once a month }} \frac{/}{\text { About once a week }} \frac{}{\text { About once a day }}$

3. How often do you read spiritually-related material?

$\overline{\text { Not at all }} \frac{/}{\text { Less than once a year }} \frac{/}{\text { About once a year }} \frac{/}{\text { About once a month }} \frac{/}{\text { About once a week }} \frac{}{\text { About once a day }}$

4. How often do you engage in private prayer or meditation?

$\overline{\text { Not at all }} \frac{/}{\text { Less than once a year }} \frac{/}{\text { About once a year }} \frac{/}{\text { About once a month }} \frac{/}{\text { About once a week }} \frac{}{\text { About once a day }}$

5. Forgiveness is an important part of my spirituality.

$\overline{\text { Strongly Disagree }} \stackrel{\text { Disagree }}{\text { Disagree more than agree }} \frac{/}{\text { Agree more than disagree }}$ Agree Strongly $\frac{1}{\text { Agree }}$

6. I seek spiritual guidance in making decisions in my everyday life.

$\overline{\text { Strongly Disagree }} \frac{/}{\text { Disagree }} \frac{/}{\text { Disagree more than agree }} \frac{/}{\text { Agree more than disagree }}$ Agree Strongly $\frac{1}{\text { Agree }}$


7. My spirituality is a significant part of my life.

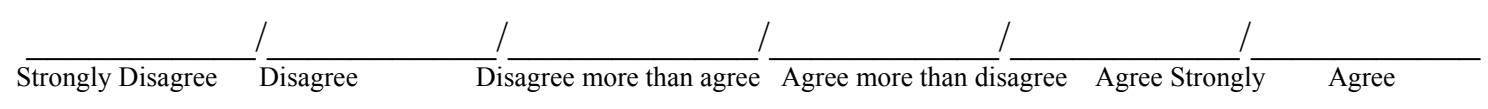

8. I frequently feel very close to God or a "higher power" in prayer, during public worship, or at important moments in my daily life.

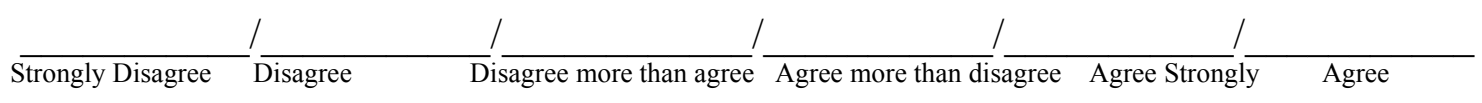

9. My spiritual views have had an influence upon my life.

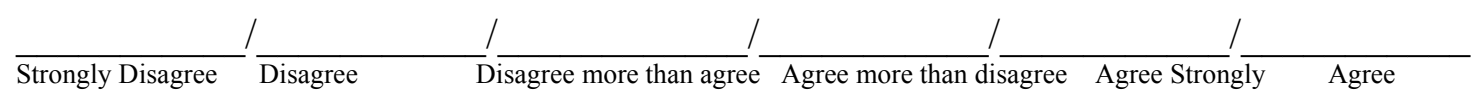

10. My spirituality is especially important to me because it answers many questions about the meaning of life.

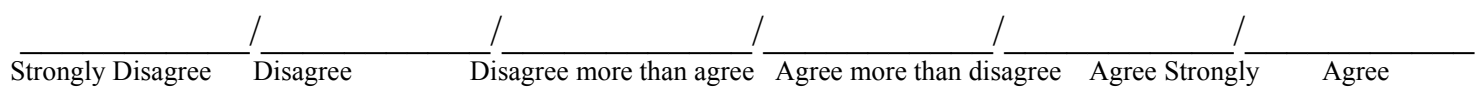

If possible, please describe how you define spirituality on the back of this page, or provide any other comments you feel are important for the researcher to know about.

Thank you for completing the SPS.

CReed, 1986 
Appendix B

Medication Administration Record

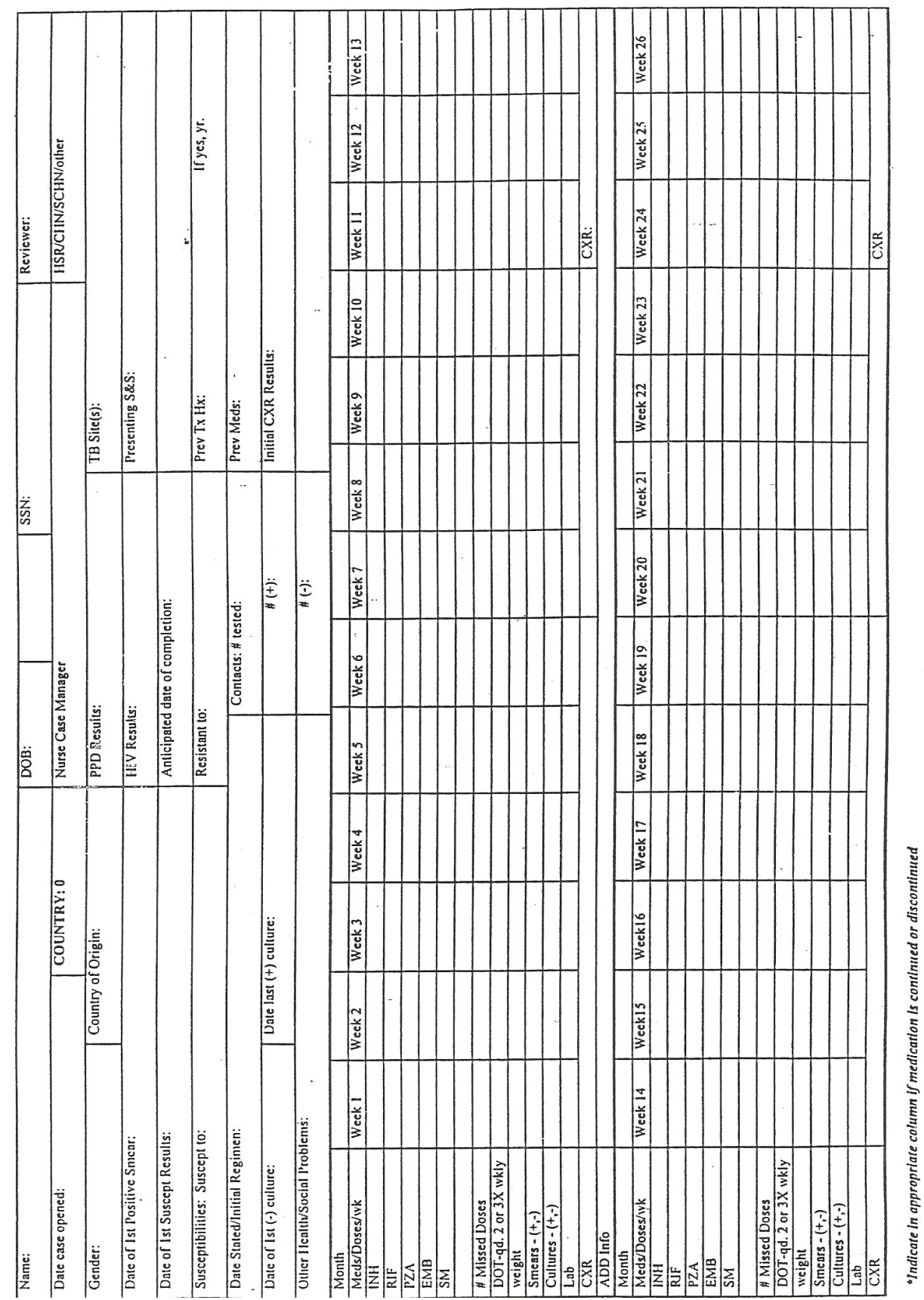




\section{Appendix C}

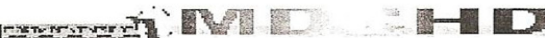
HEALTH WHWI-DADE COUNTYHEALTHDEPT. TB CASE/SUSPECT REPORT

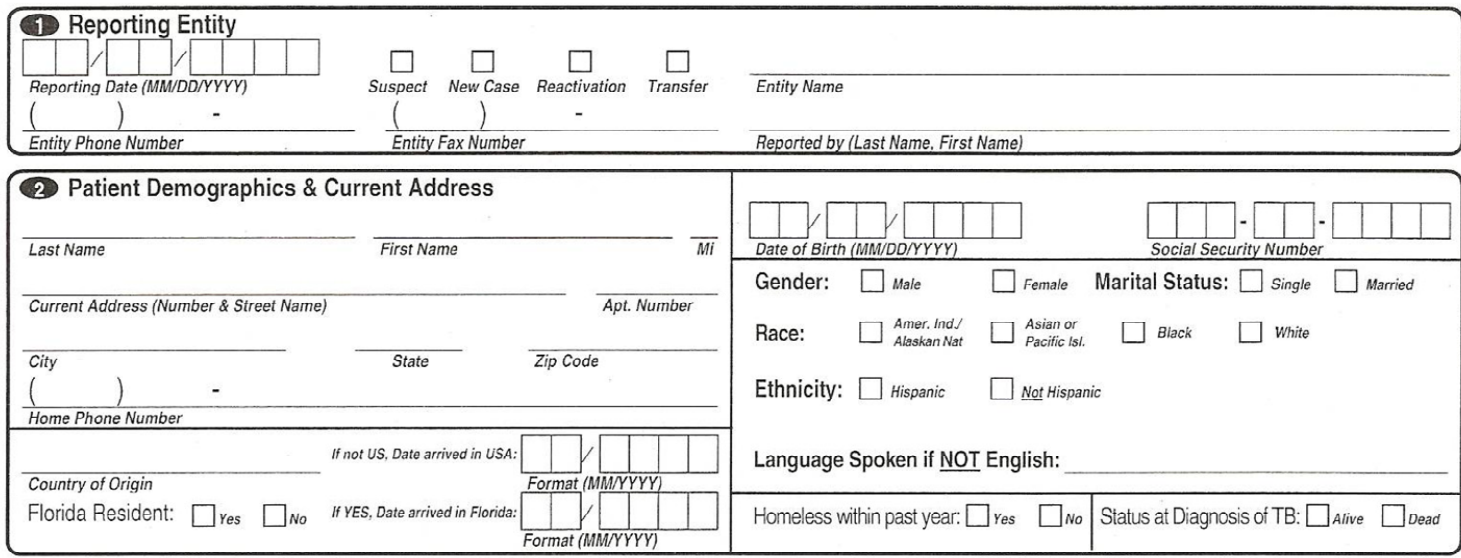

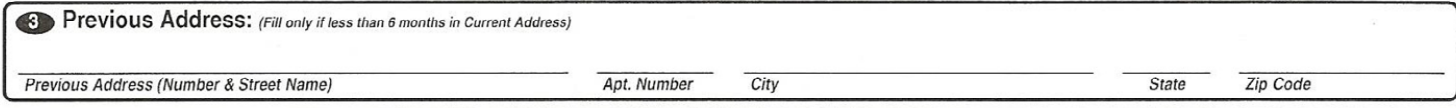

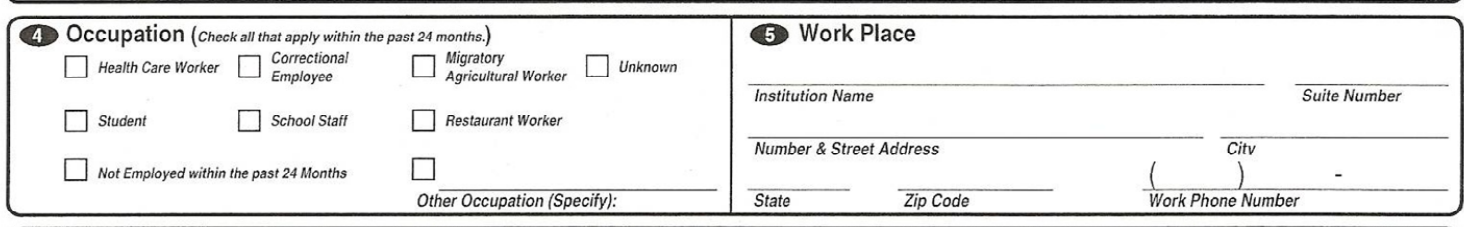

\begin{tabular}{|c|c|c|c|}
\hline \multicolumn{3}{|c|}{ (6) Past Medical (TB) History } & \\
\hline$\square$ Yes $\quad \square$ No & & & \\
\hline Previous Treatment & If Yes, When (year) & & County, State or Country \\
\hline Duration of $R x$ & aken: $\square_{\text {inrug }}$ & $\square_{2 \text { or more }}$ & Specify (drug name) \\
\hline
\end{tabular}

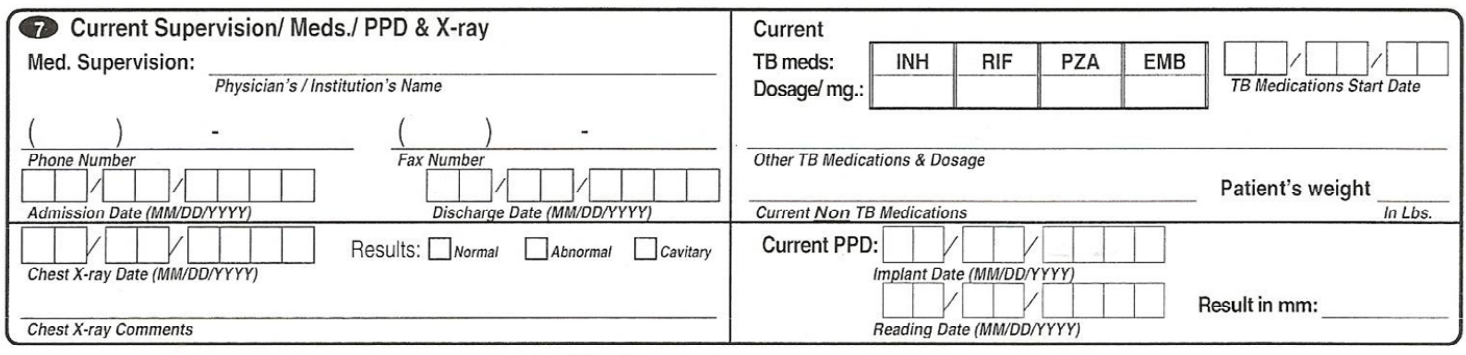

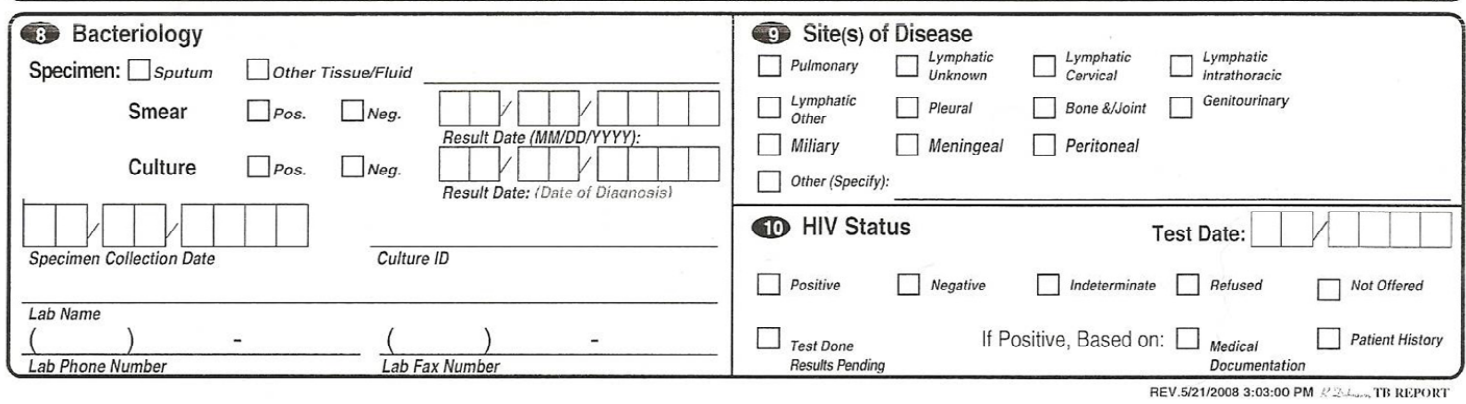




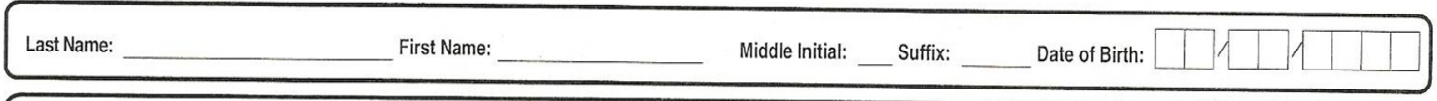

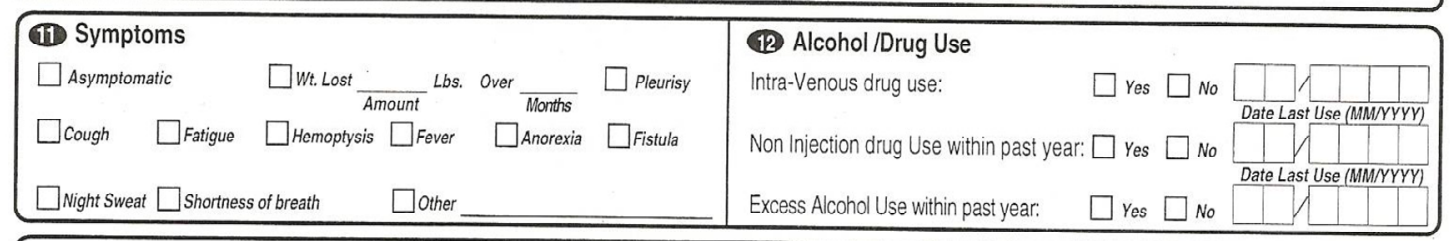

13 Contact to TB Case
Ever Exposed to a TB Case? $\square$ Yes $\square$ No How Long?
Did any family member die with TB? $\square$ Yes $\square$ No

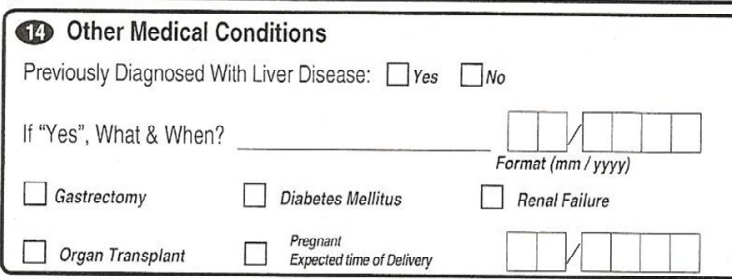

$\begin{array}{ll}\square \text { Epilepsy } & \square \text { Allergies } \\ \square \text { Immunosuppressive Medications } & \square \text { Silicosis (Occupational Lung Disease) } \\ \square \text { Jejuoileal Bypass } & \square \text { Cancer of Head, Neck or Lung } \\ \text { Other, Specify } & \end{array}$

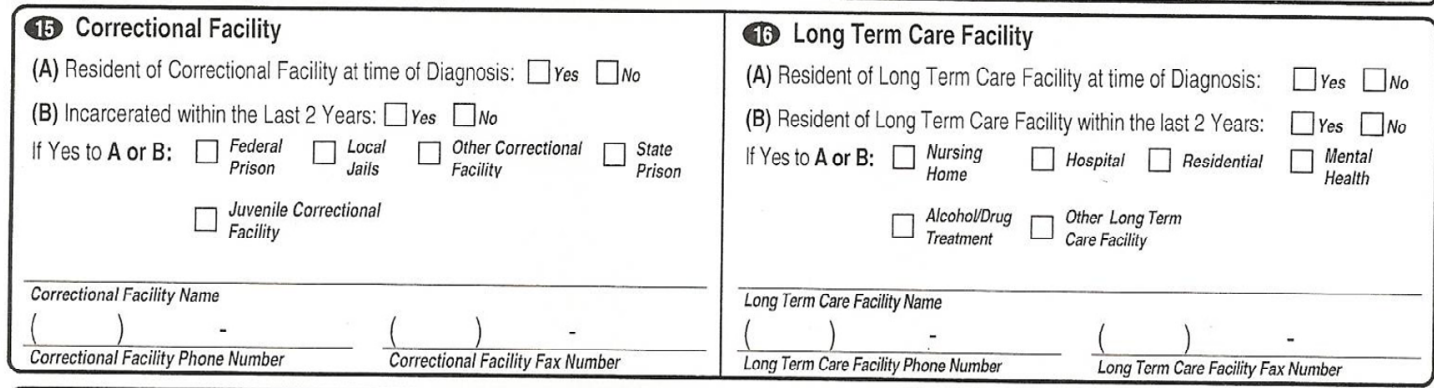

\begin{tabular}{|c|c|c|c|c|c|}
\hline \multicolumn{6}{|c|}{ (17) Emergency Contacts } \\
\hline \multirow{2}{*}{ Last Name } & First Name & Relationship & $(1)$ & - & \\
\hline & risst wame & Relationship & ( ) & - & Other Information \\
\hline Last Name & First Name & Relationship & Phone Number & & Other Information \\
\hline
\end{tabular}

(18. Comments

\section{Long Term Care Facility}

(A) Resident of Long Term Care Facility at time of Diagnosis: $\quad \square y_{e s} \square$ No (B) Resident of Long Term Care Facility within the last 2 Years: $\square$ Yes $\square$ No If Yes to A or B: $\square$ Nursing $\square$ Hospital $\square$ Residential $\square$ Mental

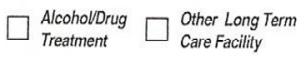

Comments
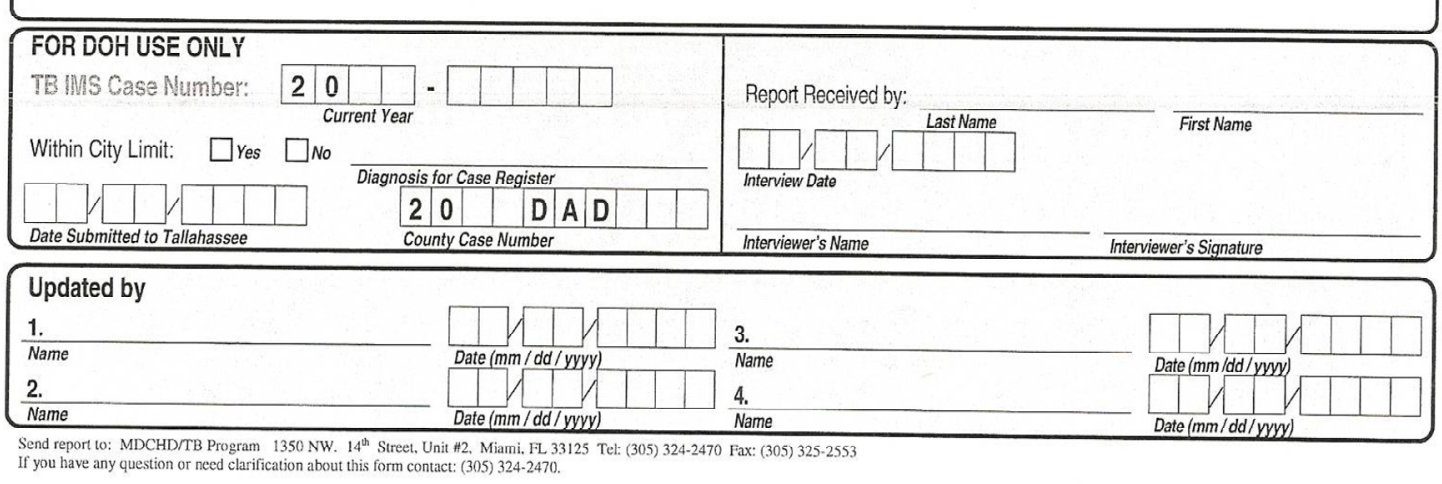

REV. 5/21/2008 3:03:00 PM REAt_..,TB REPORT 


\section{Appendix D \\ SPS REQUEST FORM}

I, Regina McDade, request to copy the Spiritual Perspective Scale (SPS) for use in my research entitled, The Influence of Spirituality on TB Medication Adherence Among African Americans and Haitians, and indicate the nature of the research (Doctoral Dissertation).

In exchange for this permission, I agree to submit to Dr. Pamela G. Reed a copy of items 1 and 2 below, and item 3 if available:

1) An abstract of my study purpose, framework, and findings, especially which includes the correlation's between the SPS scale scores and any other measures used in my study. (This will be used by Dr. Reed to assess construct validity).

2) The reliability coefficient as computed on the scale from my sample (Cronbach's alpha).

3) A computer of the SPS data and demographic data, and the data coding dictionary (to decipher coded data).

Any other information or findings that could be helpful in assessing the reliability or validity of the instrument would be greatly appreciated (e.g., problems with items, comments from subjects, other findings).

These data will be used to establish a normative database for clinical populations. No other use will be made of the data submitted. Credit will be given to me in reports of normative statistics that make use of the data I submitted for pooled analyses.

Date: May 7, 2008

Researcher's Name: Regina McDade RN, MPH

Professional Position: Tuberculosis Clinical Care Coordinator

Mailing Address: 9015 N.W. $9^{\text {th }}$ Court

Miami, FL. 33150

Email Address: regcarp1@yahoo.com

Permission is hereby granted to copy the SPS for use in the research described above.

Pamela G. Reed, RN, PhD, FAAN Date: 


\section{Regina,}

You are welcome to use the scale (attached). I ask only that you complete and return the Request Form by email, for my files. Your study sounds very interesting and significant.. I assume by mixed method you'll be collecting qualitative data as well.

The SPS works very well across various populations. Let me know if you have any questions.

Best wishes in your research plans and congratulations on advancing to doctoral candidacy!

Pam

Pamela G. Reed, PhD, RN, FAAN

Professor

College of Nursing

University of Arizona 
Appendix E

Semi-structured Interview Guide

Thank you for agreeing to participate in this study on the relationship between spirituality, knowledge of TB and TB medication adherence among African Americans and Haitians. Spirituality is a relationship with a transcendent force (state of being beyond physical limits) that brings meaning and purpose to one's existence and affects the way in which one functions in the world. The objective of this interview is to learn about your spirituality and the role of spirituality in your life after being diagnosed with $T B$.

\section{Role of Spirituality}

1. Tell me what does spirituality mean to you?

2. Do you consider yourself to be a spiritual person? If so, why?

3. Tell me how you practice spirituality in your everyday life?

4. Tell me about your spiritual practices.

\section{Medication Adherence}

5. Tell me about medication adherence.

6. Tell me how you take your medication.

7. Tell me how your family feels about you taking the medications?

\section{Knowledge of TB}

8. Tell me how you first learned bout TB?

9. Tell me what you know about TB? What is it?

10. What are your sources of information on TB?

11. What do you think I should know? 


\section{Appendix F}

\section{Letter of Support from Miami Dade County Health Department}

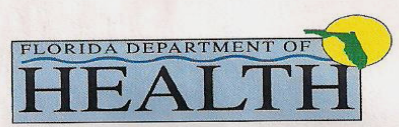

July 8, 2009

Dr. Robert Hood, Assistant Director

Statewide Research Office

4030 Esplanade Way

Floor 02, Room 280M

Tallahassee, Florida 32399-1749

Re: Letter of Support from Miami Dade County Health Department

Dear Dr. Hood:

Regina McDade RN, MPH a doctoral candidate at Florida International University College of Education proposes to conduct a study on "The Relationship Between Spirituality, Knowledge and Tuberculosis (TB) Medication Adherence among African and Haitian TB patients at Jackson Memorial Hospital and Miami-Dade County Health Department TB Unit.

To shed light on spirituality and TB medication adherence, this mixed-method study will explore the relationship between spirituality, knowledge and TB medication adherence among African Americans and Haitians. A correlational study will be conducted using TB patients' Medication Adherence Records and data from the Spiritual Perspective Scale to determine if a relationship exists between spirituality and TB medication adherence. In addition, insight from phenomenological interviews with a small subsample of TB patients will be used to further examine the relationship between spirituality, knowledge and medication adherence. This study appears to provide healthcare professionals and our community additional knowledge that is crucial for successful completion of TB medication therapy and TB control activities in our community.

The Miami-Dade County Health Department supports Regina McDade's request to conduct this study at Miami Dade County Health Department TB units and include TB patients in the TB Control and Prevention Program.

Sincerely,

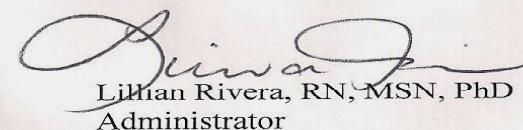

$\mathrm{LR} / \mathrm{ML} / \mathrm{gp}$

cc: Regina McDade, RN, MSN

Lillian Rivera, RN, MSN, PhD, Administrator $=$ Miami-Dade County Health Department
8323 NW 12h Street, Suite 212, Miami, Florida 33126
Tel: (786) 336-1259 Fax: (786) 336-1297
Website: www.dadehealth.org




\section{Appendix G}

\section{Florida International University Institutional Review Approval Letter}

\section{FLORIDA \\ INTERNATIONAL UNIVERSITY}

Office of Research Integrity Research Compliance, MARC 430

\section{MEMORANDUM}

To:

Regina McDade

CC:

Dr. Tonette Rocco

From:

File

Date:

Chris Grayson, CIM, Associate Director of Research Compliance 15

December 15, 2009

Proposal Title: "The Relationship Between Spirituality, Knowledge and Tuberculosis Medication Adherence Among African Americans and Haitians"

Approval \# 120908-02

The Institutional Review Board of Florida International University has re-approved your study for the use of human subjects. Your IRB approval date is December 9, 2009 and this approval will expire on December 9,2010 . As a requirement of IRB approval you are required io:

1) Provide immediate written notification to the IRB of:

- Any additions to, or changes in the procedures involving human subjects,

- Every serious or unusual or unanticipated adverse event as well as problems with the rights or welfare of the human subjects. Confirmation of receipt of serious $A E$ reports must be made with the IRB office.

2) Utilize copies of the date stamped consent document(s) for the recruitment of subjects and receive annual renewal of consent documents.

3) Receive amnual review and re-approval prior to your expiration date

Special Conditions: N/A

Please note your approval number is indicated above. For further information, you may visit the ORI - Human Subjects website at http:/fori. fiuedu/RB.html. 


\title{
Appendix $\mathrm{H}$
}

\section{University of Miami Institutional Review Board Approval Letter}

\author{
[x] UM Logo \\ EXPEDITED - APPROVAL
}

January 20,2009

Alexis Powell , M.D.

University of Miami

Department of Infectious Diseases

Campus, Locator Code: $90 \mathrm{~A}$

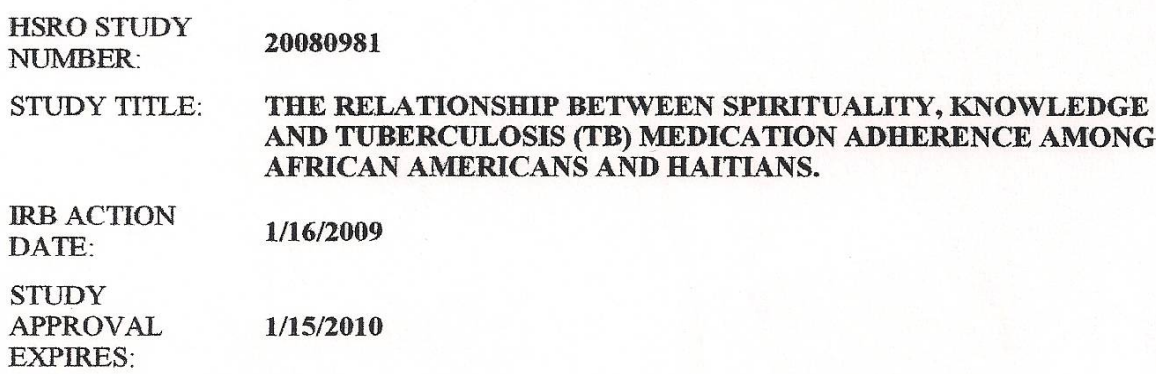

On January 16, 2009, an IRB Chair approved the following items under the expedited review process

\section{APPROVAL INCLUDES:}

New Research Protocol

HIPAA Form B - Revision Date 03/27/06 (English \& Creole Versions Only)

Research Materials (English Versions Only)

- Informed Consent Form (University of Miami \& Jackson Health Systems)

- Spirituality Perspective Scale

- Medication Administration Record

- TB Case/ Suspect Report

- Semi-Structured Interview Guide

NOTE: Translations of IRB approved study documents, including informed consent documents, into languages other than English must be submitted to HSRO for approval prior to use.

Note to PI: IRB approvals from Miami-Dade County Health Department and Florida International University are required prior to the initiation of study activities with these sites. Please submit the approvals, once they are obtained, via a notification form.

You must send a copy of the signed authorization forms to the Office of HIPAA Privacy \& Security, 
PAC 409, Locator M-879, telephone: 305-243-5000

Note: The JHS CRRC has granted permission for the study to occur at Jackson Health System. All JHS subjects must be consented with the approved and watermarked JHS Informed Consent

Please be reminded that the Jackson informed consent document can only be used at Jackson Health System.

A request to continue this study must be submitted to the HSRO at least $\mathbf{4 5}$ days before IRB approval expires. If this study does not receive continuing IRB approval prior to expiration, all research activities must be ceased, and may officially be suspended or terminated

All principal investigators must abide by and comply with all policies and procedures for the conduct of human subject research as posted on the HSRO website (http://www.hsro.miami.edu)

Sincerely,

This is a representation of an electronic record. that was signed electronically and this page is the manifestation of the electronic signature]

Amanda Coltes-Rojas, MPH, CIP

Director

Regulatory Affairs \& Educational Initiatives

/vc

cc:

IRB File

Regina McDade

Human Subjects Research Office (M809)

PO Box 016960, Miami, Florida 33101

1500 NW 12 Avenue, Suite 1002 , Miami, Florida 33136

Tel: $305-243-3195$

Fax: 305-243-332 


\section{Appendix I}

\section{Jackson Health System Clinical Research Review Committee Approval Letter}

Sent: Wednesday, November 12, 2008 3:44 PM

To: McDade, Regina

Cc: Viamonte, Kenia F; Paterson, Marjorie A; Dort, Kimberly (JMH); Del Rosario-Rivera, Yannira;

Castillo, Elena; Insignares, Kelly

Subject: epros \#20080981

Subject: CRRC Approval - Eprost 20080981

The Clinical Research Review Committee reviewed an approved the study listed below on November 12, 2008.

eProst 20080981

Study Name: What is the Relationship Between Spirituality, Knowledge and TB medication Adherence Among African American and Haitian TB Patients?

PI: McDade, Regina

Thank you for working with the Clinical Trials Office.

Eve Sakran, MS

Director

JHS Clinical Trials Office

Jackson Health System

Jackson Medical Towers

$1500 \mathrm{NW} 12^{\text {th }}$ Avenue

Miami, Florida 33136

Phone: $305-585-7226$

Fax: 305-585-6144 


\section{Appendix J}

State of Florida Institutional Review Board Approval Letter

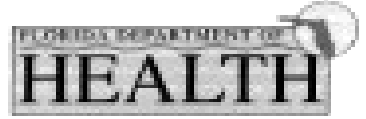

\begin{tabular}{|c|c|}
\hline $\begin{array}{l}\text { CherteCrix } \\
\text { Coverest }\end{array}$ & 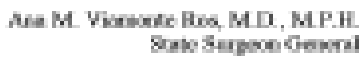 \\
\hline
\end{tabular}

NOTIFICATION OF INSTITUTIONAL REVIEW BOARD APPFONAL.

May 15,2000

To: Regina Yratte McDade

Protocol Title: THE RELATIONSHIP BETWEEN SPIRTUALTY, KNOWLEDGE ANO TUBERCULOSIS (TB) MEDICATION ADHERENCE AMONG AFRICAN AMERICANS AND HAITIANS

DOH IRE Number: HOSO24

Fundrg Agency:

Submission Type: Prosocol H09024

Review Type: Expeditad Raview

Approval Date: May 15, 2009
Expiration Date: May 14, 2010

The Deportmant of Health Insstutianal Reviow Boord, or representative, detsmined your study involves no more than minimal risk and meets the criteria for expeciled review. Is has been granted expedited approval. The study is approved for implementation.

As a reminder, the IRB must review and approve al human subjects research protocols at intervals appropriate io the degree of risk, but not less than ance por year, You are responsible for completing a continuing review application for this project at least 60 derys prier to the expiration date of Mey 14, 2010. Action is required even if your study is closing. Falure to complebe an application for continuing review at loast 60 days in advanoe of expiration is considered non-compliance by the Depertment of Heath, and may resulh in closure of the study, reporting to instibutional officisis, and reporting to federal regulstory authorities, and suapension of tunding. if funded by $\mathrm{DOH}$.

Under federal regulations, if the IR8 does not appreve an application to continue research prior to expiration, then authorization to continue research expires automafcally and all reseorch must stop. Federal regulations do not allow ary "grace" period or allow research to continue once sutherizafon expires (eorcept in limited circumstanoses)

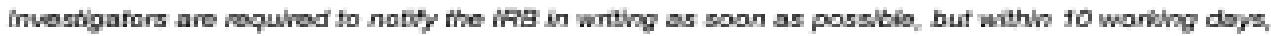
of the cceurrence of any sdverse events, unanticipoted problems, injwies, side effects, deaths, ofher problams involwing nisks to subjocts, or dowlations fom fodoral or state ragulations, or DOH poilcy.

The IRB has approved exactly what was submitted. Any revisions to this protocol or consent form, no matter how minor, must be presented to the IFB for raview and apoeaval before implementation of the charoes, extept whece necessary to eliminehe hecard to human subjects. If a change is required to 


\section{HEALTH}

Charlecrist

Aos. Y. Virtax $\operatorname{Bin}$ YD, MP.H She Sugove Geacril

eliminabe an immediate hazard, the IRB should be notifiod as soon as possible but no later than 10 wakking derys.

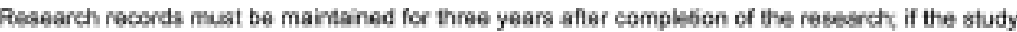
involves medics trestment, it is recommended that reconds be maintained for eight years.

If you hava any questions, or if we can be of any aseistance, please contact the Departmant of Haalen IRB at (850) 245-4595 of tol-free in Flarida (886)-433-2775.

You may also visit our wobsite at: hitp:Mpublichealthetrics net:

Thark you for your cooperation with the IRB

Sincerely.

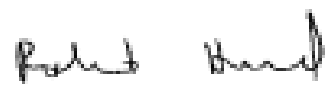

Robert Hood, Ph D

Ethics and Human Research Protection Program

Assistant Director, Office of Public Health Resoarch

Fedaral Wide AssuranceN: 0000-4682

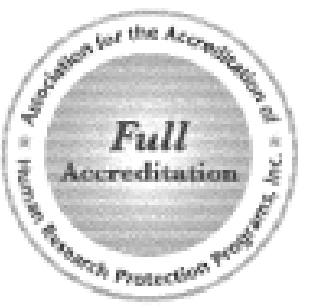

Wifee of the Sake Sapon thanal

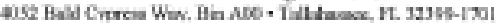




\section{VITA}

\section{REGINA Y. MCDADE}

March 13, 1964

1986

1992

1985-1986

1986-1988

1988-1990

1990-1993

2001-2008

1993
Born, Miami, Florida

Bachelor of Science in Nursing Howard University, Washington, D.C

Master of Public Health

Florida International University, Miami, Florida

Senior Health Assistant

Howard University Hospital, Washington, D.C

Clinical Nurse

Howard University Hospital, Washington, D.C.

Home Health Nurse/Hospital Coordinator

Visiting Nurse Association, Miami, Florida

Senior Community Health Nurse

Miami-Dade County Department of Health, Miami,Florida

Adjunct Instructor College of Health and Urban Affairs, School of Health, Department of Public Health Florida International University

Clinical Care Coordinator Office of Tuberculosis Control Jackson Health System, Miami, Florida

\section{PUBLICATIONS AND PRESENTATIONS}

Carpenter, R. (1994). (November, 1994). Tuberculosis Case Management: the Effect on Hospital Length of Stay and Cost. Paper presented at the National Case Management/ Critical Path Systems Conference, Atlanta, Georgia.

Carpenter, R. (1997). Tuberculosis Case Management. Paper presented at the American Lung Association Professional Seminar, Davie, Florida.

Carpenter, R. (1997). Tuberculosis case management: the effect on hospital length of stay and cost. Case Manager Journal, July/August. 
McDade, R. (2007). A Mixed Method Study of Spiritualtiy and the Influence on TB Medication Adherence. Paper presented at the Florida International University College of Education Research Conference, Miami, Florida.

McDade, R. (2008) A Mixed Method Study of the Relationship Between Spirituality, Knowledge and TB Medication Adherence Among African Americans and Haitians. Paper presented at the American Education Research Conference Preconference, St. Louis, Missouri.

McDade, R. (2010) Touchy Subject: Dealing with Sensitive Populations in Research. Paper presented at the Florida International University College of Education Research Conference, Miami, Florida. 